Copyright

by

Xiaohui Gao

2012 
The Dissertation Committee for Xiaohui Gao

certifies that this is the approved version of the following dissertation:

\section{Characterization of Cluster/Monomer Ratio in Pulsed Supersonic Gas Jets}

Committee:

Michael C. Downer, Supervisor

Michael F. Becker

Boris N. Breizman

Todd Ditmire

John W. Keto 


\title{
Characterization of Cluster/Monomer Ratio in Pulsed Supersonic Gas Jets
}

\author{
by \\ Xiaohui Gao, B.S.
}

\author{
DISSERTATION \\ Presented to the Faculty of the Graduate School of \\ The University of Texas at Austin \\ in Partial Fulfillment \\ of the Requirements \\ for the Degree of \\ DOCTOR OF PHILOSOPHY
}

THE UNIVERSITY OF TEXAS AT AUSTIN

December 2012 
To my parents and sister. 


\section{Acknowledgments}

I would like to express my deep appreciation and gratitude to my advi-

sor, Dr. Michael Downer, for his continuous support throughout my graduate life. This dissertation would not have been possible without his patient guidance and constant encouragement. I would also like to offer my sincerest thanks to my committee members, Dr. Michael Becker, Dr. Boris Breizman, Dr. Todd Ditmire, and Dr. John Keto, for their insightful advice and comments.

I am very thankful to Dr. Bonggu Shim and Dr. Xiaoming Wang, who taught me lab skills and worked together; to Dr. Alex Arefiev, for his help with many theoretical and numerical aspects of this work. I would like to thank all the past and present members of the high intensity lab, Dr. Rafal Zgadzaj, Dr. Peng Dong, Zhengyan Li, Dr. Watson Henderson, Hai-En Tsai, Dr. Chih-Hao Pai, James Sanders, Rick Korzekwa, and many others, for the team efforts to keep the laser system running smoothly; I would also like to thank the surface group members, Dr. Junwei Wei, Dr. Ming Lei, Dr. Yongqiang An, Dr. Robert Ehlert, Loucas Loumakos, Aaron Roberts, and many others, for the discussion and help. I'm grateful to the staff of UT physics machine shop, cryogenics shop, and electronics shop for fabricating parts used in experiments; to James Halligan and Cathy Rapinett for taking care of various paperwork. 
I would like to thank my friends in Austin, especially Man-Fung Cheung, Wang Ge, Jianyong Mo, Ningyu Shi, Kun Yuan, Jun Zhang, and Qiu Zhao, who shared happiness and helped me weather the stress and frustration during my $\mathrm{PhD}$ life.

Finally, I owe much to my parents, Fangjun Gao and Yadi Gong, and my sister, Yangbo Gao, for their endless love and unconditional support throughout my life, especially in the past seven years when I was studying overseas.

Xiaohui GaO

The University of Texas at Austin

December 2012 


\title{
Characterization of Cluster/Monomer Ratio in Pulsed Supersonic Gas Jets
}

\author{
Publication No. \\ Xiaohui Gao, Ph.D. \\ The University of Texas at Austin, 2012
}

Supervisor: Michael C. Downer

Cluster mass fraction is an elusive quantity to measure, calculate or estimate accurately for pulsed supersonic gas jets typical of intense laser experiments. The optimization of this parameter is critical for transient phasematched harmonic generation in an ionized cluster jet at high laser intensity. We present an in-depth study of a rapid, noninvasive, single-shot optical method of determining cluster mass fraction $f_{c}(\boldsymbol{r}, t)$ at specified positions $\boldsymbol{r}$ within, and at time $t$ after opening the valve of, a high-pressure pulsed supersonic gas jet. $\mathrm{A} \sim 2 \mathrm{~mJ}$ fs pump pulse ionizes the monomers, causing an immediate drop in the jet's refractive index $n_{\text {jet }}$ proportional to monomer density, while simultaneously initiating hydrodynamic expansion of the clusters. The latter leads to a second drop in $n_{\text {jet }}$ that is proportional to cluster density and is delayed by $\sim 1$ ps. A temporally stretched probe pulse measures the 2-step index evolution in a single shot by frequency domain holography, enabling recovery of $f_{c}$. We present the theory behind recovery of $f_{c}$ in detail. 
We also present extensive measurements of spatio-temporal profiles $f_{c}(\boldsymbol{r}, t)$ of cluster mass fraction in a high-pressure supersonic argon jet for various values of backing pressure $P$, and reservoir temperature $T$.

viii 


\section{Table of Contents}

Acknowledgments $\quad$ v

Abstract vii

List of Tables $\quad$ xi

List of Figures $\quad$ xii

Chapter 1. Introduction 1

1.1 Overview of cluster research . . . . . . . . . . . . . . 1

1.2 High-order harmonic generation from various sources . . . . . 2

1.3 Phase-matching and dispersion in clustered plasmas . . . . . . 7

Chapter 2. Simulation of laser-cluster interaction 10

2.1 Overview of laser-cluster simulation . . . . . . . . . . . . . . . 10

2.2 Optical field ionization of atoms . . . . . . . . . . . . . 12

2.3 0D ionization/heating model . . . . . . . . . . . . . 13

2.4 1D isothermal expansion model . . . . . . . . . . . 25

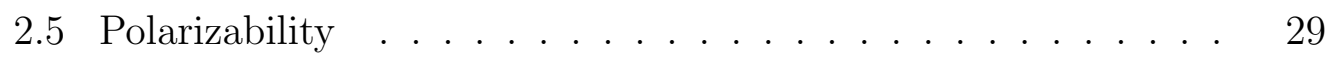

$\begin{array}{lll}\text { Chapter 3. } & \text { Properties of a cluster jet } & 31\end{array}$

3.1 Clusters in gas jets . . . . . . . . . . . . . . . . . 31

3.2 Atomic density measurement . . . . . . . . . . . . . . . . 34

3.3 Rayleigh scattering measurement . . . . . . . . . . . . 38

Chapter 4. Measurement of cluster mass fraction 41

4.1 Introduction . . . . . . . . . . . . . . . . . . 41

4.2 Method: fs-time-resolved refractive index . . . . . . . . . . . . 43

4.3 Experiment: frequency domain interferometry . . . . . . . . 45 
4.4 Experiment: frequency domain holography . . . . . . . . . . 49

4.4.1 Experimental setup and alignment procedure . . . . . 49

4.4 .2 Phase reconstruction . . . . . . . . . . . . . 54

4.4.3 Example of $f_{c}$ evaluation $\ldots \ldots \ldots \ldots \ldots$

4.4 .4 Parameter Scans . . . . . . . . . . . . . . . 60

4.4 .5 Discussion . . . . . . . . . . . . . . 66

4.5 Cluster-size distribution . . . . . . . . . . . . 71

$\begin{array}{lll}\text { Chapter 5. Harmonic generation in cluster jets } & \mathbf{7 4}\end{array}$

5.1 Introduction . . . . . . . . . . . . . . . 74

5.2 Experimental setup . . . . . . . . . . . . 75

5.3 Enhanced third-harmonic generation in cluster jets . . . . . . 77

$\begin{array}{lll}\text { Chapter 6. } & \text { Conclusions and outlook } & 82\end{array}$

$\begin{array}{ll}\text { Appendices } & 85\end{array}$

Appendix A. Pump-probe absorption measurements $\quad 86$

Appendix B. C++ code for ionization/heating of the cluster 88

$\begin{array}{ll}\text { Bibliography } & 100\end{array}$ 


\section{List of Tables}

2.1 Comparison of $Z_{c}$ in a PIC simulation to $Z_{c}$ computed by our model ......................... 24

4.1 Cluster mass fraction measured by various methods. . . . . . . 42 


\section{List of Figures}

1.1 Applications of intense laser interaction with clusters. . . . . . 2

1.2 High-order harmonic generation from various sources . . . . . 3

1.3 Single argon atom response in an $800 \mathrm{~nm}, 40 \mathrm{fs}, 2 \times 10^{14} \mathrm{~W} / \mathrm{cm}^{2}$ laser pulse calculated by the Lewenstein model . . . . . . . . . 4

1.4 Recent progresses in improving the conversion efficiency. . . . 7

2.1 False color map of charge state of an argon atom vs. peakintensity and pulse duration (FWHM) of an $800 \mathrm{~nm}$ pulse. . .

2.2 Computed charge state vs. initial cluster atomic density for a cluster irradiated by a $40 \mathrm{fs}, 800 \mathrm{~nm}, 4 \times 10^{14} \mathrm{~W} / \mathrm{cm}^{2}$ pulse. .

2.3 Time evolution of $Z_{c}$ and $T_{e}$ with various modifications for a cluster exposed to a $40 \mathrm{fs}, 800 \mathrm{~nm}$ pulse with peak intensity of

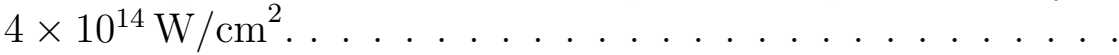
3 4 7

Time evolution of fractional population of argon ions in a cluster irradiated by a $40 \mathrm{fs}, 800 \mathrm{~nm}$ pulse with peak intensity of $4 \times$ $10^{14} \mathrm{~W} / \mathrm{cm}^{2}$. . . . . . . . . . . . . . . .

2.5 False color map of $Z_{c}$ and $T_{e}$ in an argon cluster versus peakintensity and pulse duration of an $800 \mathrm{~nm}$ pulse. . . . . . . . .

2.6 Charge state of Ar cluster (red) and monomer (black) at $t=$ $50 \mathrm{fs}$ for an $800 \mathrm{~nm}, 40 \mathrm{fs}$ pump pulse vs. pump intensity. . . .

2.7 (a) Electric field inside the cluster (b) Rates of ADK ionization, collisional ionization, and laser-assisted ionization. . . . . . . .

2.8 Snapshots of electron density profile and charge state profile during the expansion for a cluster of initial radius of $10 \mathrm{~nm}$. .

2.9 Evolution of $Z_{c}$ during the expansion for a cluster of $7 \mathrm{~nm}$ radius subject to a $40 \mathrm{fs}, 800 \mathrm{~nm}$ pulse with two different peak intensities. 27

2.10 (a) Evolution of electron temperature during an isothermal expansion. (b) Charge state distribution . . . . . . . . . 28

2.11 The real and imaginary part of the polarizability. . . . . . . . 30

3.1 (a) Image of the gas jet. (b) Schematic of the solenoid valve and the home-made nozzle. . . . . . . . . . . . 
3.2 Schematic setup for gas atomic density measurement . . . . .

3.3 (Left) Sample interferogram at $500 \mathrm{psi}, 0.8 \mathrm{~ms}$ after the valve opens. $z=0$ is the edge of the gas jet. (Right) Extracted phase

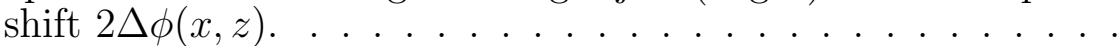

3.4 (Left) Single-pass phase shift $\Delta \phi(x)$ at $z=1 \mathrm{~mm}$. (Right) Gas density obtained after FFT-based Abel inversion for noisy data and fitted curve with two different $k_{\max } . \ldots . . . . .37$

3.5 Sub-ms time-dependent phase shift of a neutral gas jet . . . . 38

3.6 Rayleigh scatter vs. time after the valve opens . . . . . . . . . 39

3.7 Rayleigh scatter vs. backing pressure . . . . . . . . . . . . . . 40

3.8 (a) Fluorescence and Rayleigh scatter. (b) Fluorescent spectra from laser-produced plasma. . . . . . . . . . . . . . . . . . 40

4.1 Schematic set-up for fs-time-resolved measurement of $\mathrm{n}_{\text {jet }}(\tau)$. . 46

4.2 Fs-time-resolved phase shifts measured by FDI. . . . . . . . . 47

4.3 Multi-shot averaged time-resolved phase shifts measured by FDI. 48

4.4 Schematic set-up for single-shot, fs-time-resolved measurement of cluster gas jet refractive index $n_{\text {jet }}(\tau) . . . . . . . .$.

4.5 Image of the mesh used to determine the object plane and to calibrate the magnification. . . . . . . . . . 51

4.6 Image when the probe is behind the pump pulse. . . . . . . . 52

4.7 Calibration of the chirp parameter. . . . . . . . . . . . 55

4.8 Spectral amplitude and phase extracted using Fourier transform method. . . . . . . . . . . . . 55

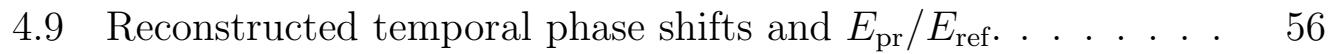

4.10 Two fs-time-resolved probe phase shifts $\Delta \phi_{\mathrm{pr}}(\tau)$, each measured in a single shot, at time $t=0.3 \mathrm{~ms}$ (black) or $0.8 \mathrm{~ms}$ (red) after opening of gas jet valve. . . . . . . . . . . . 57

4.11 Phase shift vs. energy delivered to the gas jet. . . . . . . . . . 58

4.12 Error of $f_{c}$ caused by error in $I_{\mathrm{pu}}$. . . . . . . . . . . . . . . . 59

4.13 Cluster fraction $f_{c}$ vs. time $t$ after opening of valve of gas jet. Insert: Phase shift from the neutral gas jet vs. $t . \ldots 60$

4.14 Rayleigh scatter and $f_{c} N_{\text {tot }}$ vs. time $t$. . . . . . . . . . . 61

4.15 (a) Phase shifts at various position. (b) Cluster fraction $f_{c}$ vs. transverse position. . . . . . . . . . . . 62 
4.16 (a) Cluster fraction $f_{c}$ vs. position for several different pressures.(b) Cluster fraction $f_{c}$ vs. pressure. . . . . . . . . .

4.17 (a) Measured phase shifts at various temperature.(b) Cluster fraction $f_{c}$ vs. temperature. . . . . . . . . . . 66

4.18 Perspective view of the time-resolved phase shifts. . . . . . . . 67

4.19 Measured and calculated phase shifts vs. position. . . . . . . . 68

4.20 Measured phase shifts (red squares) and simulated cluster contribution to the refractive index (blue line) . . . . . . . . .

4.21 Measured (open circles) and calculated (solid line) absorption $A(\tau)$ as a function of the delay $\tau \ldots . . . . . . . .72$

5.1 Setup for harmonic detection . . . . . . . . . . . . . . 75

5.2 Third harmonic spectrum (1 shot) . . . . . . . . . . 76

5.3 Fifth harmonic spectrum $\left(10^{4}\right.$ shots $)$. . . . . . . . . . . 77

5.4 Autocorrelation of pump and probe to check zero delay. The result is shifted by -15 fs to have it center at time zero. . . . . 78

5.5 (a) THG signal. For each data point, the CCD was exposed for 100 shots (b) Contribution from the strong pulse. . . . . . . . 79

5.6 Third harmonic spectra at three different delays. The CCD was exposed for 100 shots. . . . . . . . . . . . 79

5.7 Non-collinear pump-probe schematic. . . . . . . . . . . . 80

6.1 The refractive index (blue) and absorption coefficient (red) for a argon jet of $N_{\text {tot }}=10^{18} \mathrm{~cm}^{-3}, f_{c}=0.8, r_{c}=20 \mathrm{~nm} . \quad \ldots \quad 83$

A.1 Setup for absorption measurement. . . . . . . . . . . . . . 86

A.2 Absorption measurement . . . . . . . . . . . . . . 87 


\section{Chapter 1}

\section{Introduction}

\subsection{Overview of cluster research}

Research in Van-der-Waals bonded clusters formed in gas jets dates back to 1956 [1], when supersonic gas jets became popular as intense atomic and molecular beam sources. As they bridge the gap between atoms and bulk materials, clusters exhibit many fascinating features different from atoms and solids and permit the study of the transition from atomic to bulk properties. Cluster's structure [2], the influence of jet parameters on the cluster size [3] and many other properties of clusters have been extensively studied since the 1970s. A remarkable renaissance has been seen since the 1990s owing to the advancement of ultrashort high power laser technology. Studies of laser-cluster interaction is of fundamental interest and of practical importance. Interaction of intense laser pulses with atomic clusters has impacted several areas of laserplasma science [4] (see Fig. 1.1). It has opened up applications for table-top

neutron sources [5], electron and ion accelerators [6-8], plasma waveguides [9], and coherent and incoherent X-ray sources $[10,11]$. The work of this dissertation is motivated by the potential of utilizing high-order harmonic generation (HHG) in a cluster jet as an efficient EUV source. In the next sections, we'll 
review HHG and discuss advantages of harmonic generation in clustered plasmas.

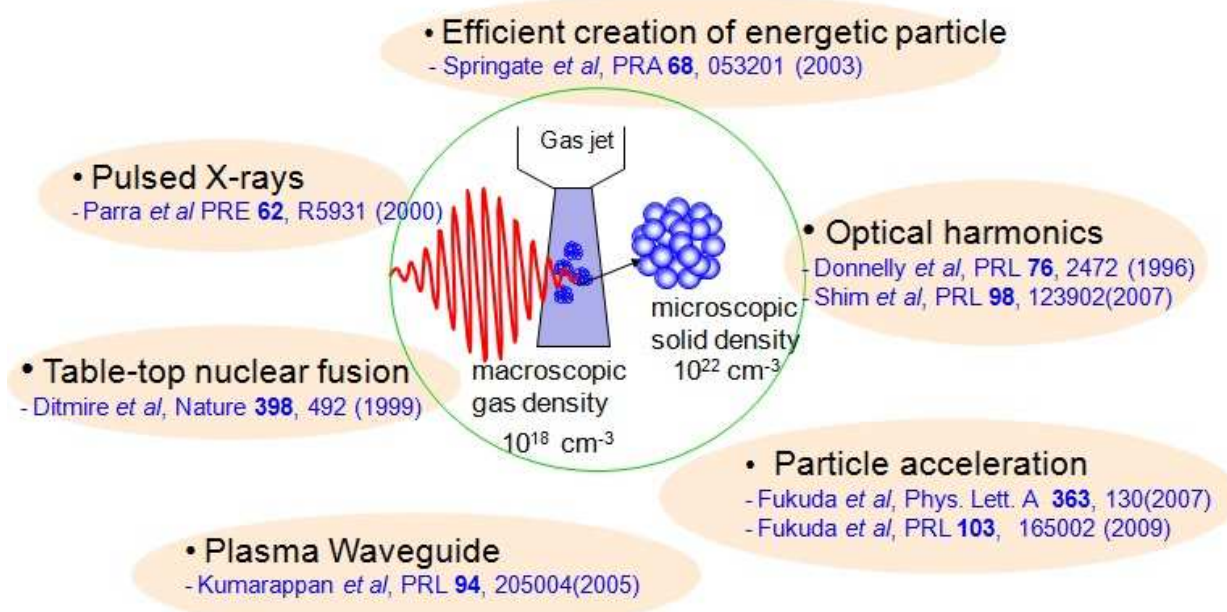

Figure 1.1: Applications of intense laser interaction with clusters.

\subsection{High-order harmonic generation from various sources}

As shown in Fig. 1.2, high-order harmonics are generated during the laser interaction with atoms, molecules, clusters, and solids at various intensities. HHG not only sheds light on laser-matter interaction, but also gives birth to attosecond science [12]. Moreover, a coherent, collimated radiation source in the extreme ultraviolet (XUV/EUV, $120 \mathrm{~nm}-10 \mathrm{~nm}$ ) and soft x-ray (10 nm$0.1 \mathrm{~nm}$ ) spectral range has wide applications ranging from lithography in the semiconductor industry to bio-imaging at the water window $(4.37 \mathrm{~nm}-2.30 \mathrm{~nm})$ in life science research. The compactness, low cost, and wide tunability makes HHG an attractive alternative to synchrotron radiation or free electron lasers. 
The low energy of high-order harmonic pulses from both gas and solid targets, however, limits their utility and continues to spur research into methods of improving HHG conversion efficiency.

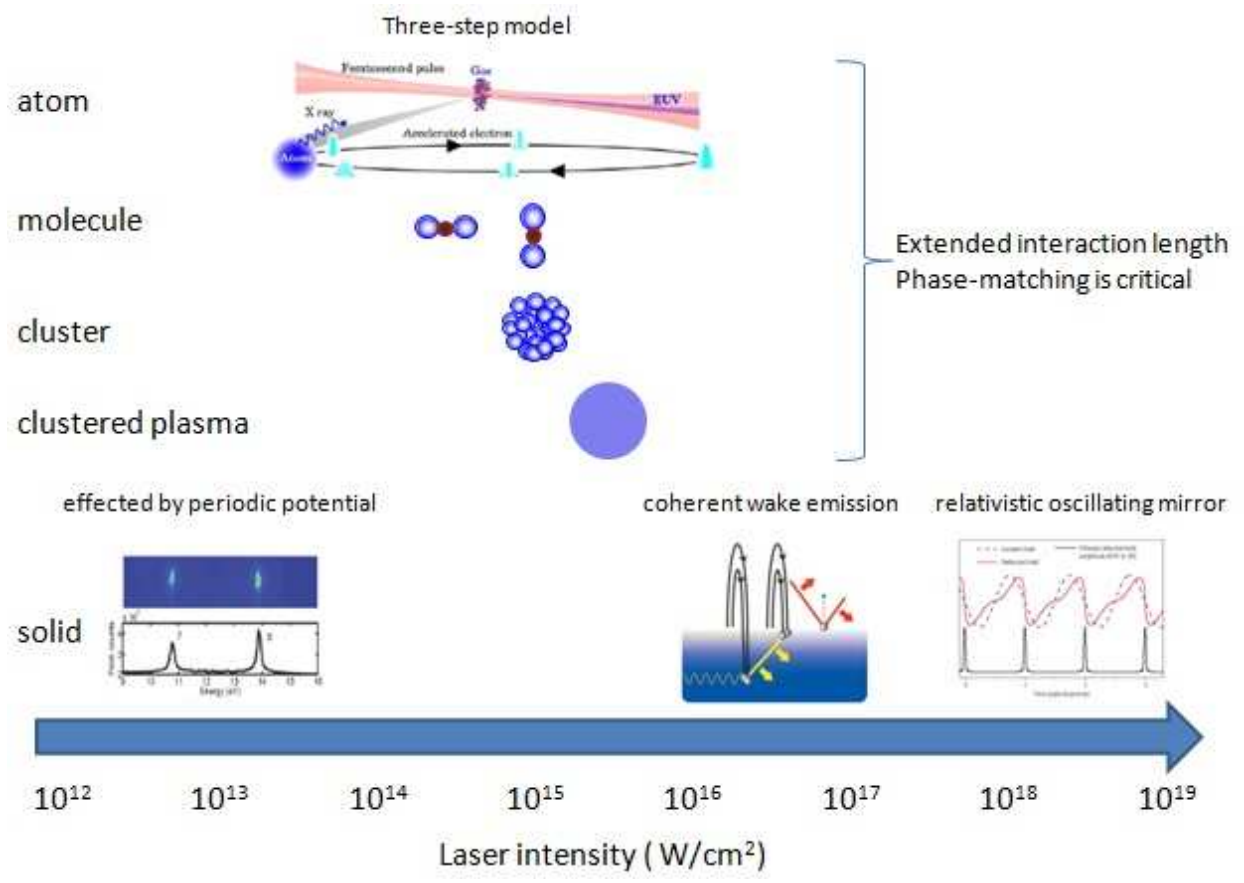

Figure 1.2: High-order harmonic generation from various sources. Parts of the figure adapted from [13-16].

A typical harmonic spectrum from atom contains three distinctive regions: an initial steep drop, a plateau and a cutoff. The cutoff energy is [17]

$$
h \nu_{\text {cutoff }}=I_{p}+3.17 U_{p},
$$

where $I_{p}$ is the ionization potential of the atom and $U_{p}$ is the ponderomotive potential. Harmonics in the region of initial drop are contributed by bound electrons through perturbative harmonic generation. The plateau and cut-off 
are interpreted semi-classically by a three-step model $[18,19]$. In this model, the production of harmonics involves three steps: ionization, propagation, and recombination. First, the electron is set free from the atom by tunnel ionization in the strong laser field; second, the free electron propagates in the oscillating laser field and acquires kinetic energy; third, the returning electron may recombine with the parent ion, releasing excess energy as a high energy photon. Fig 1.3 shows a calculated spectrum with a plateau and a cutoff using a modified Lewenstein model $^{1}[21]$. Since the contribution from bound electrons is not included, the yield of low-order harmonics is low.

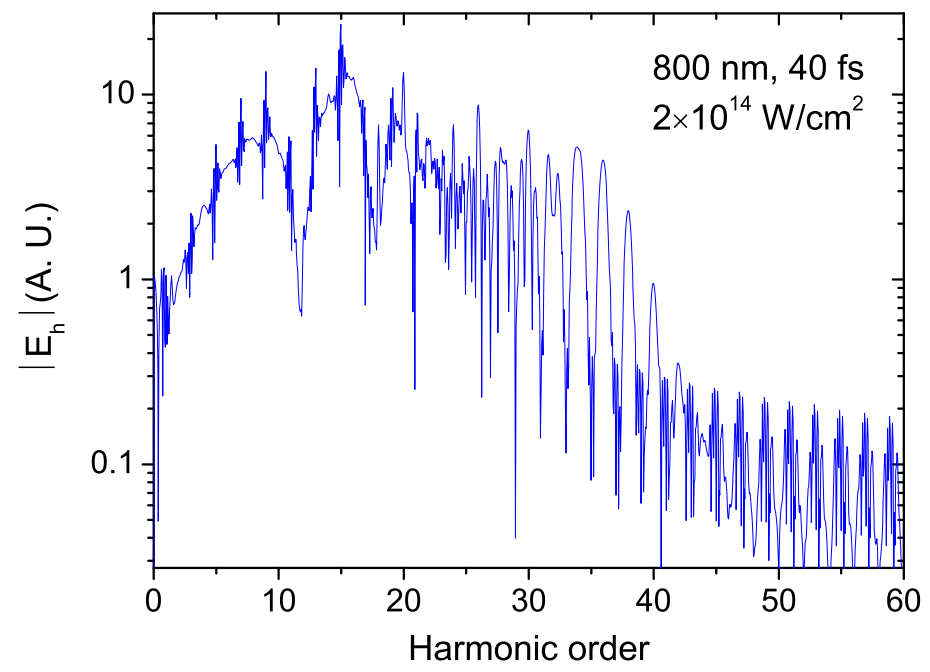

Figure 1.3: Single argon atom response in an $800 \mathrm{~nm}, 40 \mathrm{fs}, 2 \times 10^{14} \mathrm{~W} / \mathrm{cm}^{2}$ laser pulse calculated by the Lewenstein model. Eq. 1.1 gives a cutoff order of 34 .

\footnotetext{
${ }^{1}$ The calculation was based on a Matlab code in Ref. [20].
} 
One may expect HHG from ions is favorable since higher $I_{p}$ and $U_{p}$ can be used. The substantial presence of free electrons, however, results in a very limited coherence length. There are situations where a single harmonic is efficiently generated owing to a resonance enhancement ( $>100$ compared with neighbor harmonics) in a weakly-ionized metal plasma plume [22]. This enhancement is interpreted by a modified three-step model involving an autoionizing state [23].

Several interesting phenomena arise in HHG from molecules. An electron emitted at one center may recombine with the other center [24]. The harmonic yield depends on the orientation of the molecule with respect to the electric field [25]. Hence, HHG from molecules is often used as a diagnostic rather than a light source.

Harmonics from atomic clusters, first studied in 1996 by Donnelly et al. [10], show a higher cut-off order and less saturation than from monomers. This is explained in the framework of the three-step model with a modified laser field inside clusters. Vozzi et al. found that HHG from clusters are mainly contributed by the atoms on their surfaces [26]. Intuitively, one may suspect that an atom in a cluster has a greater cross section for recombination than a monomer since the returning electron may recombine to a neighbor ion. Nevertheless, a simulation result [27] shows that the contribution of recombination to neighbor ions is incoherent.

Once a cluster becomes a clustered plasma, the collective dynamics dominates. In a model proposed by Fomyts'kyi et al. [28], considerable third 
harmonic is generated because of the nonlinear oscillations of a cold electron core in a nonuniform ion background driven by the strong laser field. Shim et al. [29] observed this resonantly enhanced third harmonic generation from expanding clusters. Molecular dynamic (MD) and particle-in-cell (PIC) simulations $[30,31]$ confirm the resonant enhancement of low-order harmonics, but show that high-order harmonics are suppressed because of the dynamical stochasticity of the electron motion caused by the nonlinear resonance.

The leading edge of the laser turns solids into overdense plasmas. The collective electron dynamics at plasma boundaries generates harmonics. Due to broken symmetry, both even- and odd-order high harmonics are generated. The interaction length is limited to approximately the skin depth $c / \omega_{p}$, which is usually smaller than the coherence length. When the intensity is below the relativistic intensity, HHG is interpreted by coherent wake emission [32]. In this scenario, the Brunel electron bunches travel across a density gradient and create wakefields that emit harmonics. The harmonics have a cutoff of $\sqrt{n_{\max } / n_{\text {crit }}}$, where $n_{\max }$ is the maximum plasma density and $n_{\text {crit }}$ is the critical plasma density. At relativistic intensities, the interpretation is provided by an oscillating mirror model [33]. The oscillation of the plasma surface at the critical density leads to a Doppler shift of the reflected laser light, which gives rise to harmonics in the spectral domain. Recently, non-perturbative harmonics from solid argon were also observed [14] at $I \sim 10^{13} \mathrm{~W} / \mathrm{cm}^{2}$. 


\subsection{Phase-matching and dispersion in clustered plasmas}

Great efforts have been devoted to increasing the conversion efficiency in gas targets. From the microscopic perspective, the goal is to increase the single atom yield. If the harmonic is beyond the plateau, one needs to extend the cutoff. As Eq. 1.1 suggests, this can be achieved by increasing wavelength or $I_{p}$. On the other hand, reducing the wavelength [34] or manipulating the temporal shape of the pulse [35] may increase the recombination cross section. In a macroscopic scale, three main propagation effects limiting the conversion efficiency are absorption, dephasing and defocusing. Absorption-limited HHG can be achieved for $\lambda>10 \mathrm{~nm}[36]$. At short wavelengths, the phase-mismatch is the main limitation. Some recent results are shown in Fig 1.4.

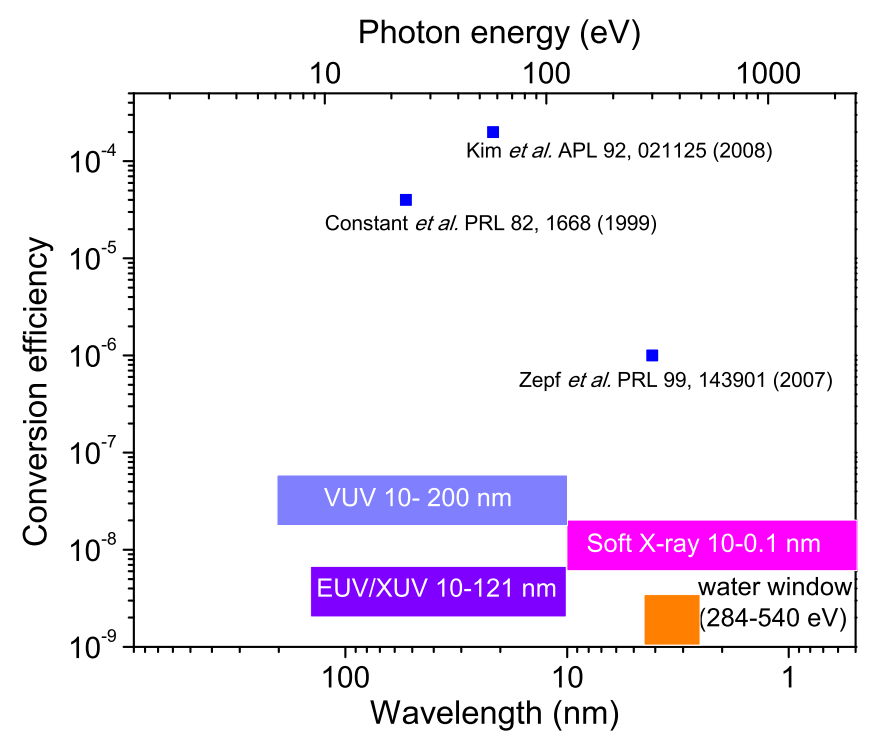

Figure 1.4: Recent progresses in improving the conversion efficiency.

To prevent the harmonic field from adding destructively, the phases of 
the driving field and harmonic field cannot differ more than $\pi$. The maximum length that harmonics can grow is called the coherence length $L_{\text {coh }}$,

$$
L_{\mathrm{coh}}=\frac{\pi}{|\Delta \mathbf{k}|}
$$

where $\Delta \mathbf{k}$ is the phase mismatch. The phase-mismatch can be written as [37]

$$
\Delta \mathbf{k}=\Delta \mathbf{k}_{\text {geo }}+\Delta \mathbf{k}_{\text {index }}-\theta \nabla I
$$

Here $\Delta \mathbf{k}_{\text {geo }}$ is the mismatch due to geometry such as Gouy phase or correction in a hollow waveguide, $\Delta \mathbf{k}_{\text {index }}$ is the mismatch due to plasma dispersion and linear atomic polarizability, $-\theta \nabla I$ is the mismatch due to the dipole phase gradient ( $I$ is the laser intensity). $\Delta \mathbf{k}_{\text {index }}=q \omega[n(q \omega)-n(\omega)] / c$ is usually the dominant term, where $n(\omega)$ and $n(q \omega)$ are the refractive indices for frequency $\omega$ and $q \omega$, respectively, and $n(q \omega) \approx 1$. Consequently, we need $n(\omega) \approx 1$. For $\lambda=0.8 \mu \mathrm{m}$, harmonics up to $50 \mathrm{eV}$ can be phase-matched in argon by tuning the pressure, but the laser intensity is limited to a level where ionization is less than 5\% [38]. Gas-filled capillary waveguides have been used effectively to suppress the destructive interference by periodically modulating of the driving laser intensity, thereby enabling quasi-phase-matched HHG in multiply-ionized argon and neon $[39,40]$. Nevertheless, the drive intensity remains limited to $I \lesssim 10^{15} \mathrm{~W} / \mathrm{cm}^{2}$ by the optical damage of the capillaries. Achieving phasematched or quasi-phase-matched HHG at high intensities is a great challenge.

Targets comprised of clusters formed by condensation in pulsed supersonic gas jets combine advantages of monomer gas targets (extended interaction length, freedom from debris, and capability of operating at high repetition 
rate) and solid targets (high local density, efficient energy absorption). Moreover, an overdense clustered plasma is small enough for the laser to penetrate, thus giving rise to a positive refractive index. This can be seen from the refractive index of uniform density clustered plasmas [41],

$$
n^{2}(k, \omega)=1-\frac{p \omega_{p}^{2}}{\omega^{2}-f \omega_{p}^{2}+i \nu \omega}
$$

where $\omega_{p}$ is the plasma frequency for the cluster density, $f$ is a geometrical factor ( $f=1 / 3$ for a sphere), $\nu$ is the collision frequency, $p=\frac{4 \pi}{3} N_{c} r^{3}, r$ is the cluster radius and $N_{c}$ is the number density of clusters. One may find $\boldsymbol{R e}\left(n^{2}\right)>1$ when $\omega<\omega_{p} / \sqrt{3}$. This positive contribution may compensate negative contribution from monomer plasma if the cluster mass fraction and internal electron density can be optimized, thus increasing $L_{\text {coh }}$ and conversion efficiency without the limit of the laser intensity [41]. Through this mechanism, clusters can enhance harmonic efficiency regardless of whether the clusters themselves or monomer ion cores are the primary source of harmonic radiation. One critical parameter is the cluster mass fraction $f_{c}$. The characterization of this parameter is the main topic of the dissertation.

In addition to $L_{\text {coh }}$, the medium length $L_{\text {med }}$ and the absorption length $L_{\text {abs }}$ also limit the output harmonic energy. Therefore, it suffices to require $L_{\text {coh }}>5 L_{\text {abs }}$ and $L_{\text {med }}>3 L_{\text {abs }}$ to achieve more than half of the maximum output [36]. 


\section{Chapter 2}

\section{Simulation of laser-cluster interaction}

\subsection{Overview of laser-cluster simulation}

A cluster is a many-body system with the number of atoms ranging from tens to millions. Simulation of laser interaction with such a system poses a great challenge. While a fully ab initio treatment is feasible for very small clusters, simulations using molecular dynamic (MD) method or rate equations are so far the only practical methods for large clusters $\left(N>10^{4}\right)[4]$. For a nanometer-sized cluster interacting with laser pulses of moderate intensity

$\left(I \sim 10^{15} \mathrm{~W} / \mathrm{cm}^{2}\right)$, macroscopical description using rate equations is a popular choice. The corresponding model first proposed by Ditmire et. al. is often called the nanoplasma model [42].

The nanoplasma model describes the ionization, heating, and expansion of a cluster after irradiation by an intense laser pulse using a set of timedependent global variables governed by coupled ordinary differential equations (ODE's). It successfully explains the main features of laser-cluster interaction (production of highly charged ion, presence of resonance). Several improvements of the nanoplasma model are made later by adopting accurate collision frequencies or ionization cross sections [43], by adding collision frequency con- 
tributed by surface-induced Landau damping [44], and by including lowering of ionization threshold [45]. The over-simplified assumption of a uniform density profile, however, causes a major discrepancy with the experiment: the absorption peak in the calculation has a much narrower time interval than in the measurement [46]. This drawback was remedied by including a radiusdependent density and temperature distribution in a one-dimensional (1D) hydrodynamic model [47]. The hydrocode gives qualitative agreement with the measured refractive index and absorption [48], although a quantitative comparison was not made. Because the hydrocode is computationally intensive, its application is limited. A modified nanoplasma model, which fits results from hydrocode by adjusting ion mass and collision frequency, was proposed [49]. The validity is solely based on hydrocode. The fitting only works for early time ( $\lesssim 500 \mathrm{fs}$ ) when the electron density is above $n_{\text {crit }}$. Moreover, the fitting is only done with limited sets of parameters. Failure with other untested parameters cannot be ruled out.

Since the expansion time for a typical cluster $(\sim \mathrm{ps})$ exceeds the duration of the pump pulse ( $\sim 40$ fs) significantly, cluster ions can be treated as cold and their expansion during the pump pulse neglected. This allows us to treat the ionization/heating and expansion of the cluster separately. In our two-stage model [50-52], the ionization/heating is treated in the framework of the nanoplasma model. The calculated fractional population of various ion species $n_{i 0}(Z)$ and electron temperature $T_{e 0}$ are then used as initial conditions for a 1D isothermal expansion model. 
In the next three sections, we'll review ionization of atoms in intense laser field, and describe in detail our zero dimensional (0D) ionization/heating model and 1D isothermal expansion model. An accurate charge state $Z_{c}$ at $\sim 1$ ps after the laser irradiation is critical for the accurate evaluation of the cluster mass fraction in Chapter 4.

\subsection{Optical field ionization of atoms}

The ionization of an atom is described by the Ammosov-Delone-Krainov (ADK) formula [53]. The formula shows excellent agreement with experiments, and therefore can be used for precise calibration of the laser intensity [54]. In atomic units, the cycle-averaged ADK rate reads

$$
\begin{aligned}
W_{\mathrm{ADK}}= & C_{n^{*} l}^{2} f(l, m) I_{p}\left(\frac{3 E}{\pi\left(2 I_{p}\right)^{3 / 2}}\right)^{1 / 2} \\
& \times\left(\frac{2\left(2 I_{p}\right)^{3 / 2}}{E}\right)^{2 n^{*}-|m|-1} \exp \left(-\frac{2\left(2 I_{p}\right)^{3 / 2}}{3 E}\right)
\end{aligned}
$$

with $C_{n^{*} l}=\left(2 e / n^{*}\right)^{n^{*}}\left(2 \pi n^{*}\right)^{-1 / 2}$, and $f(l, m)=((2 l+1)(l+|m|) !) /\left(2^{|m|}|m| !(l-\right.$ $|m|) !)$. Here the constant $e$ is Euler's number and the effective principal quan-

tum number $n^{*}=Z \sqrt{2 I_{p}}$. For sufficiently high intensities, the electron can escape classically when the external electric field overcomes the barrier created by the Coulomb potential. In this above barrier ionization (ABI) regime, the ADK formula may overestimate the ionization rate [55]. Fortunately, unless the intense pulse reaches the few-cycle limit, the leading edge of the laser pulse completely strips off electrons in the tunnel regime. The rate equations are

$$
\frac{d N_{i}}{d t}=-W_{i} N_{i}+W_{i-1} N_{i-1}, \text { for } i=0, \ldots, 18 .
$$




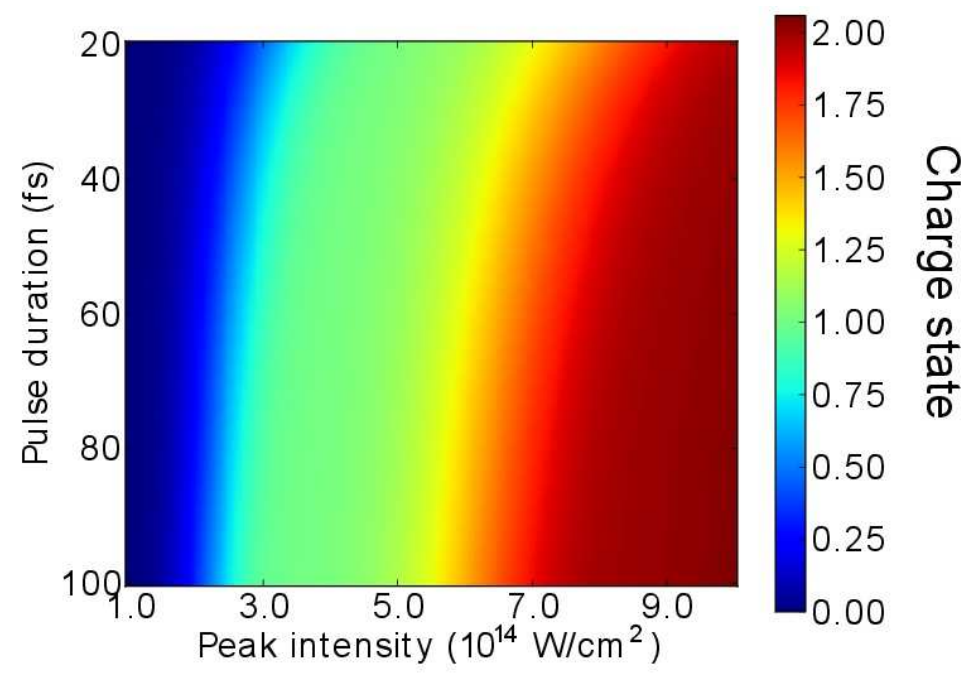

Figure 2.1: False color map of charge state of an argon atom vs. peak-intensity and pulse duration (FWHM) of an $800 \mathrm{~nm}$ pulse.

The average charge state is $\langle Z\rangle=\sum_{Z=0}^{\infty} Z N_{i}(Z) / \sum_{Z=0}^{\infty} N_{i}(Z)$.

The calculated charge state is shown in Fig. 2.1 for a range of intensities and pulse durations. For the intensity $\left(\sim 10^{15} \mathrm{~W} / \mathrm{cm}^{2}\right)$ and pulse duration $(\lesssim 100)$ fs of our interest, the monomer charge state is more sensitive to the laser intensity than to the pulse duration.

\subsection{OD ionization/heating model}

The local atomic density with a cluster $\left(>10^{22} \mathrm{~cm}^{-3}\right)$ exceeds that of monomers $\left(\lesssim 10^{19} \mathrm{~cm}^{-3}\right)$ by at least 3 orders of magnitude. This difference changes the ionization picture dramatically. In a cluster, the field that an atom feels is modified by the polarization field. In the case of $k r \ll 1$, where $k=2 \pi / \lambda$ is the wave vector of the external field and $\lambda$ is the wavelength, the 
field inside a uniform sphere of size $r$ is [56]

$$
E_{c}=\frac{3 E_{\mathrm{ext}}}{\left|\epsilon_{c}+2\right|}
$$

where $E_{\text {ext }}$ is the driving laser field. The dielectric constant is $\epsilon_{c}=1-$ $\omega_{p}^{2} /[\omega(\omega+i \nu)]$ using Drude model, where $\nu$ is the electron-ion collision frequency and $\omega_{p}=\sqrt{4 \pi e^{2} n_{e} / m_{e}}$ is the plasma frequency, $e$ and $m_{e}$ are electron charge and mass. Tunnel ionization quickly generates seed electrons. These electrons are heated, and collisional ionization takes over and dominates. If a Maxwellian electron energy distribution is assumed, the collisional (or electron impact) ionization rate coefficient $S(Z)$ for $Z \rightarrow Z+1$ is [57]

$$
\begin{aligned}
S= & 6.7 \times 10^{-7} \sum_{i=1}^{N} \frac{a_{i} q_{i}}{T_{e}^{3 / 2}}\left(\frac{1}{P_{i} / T_{e}} \int_{P_{i} / T_{e}}^{\infty} \frac{e^{-x}}{x} d x\right. \\
& \left.-\frac{b_{i} \exp c_{i}}{P_{i} / T_{e}+c_{i}} \int_{P_{i} / T_{e}+c_{i}}^{\infty} \frac{e^{-y}}{y} d y\right),
\end{aligned}
$$

where $P_{i}$ is the binding energy of electrons in the $\mathrm{i}^{\text {th }}$ shell, $q_{i}$ is the number of equivalent electrons in the $\mathrm{i}^{\text {th }}$ subshell and $a_{i}, b_{i}, c_{i}$ are constants. The collision ionization rate including continuum lowering can be written as [58]

$$
W_{\mathrm{inz}}^{\mathrm{cl}}=n_{e} S \exp \left(-\frac{\Delta \varepsilon}{k T_{e}}\right)
$$

Here $\Delta \varepsilon\left(Z, T_{e}, n_{e}\right)$ is the shift of the ionization energy, which can be approximated by the following formula [59]

$$
\Delta_{Z-1}=-\frac{\left(3\left(Z^{*}+1\right) \frac{Z \kappa e^{2}}{k_{B} T}+1\right)^{2 / 3}-1}{2\left(Z^{*}+1\right)} k_{B} T
$$


where $\kappa$ is an effective inverse screening length

$$
\kappa=\sqrt{\frac{4 \pi e^{2}}{k_{B} T}\left(Z^{*}+1\right) n_{e}}
$$

and $Z^{*}=\left\langle Z^{2}\right\rangle /\langle Z\rangle$. The rate of collisional ionization caused by the quivering electrons in the laser field can be approximated as [60]

$$
\begin{array}{r}
W_{\text {las }}=\frac{a_{i} q_{i} n_{e}}{2 \pi I_{p} \sqrt{\left(m_{e} U_{p}\right)}}\left[\left(3-\frac{I_{p}}{U_{p}}+\frac{3}{32}\left(\frac{I_{p}}{U_{p}}\right)^{2}\right)\right. \\
\left.\ln \left(\frac{1+\sqrt{1-I_{p} /\left(2 U_{p}\right)}}{1-\sqrt{1-I_{p} /\left(2 U_{p}\right)}}\right)-\left(\frac{7}{2}-\frac{3 I_{p}}{8 U_{p}}\right) \sqrt{1-I_{p} /\left(2 U_{p}\right)}\right] .
\end{array}
$$

The total rate of ionization from charge state $Z$ to charge state $Z+1$ is $W(Z)=W_{\mathrm{ADK}}+W_{\mathrm{inz}}^{\mathrm{cl}}+W_{\text {laser }}$.

Three body recombination occurs at the same time. The rate $\alpha_{3}$ is [61]

$$
\alpha_{3}=\frac{4 \pi \sqrt{2 \pi}}{9} \frac{e^{10} Z^{3}}{m_{e}^{1 / 2}\left(k T_{e}\right)^{9 / 2}} \ln \sqrt{1+Z^{2}} .
$$

The evolution of the ion density $n_{i}(Z)$ with charge state $Z$ follows the rate equation [52]:

$$
\begin{aligned}
\frac{d n_{i}(Z)}{d t} & =-\left[W(Z)+\alpha_{3}(Z) n_{e}^{2}\right] n_{i}(Z) \\
& +W(Z-1) n_{i}(Z-1)+\alpha_{3}(Z+1) n_{e}^{2} n_{i}(Z+1)
\end{aligned}
$$

The electron temperature equation is [52]

$$
\begin{aligned}
\frac{d T_{e}}{d t}= & \frac{2}{3} \frac{Q}{n_{e}}-\frac{T_{e}}{n_{e}} \frac{d n_{e}}{d t} \\
& +\frac{2}{3} \sum_{Z=0}^{\infty} \varepsilon_{i}(Z)\left[\alpha_{3}(Z+1) n_{e} n_{i}(Z+1)-S(Z) n_{i}(Z)\right]
\end{aligned}
$$


where the electron density $n_{e}=\sum_{Z=0}^{\infty} Z n_{i}(Z), Q$ is the inverse bremsstrahlung (IBS) (or collisional) absorption rate and $\varepsilon_{i}(Z)$ is the ionization potential of the corresponding ion with $\Delta \varepsilon$ included. The second term in Eq. 2.11 is the temperature decrease due to the increase in the total number of electrons. The last term is the energy loss/gain due to collisional ionization/recombination. Tunnel ionization and quivering-electron-induced collisional ionization, which draw energy from the laser field, do not change the thermal energy of electrons directly. The ionization loss of the laser energy is not considered. The IBS absorption rate for a clustered plasma is given by [58]

$$
Q=\frac{\omega_{p}^{2} \operatorname{Re} \nu(\omega)}{\left[\omega-\frac{\omega_{p}^{2}}{3 \omega}-\operatorname{Im} \nu(\omega)\right]^{2}+[\operatorname{Re} \nu(\omega)]^{2}} \frac{\left|E_{0}\right|^{2}}{8 \pi} .
$$

$\operatorname{Im} \nu(\omega)$ is often dropped. For an ideal plasma, the collision frequency is given by Spitzer's formula,

$$
\nu_{e i}=2.91 \times 10^{-6} \frac{Z n_{e}}{T_{e}^{3 / 2}} \ln \Lambda,
$$

where $\ln \Lambda$ is the Coulomb logarithm with the maximum impact parameter of $v_{e} / \omega, v_{e}=\sqrt{T_{e} / m}$ is the electron thermal velocity. The degree to which the plasma is "ideal" is usually characterized by the electron-electron coupling parameter $\Gamma_{e e}=e^{2} / k_{B} T_{e}\left(4 \pi n_{e} / 3\right)^{1 / 3}$ and degeneracy parameter $\Theta=$ $\left(3 \pi^{2} n_{e}\right)^{-2 / 3}\left(2 m_{e} k_{B} T_{e}\right) / \hbar^{2}$. A plasma is non-ideal if $\Gamma_{e e} \geq 0.1$ or $\Theta<1$. Spitzer's formula fails at tens of eVs at solid density [62]. For example, $\Gamma_{e e}=0.33$ for $Z=3, T_{e}=30 \mathrm{eV}$ and $n_{i}=2.63 \times 10^{22} \mathrm{~cm}^{3}$. We used an 
interpolation formula suited for warm dense matter $[63,64]$ :

$$
\begin{aligned}
\nu_{\mathrm{eff}}\left(T_{e}, \omega\right) \simeq & \sqrt{2 \pi} \frac{Z e^{4} n_{e}}{m_{e}^{1 / 2}\left(k_{B} T_{e}\right)^{3 / 2}} \\
& \times \ln \left(1+\frac{1.32}{\sqrt{2 \pi}} \frac{k_{B} T_{e}}{\left(m_{e}^{1 / 2} Z e^{2} \tilde{\omega}\right)^{2 / 3}}\right) F\left(T_{e}, \hbar \omega\right),
\end{aligned}
$$

where $\tilde{\omega}=\max \left(\omega, \omega_{p}\right)$ and the Fermi factor $F\left(T_{e}, \hbar \omega\right)$ is

$$
F\left(T_{e}, \hbar \omega\right)=\frac{3 \sqrt{\pi}}{4} \Theta^{3 / 2} \ln \left(\frac{1+e^{y}}{1+e^{y-z}}\right) \frac{1}{1-e^{-z}},
$$

where $y=\mu / k_{B} T$ and $z=\hbar \omega / k_{B} T, y$ was found using an interpolation formula [65]

$$
y=-\frac{3}{2} \ln \Theta+\ln \frac{4}{3 \sqrt{\pi}}+\frac{0.25054 \Theta^{-1.858}+0.072 \Theta^{-1.858 / 2}}{1+0.25054 \Theta^{-0.858}} .
$$

The electron-surface collision can be chosen as $\nu_{s}=v / r$, where $r$ is the cluster radius and $v=\sqrt{v_{e}^{2}+v_{\text {osc }}^{2}}$ [44]. We evaluated the contribution of this term for our parameters and found it not important. This contribution matters when the collision frequency is low. This may occur at the Mie resonance when $n_{e}$ sweeps downwards through $3 n_{\text {crit }}$ during a uniform expansion. Since this term introduces radius dependence, it is not included in the following calculation.

For large clusters irradiated by moderate intensities, the fraction of electrons leaving clusters is negligible [47]. The Coulomb pressure is therefore neglected. The cluster radius equation can be written as [49]

$$
\frac{d^{2} r_{c}}{d^{2} t}=5\left(\frac{P_{e}}{n_{i} m_{i}}\right) \frac{1}{r_{c}},
$$




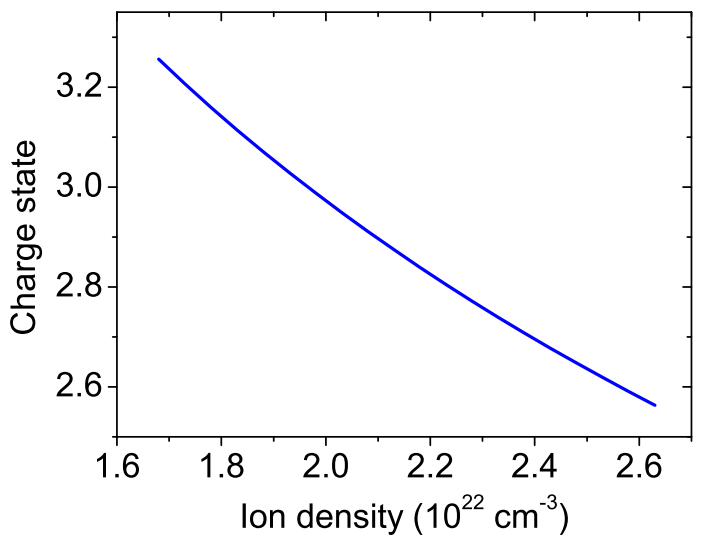

Figure 2.2: Computed charge state vs. initial cluster atomic density for a cluster irradiated by a $40 \mathrm{fs}, 800 \mathrm{~nm}, 4 \times 10^{14} \mathrm{~W} / \mathrm{cm}^{2}$ pulse.

where $P_{e}=n_{e} k T_{e}$ is the electron pressure. We assume that ions are immobile during the pump pulse. Hence, $d^{2} r_{c} / d^{2} t=0$.

The systems of ODEs were solved numerically using a Runge-Kutta solver in GNU Scientific Library (GSL). The initial conditions are the cluster atomic density $n_{i 0}$ and initial electron temperature $T_{e 0}$. We find $n_{i 0}=2.63 \times$ $10^{22} \mathrm{~cm}^{-3}$ from the lattice constant of argon clusters in a supersonic gas jet $\left(a_{\mathrm{Ar}}=0.534 \pm 0.001 \mathrm{~nm} \text { with fcc structure }[2,66]\right)^{1}$. The strong dependence of the heating/ionization on $n_{i 0}$ shown in Fig. 2.2 is not surprising because a plasma density near $3 n_{\text {crit }}\left(5.1 \times 10^{21} \mathrm{~cm}^{-3}\right)$ leads to a strong absorption. On the other hand, $T_{e 0}$ is irrelevant to the result because the initial electron density is zero. This is verified in our calculation. For numerical stability, we assume

\footnotetext{
${ }^{1}$ For $10^{3} \lesssim\langle N\rangle \lesssim 10^{5}$, fcc, hcp and random close-packed structures are mixed in argon clusters [67]. The packing fraction for fcc, hcp, and random close-packed structure are 0.74 , $0.74,0.64$, respectively. Therefore, the local atomic density may be smaller.
} 

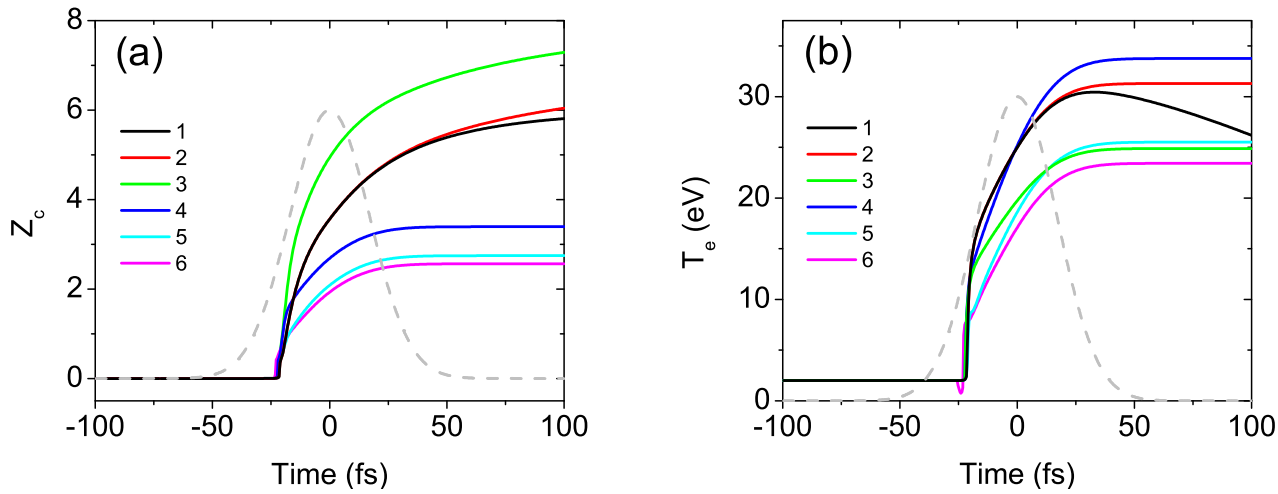

Figure 2.3: Time evolution of $Z_{c}$ and $T_{e}$ with various modifications for a cluster exposed to a $40 \mathrm{fs}, 800 \mathrm{~nm}$ pulse with peak intensity of $4 \times 10^{14} \mathrm{~W} / \mathrm{cm}^{2}$. 1 : nanoplasma model. 2: no expansion. 3: lowering of ionization potential. 4: recombination. 5: change of $T_{e}$ due to change of $n_{e}$. 6: energy loss/gain due to recombination and collisional ionization. The grey dashed line shows the intensity envelope.

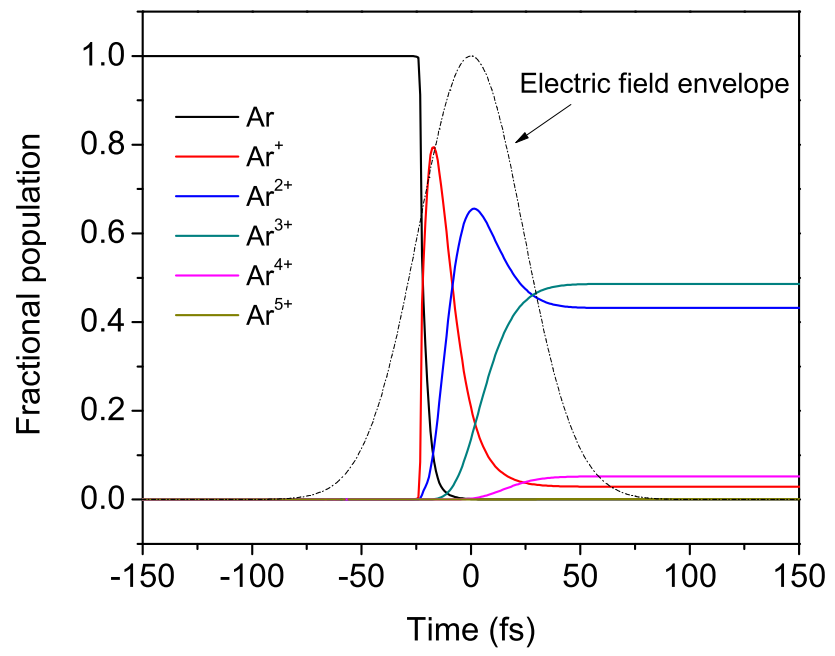

Figure 2.4: Time evolution of fractional population of argon ions in a cluster irradiated by a $40 \mathrm{fs}, 800 \mathrm{~nm}$ pulse with peak intensity of $4 \times 10^{14} \mathrm{~W} / \mathrm{cm}^{2}$. 

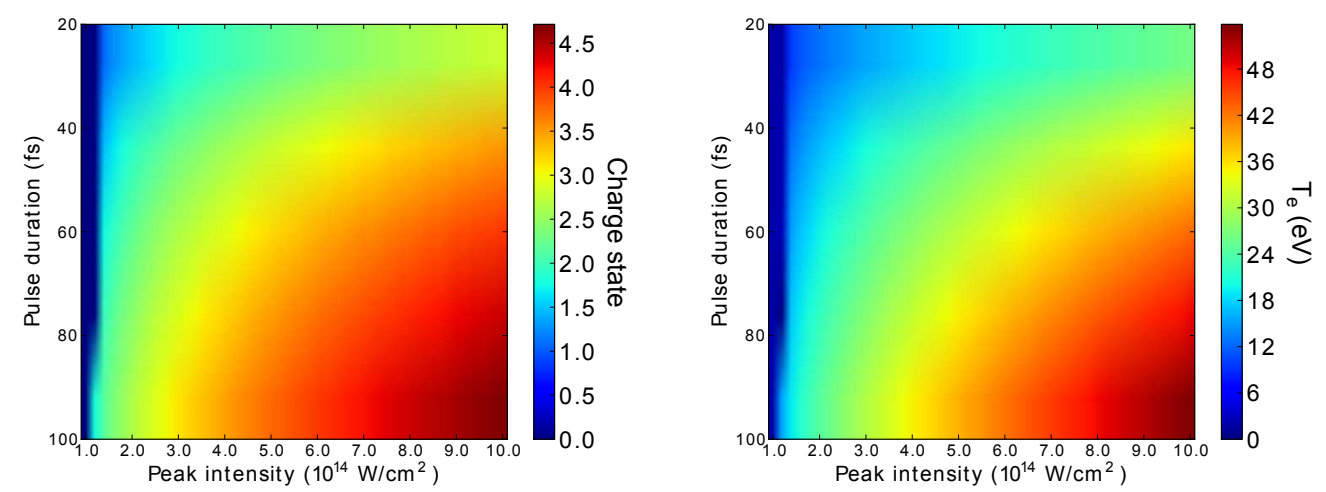

Figure 2.5: False color map of $Z_{c}$ (left panel) and $T_{e}$ (right panel) in an argon cluster at $t=1.2 \tau$ for an $800 \mathrm{~nm}$ pulse vs. pump intensity and pulse duration $\tau$. For $t \gtrsim 1.2 \tau$, the clustered plasma is in an equilibrium state in our model.

$T_{e 0}=2 \mathrm{eV}$. Evolution of $Z_{c}$ and $T_{e}$ with various modifications are plotted in Fig. 2.3. The expansion does not affect $Z_{c}$ and $T_{e}$ for $t<30 \mathrm{fs}$. This justifies our assumption that expansion is negligible during the ionization/heating. Continuum lowering of the ionization potential increases $Z_{c}$ but lowers $T_{e}$. Once three-body recombination is included, it quickly balances the collisional ionization, leaving a constant $Z_{c}$ after the pulse is gone. The $2^{\text {nd }}$ and $3^{\text {rd }}$ terms in Eq. 2.11 lower $Z_{c}$ further. For $I_{\mathrm{pu}}=4 \times 10^{14} \mathrm{~W} / \mathrm{cm}^{2}, 40 \mathrm{fs}$, we find $Z_{c}=2.56$ and $T_{e}=23.4 \mathrm{eV}$. The time evolution of various ion population is shown in Fig. 2.4. Maps of $Z_{c}$ and $T_{e}$ vs. peak laser intensity and pulse duration $^{2}$ are illustrated in Fig. 2.5. Compared with Fig. 2.1 where tunnel ionization is the only contribution, the dominance of collisional ionization leads to a smooth growth of $Z_{c}$ and $T_{e}$ except for the beginning, where $I_{\mathrm{pu}}$ is low.

\footnotetext{
${ }^{2}$ We calculated $Z_{c}$ and $T_{e}$ for $\tau=20,40,60,80,100$ fs and $I_{\mathrm{pu}}=1.0,1.2, \ldots, 9.8,10 \times$ $10^{14} \mathrm{~W} / \mathrm{cm}^{2}$. The maps show results after bilinear bilinear interpolation.
} 
The final states are energy-dependent rather than intensity-dependent.

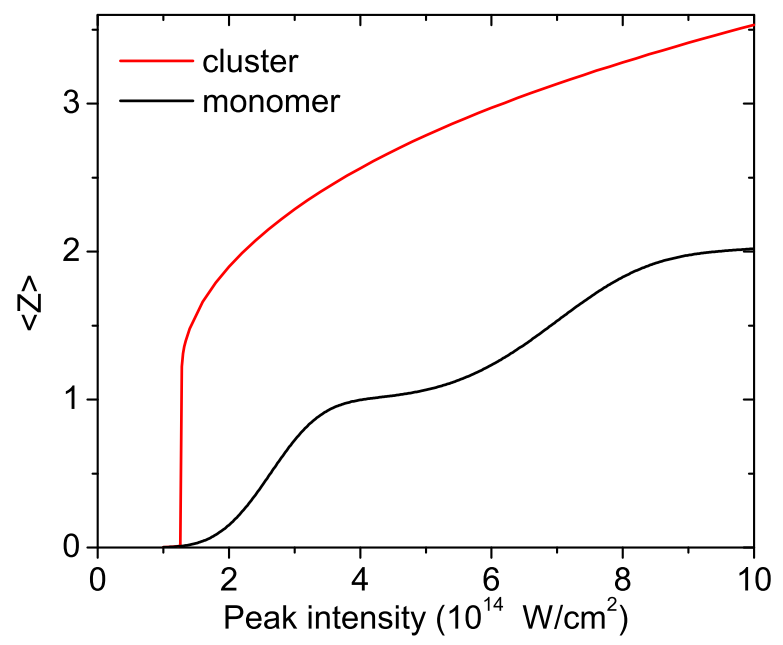

Figure 2.6: Charge state of Ar cluster (red) and monomer (black) at $t=50 \mathrm{fs}$ for an $800 \mathrm{~nm}, 40 \mathrm{fs}$ pump pulse vs. pump intensity. For $t \gtrsim 50 \mathrm{fs}$, the clustered plasma is in an equilibrium state in our model.

Fig. 2.6 shows $Z_{c}$ and $Z_{m}$ as a function of $I_{\mathrm{pu}}$ for a $40 \mathrm{fs}$ FWHM Gaussian pulse. A steep rise of $Z_{c}$ occurs at $I_{\mathrm{pu}} \sim 1.27 \times 10^{14} \mathrm{~W} / \mathrm{cm}^{2}$. At a first glance, one may relate this to ionization ignition (IONIG) model [68], which shows the onset and self-amplification of ionization process in clusters. In contrast, IONIG model treats small clusters (25-atom neon cluster), and the ignition starts when $\langle Z\rangle=1$. Therefore, the interpretation of IONIG model does not work in our situation. It turns out that the enhanced field inside the cluster is responsible for our result. When the intensity increases, $n_{e}$ rises as a result of the increased tunnel ionization rate. When $n_{e}$ sweeps upwards through $3 n_{\text {crit }}$, an enhanced field leads to an enhanced tunnel ionization rate 

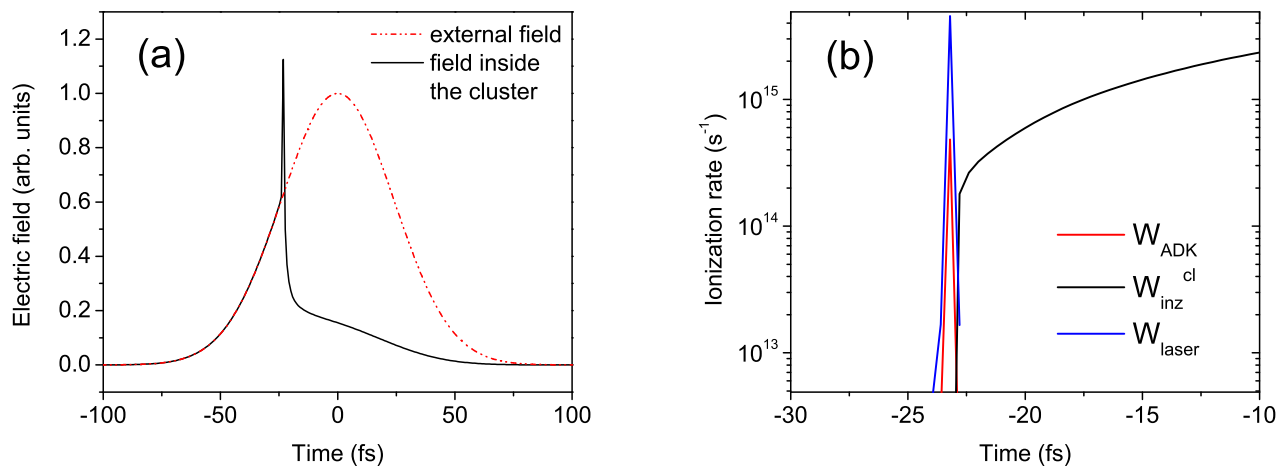

Figure 2.7: (a) Electric field inside the cluster (b) Rates of ADK ionization, collisional ionization, and laser-assisted ionization for $\mathrm{Ar} \rightarrow \mathrm{Ar}^{+}$during the short of period of field enhancement. $I_{\mathrm{pu}}=4.0 \times 10^{14} \mathrm{~W} / \mathrm{cm}^{2}, \lambda=800 \mathrm{~nm}, \tau=40 \mathrm{fs}$.

as well as an enhanced laser-assisted collisional ionization rate. An example of the enhanced field and ionization rates is shown in Fig. 2.7. At $t=-25 \mathrm{fs}$, the enhanced field raises the averaged quiver energy from $8 \mathrm{eV}$ to $31 \mathrm{eV}$, leaving most atoms ionized. As we'll show in Chapter 5 (p. 66), this behavior is not observed in experiments. The discrepancy may be caused by the assumption of a uniform top-hat radial density profile. A globally enhanced field disappears once a smooth boundary is used [47]. The field enhancement is then localized at a layer where $n_{e}=n_{\text {crit }}$. The contribution of this short time interval is insignificant for the final state when $I_{\mathrm{pu}}$ greatly exceeds the threshold intensity. For example, at $I_{\mathrm{pu}}=4 \times 10^{14} \mathrm{~W} / \mathrm{cm}^{2}$ and $40 \mathrm{fs}$, if we enforce an additional constraint of $E_{c} \leq E_{\text {ext }}$ to prevent field enhancement, the charge state changes from 2.56 to 2.58 , and the electron temperature remains the same.

The near field approximation requires cluster size $r \ll \lambda$. On the 
other hand, coulomb explosion (CE) may occur if $r$ is too small. The maximum radius $r_{\max }$ for which the optical field $E_{0}$ removes all ionized electrons from a cluster with uniform ion density $n_{i}$ can be shown [69] to be $r_{\max }=3 E_{0} / 4 \pi Z n_{i}|e|$. This corresponds to a radius of $0.14 \mathrm{~nm}$ for an argon cluster $\left(n_{i}=2.63 \times 10^{22} \mathrm{~cm}^{-3}\right)$, a typical intensity $10^{15} \mathrm{~W} / \mathrm{cm}^{2}$ and charge state $Z_{c}=4$. Since this size falls below the lattice constant of argon clusters $\left(a_{\mathrm{Ar}}=0.53 \mathrm{~nm}\right)$, we conclude that we are not in the $\mathrm{CE}$ regime.

Our model assumes that the electron population inside each cluster is a single-temperature Maxwellian. Any hot electrons that can be generated in the cluster are therefore neglected. Hot electrons may be generated by stochastic heating (SH) [69], which occurs if an electron accelerated in the laser field is temporarily shielded from the decelerating field when it enters the cluster. The electron may leave from the opposite side of the cluster in phase with the optical field, acquiring more kinetic energy. This process may happen multiple times as the electron moves back and forth through the cluster, causing an efficient heating. At our $I_{\mathrm{pu}}$, however, generation of hot electrons is unlikely. Even at relativistic intensities, it is often assumed that the relative fraction of hot electrons is below $10^{-4}[70,71]$. This condition guarantees that the hot electrons are unable to change $Z_{c}$ of all the ions significantly on a time scale of picosecond. The assumption of single-temperature Maxwellian distribution also relies on rapid electron-electron collisions, which equilibrate any deviation caused by heating and ionization. The collision is not rapid enough if $Z\left(v_{o s} / v_{e}\right)^{2} \gg 1[72]$, which may occur when $n_{e}$ sweeps through $3 n_{\text {crit }}$, result- 
ing in a super-Gaussian distribution. Nevertheless, this is a very brief time compared with the overall time of heating. After the resonance, the electrons quickly relax to a Maxwellian distribution.

The assumption of a uniform density profile keeps the problem from being two-dimensional. If the electric field is coupled with a non-uniform density profile, the field inside the cluster will have components in other directions [47], which may lead to anisotropy in the cluster dynamics.

Table 2.1 presents a comparison of $Z_{c}$ from a 3D microscopic particlein-cell (MPIC) code [73] to $Z_{c}$ calculated by our model for $800 \mathrm{~nm}, 100 \mathrm{fs}$ pulses. The difference between our result and that from MPIC simulation is $<$ $20 \%$. Considering the enormous difference in terms of computational time and resources, we therefore feel that our model is well suited to interpret various laser-cluster experiments.

Table 2.1: Comparison of $Z_{c}$ in Ref. [73] to $Z_{c}$ computed by our model for 100 fs pump pulses

\begin{tabular}{cccc}
\hline \hline Intensity $\left(\mathrm{W} / \mathrm{cm}^{2}\right)$ & $1 \times 10^{15}$ & $3 \times 10^{15}$ & $1 \times 10^{16}$ \\
\hline $\mathrm{Z}_{c}($ MPIC simulation) & 5.63 & 6.80 & 7.52 \\
$\mathrm{Z}_{c}$ (our model) & 4.71 & 6.41 & 8.20 \\
\hline \hline
\end{tabular}

To probe the charge state and electron temperature in an expanding cluster is challenging. In Ref. [74] the temperature for clusters of $r=5 \mathrm{~nm}$ irradiated by $1.3 \times 10^{15} \mathrm{~W} / \mathrm{cm}^{2}, 100 \mathrm{fs}, 800 \mathrm{~nm}$ pulses is found to be $130 \mathrm{eV}$ from time-of-flight measurements assuming a Maxwellian distribution. Our model gives a temperature of $61 \mathrm{eV}$. This is not bad if we consider that the emitted 
electrons are usually from the energetic part of the temperature distribution.

\subsection{D isothermal expansion model}

In our expansion model [50-52], cluster electrons are treated as isothermal due to their high heat conductivity. We assume different ion species have the same flow velocity $u(r, t)$. Without this assumption, a kinetic approach is in general required to describe plasma expansion together with recombination.

The flow velocity $u(r, t)$ is determined by the following equation:

$$
\frac{d u}{d t}=-\left(\sum_{Z=0}^{\infty} n_{i}(Z)\right)^{-1} \frac{T_{e}}{m_{i}} \frac{\partial n_{e}}{\partial r} .
$$

The continuity equation for ions with charge state $Z$ is given by

$$
\frac{\partial n_{i}}{\partial t}+\frac{1}{r^{2}} \frac{\partial}{\partial r}\left(u n_{i} r^{2}\right)=-R
$$

where

$R=\left[S(Z)+\alpha_{3}(Z) n_{e}\right] n_{e} n_{i}(Z)-n_{e}\left[S(Z-1) n_{i}(Z-1)+\alpha_{3}(Z+1) n_{e} n_{i}(Z+1)\right]$

The electron temperature equation is

$$
\begin{array}{r}
\frac{d}{d t}\left(\frac{3}{2} T_{e} \int n_{e} r^{2} d r+\int r^{2} d r\left[\frac{m_{i}}{2} u^{2} \sum_{Z=1}^{\infty} n_{i}(Z)\right]\right) \\
=\int r^{2} d r\left(\sum_{Z=0}^{\infty} \varepsilon_{i}(Z) \alpha_{3}(Z+1) n_{e}^{2} n_{i}(Z+1)-\varepsilon_{i}(Z) S(Z) n_{e} n_{i}(Z)\right) .
\end{array}
$$

Ions are driven by the ambipolar electric field generated by electron

pressure as described on the right-hand side in Eq. 2.18. Collisional energy 
relaxation between electrons and ions is negligible for the time we consider. The source term $R$ on the right-hand side of Eq. 2.19 describes the gain and loss of ion number due to three-body recombination and collisional ionization for each ion species. The right-hand side in Eq. 2.20 is the energy change due to recombination/ionization. The cluster is assumed to be spherically symmetric with the density of each ion species and electron temperature taken from our ionization/heating model. The equations were solved numerically using a Lagrangian solver with a staggered radical grid to obtain $n_{i}(Z), u, T_{e}$.

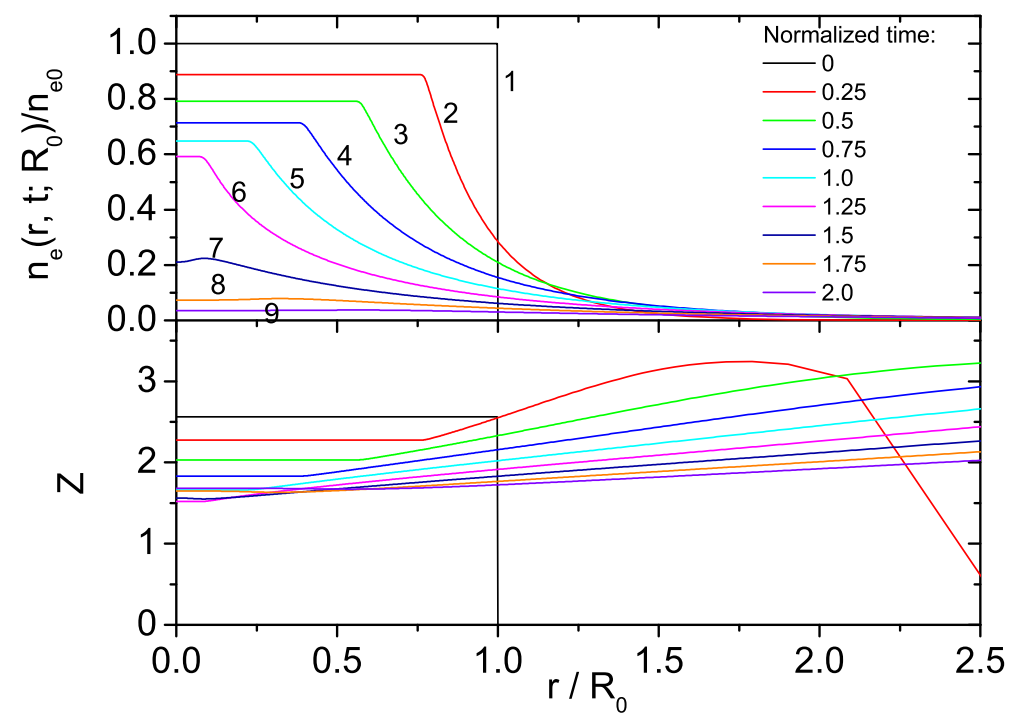

Figure 2.8: Snapshots of electron density profile and charge state profile during the expansion for a cluster of initial radius of $7 \mathrm{~nm}$. The initial ion population and electron temperature are calculated using model in previous section with laser parameters described in Fig. 2.3. The time for each curve is 1: $0 \mathrm{fs}, 2$ : $145 \mathrm{fs}, 3: 291 \mathrm{fs}, 4: 436 \mathrm{fs}, 5: 582 \mathrm{fs}, 6: 727 \mathrm{fs}, 7: 872 \mathrm{fs}, 8: 1018 \mathrm{fs}, 9: 1163 \mathrm{fs}$. 


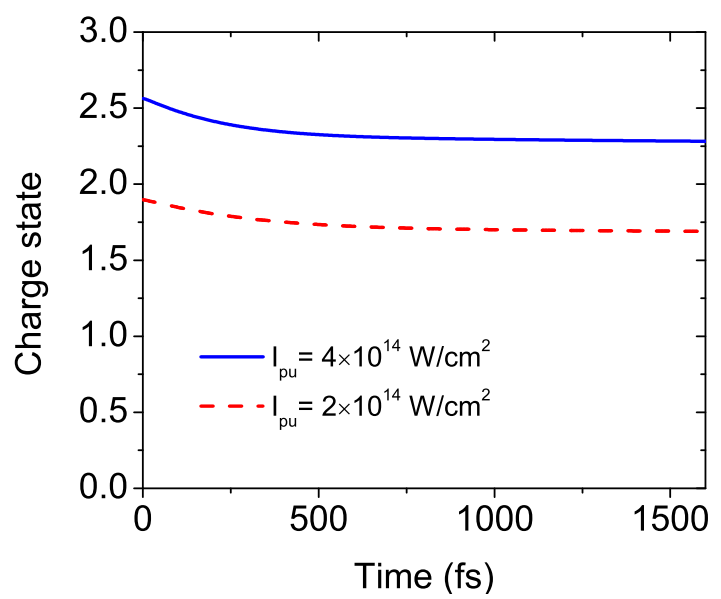

Figure 2.9: Evolution of $Z_{c}$ during the expansion for a cluster of $7 \mathrm{~nm}$ radius subject to a $40 \mathrm{fs}, 800 \mathrm{~nm}$ pulse with two different peak intensities.

Snapshots of a normalized electron density profile $n_{e} / n_{0}$ and charge state profile obtained by solving Eqs. 2.18-2.20 are shown in Fig. 2.8. The initial electron density is $n_{\mathrm{e} 0}=6.7 \times 10^{22} \mathrm{~cm}^{-3}$, which is $\sim 40$ times $n_{\text {crit }}=1.7 \times$ $10^{21} \mathrm{~cm}^{-3}(800 \mathrm{~nm})$. We obtained $Z_{c}=2.56$ and $T_{e}=23.4 \mathrm{eV}$ at $t=0$ from the ionization/heating model. The corresponding characteristic expansion time $\hat{t}=r / C s_{0}=582 \mathrm{fs}$, where $C s_{0}=\sqrt{Z_{0} T_{0} / m_{i}}$ with $m_{i}$ the ion mass. The expansion begins with a uniform density and charge state profile (see curves at $t=0)$. The snapshots for $t=145-727$ fs show the propagation of the inner front of the rarefaction towards the center of the cluster. Although the ion density at the center remains the same, the decrease of temperature breaks the balance, and recombination wins over collisional ionization, causing $Z_{c}$ to drop slowly. At other positions where $n_{i}$ drops as a result of expansion, the recombination cannot balance the collisional ionization, causing $Z_{c}$ to increase. 
Once the front reaches the center, $n_{e}$ drops rapidly, and $Z_{c}$ starts to rise. Despite the change of charge state profile, the overall average charge state changes slightly during the expansion, as shown in Fig. 2.9. This is also true for a weakly ionized cluster. The dotted line shows a calculation for a weakly ionized cluster with $Z_{c}=1.9, T_{0}=16.6 \mathrm{eV}$ prepared by $I_{\mathrm{pu}}=2.0 \times$ $10^{14} \mathrm{~W} / \mathrm{cm}^{2}$.
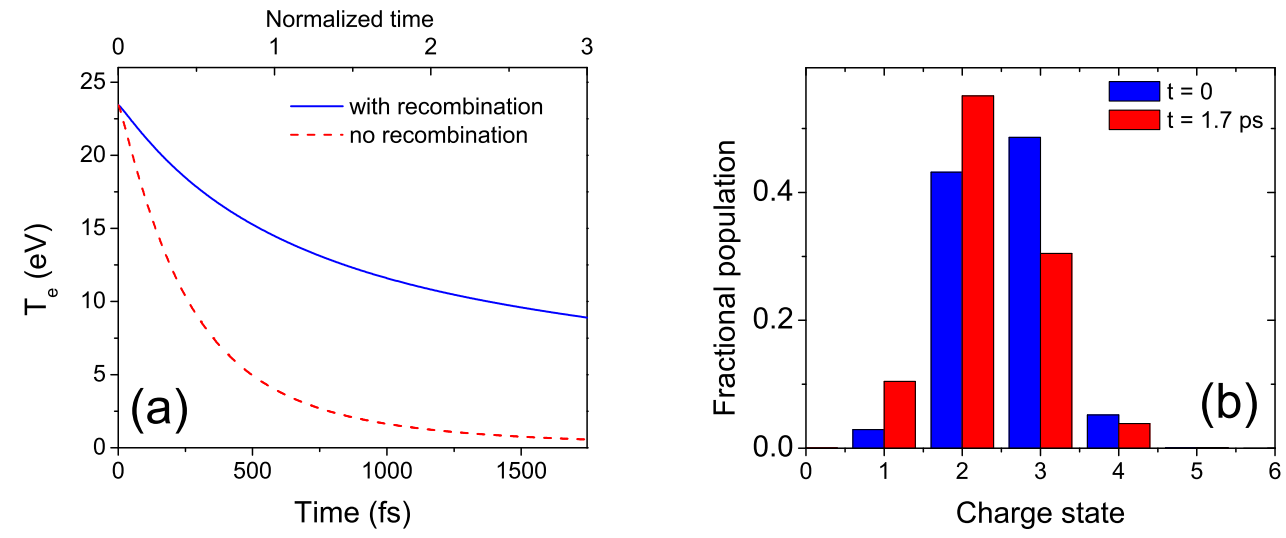

Figure 2.10: (a) Evolution of electron temperature during an isothermal expansion for a clustered plasma with initial radius of $7 \mathrm{~nm}$ with recombination/ionization (solid line) and without recombination/ionization (dashed line). (b) Charge state distribution at $t=0$ and $t=1.7$ ps.

The inclusion of recombination is essential for a realistic temperature evolution. As Fig. 2.10(a) shows, the temperature decays rapidly if recombination is not included. If that happens, the resulted high collision frequency will cause strong absorption, contradicting experimental observations. Fig. 2.10(b) shows the charge state distribution before the expansion and at $t=1.7$ ps. Most of the energy gain are from recombination with $\mathrm{Ar}^{3+}$. 


\subsection{Polarizability}

Once the electron density profile is known, we follow the procedure used in Refs. $[47,50]$ to find the cluster polarizability.

The electric near field equation is

$$
\nabla \cdot(\epsilon \nabla \varphi)=0
$$

where the local dielectric constant is $\epsilon_{c}=1-\omega_{p}^{2} /[\omega(\omega+i \nu)], \omega_{p}$ and $\nu$ are now radius-dependent. The electric field is $\mathbf{E}=-\nabla \varphi$. We seek a solution of the electric potential $\varphi(r, t)$ in the following form:

$$
\varphi(r, \theta)=-E_{0} f(r) \cos (\theta)
$$

Substituting back in Eq. 2.21, we get

$$
\frac{1}{r^{2}} \frac{d}{d r}\left(r^{2} \epsilon \frac{d f}{d r}\right)-\frac{2 \epsilon}{r^{2}} f=0
$$

This ODE is solved numerically. The polarizability of the cluster is related to $f\left(r, \tau ; R_{0}\right)$ by

$$
\alpha\left(\tau, R_{0}\right)=\frac{1}{3} \int_{0}^{\infty}(\epsilon-1)\left(\frac{\partial f}{\partial r}+2 \frac{f}{r}\right) r^{2} d r
$$

Fig. 2.11 shows the real and imaginary part of the polarizability of a cluster. The real part shows a positive contribution during initial $200 \mathrm{fs}$ and an asymptotic value after $\sim 1$ ps. 

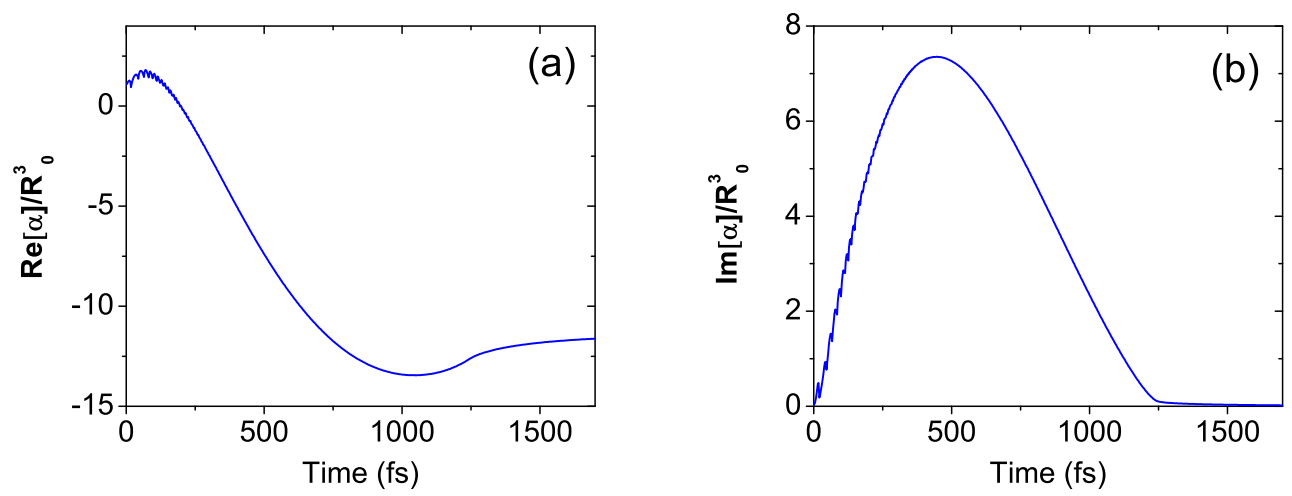

Figure 2.11: The real and imaginary part of the polarizability. The laser parameters and cluster parameters are the same as those in Fig. 2.8. The noise appears in the first 200 fs can be removed by reducing the step size in time in the expansion simulation. 


\section{Chapter 3}

\section{Properties of a cluster jet}

\subsection{Clusters in gas jets}

A gas jet produces atomic/molecular beams by allowing a high-pressure gas source with backing pressure $P_{0}$ to flow into a low-pressure ambient background with pressure $P_{b}$ through a small orifice. The flow may reach supersonic speed behind the throat if $P_{0} / P_{b}$ exceeds a critical value $G=((\gamma+1) / 2)^{\gamma /(\gamma-1)}$ $(<2.1$ for all gases), where $\gamma$ is the adiabatic index [75]. As the gas expands, the thermal energy is converted into kinetic energy of the flow. As a result, the gas becomes super-saturated; clusters bonded by Van-der-Waals force are formed.

Sonic and conical nozzles are commonly used in supersonic gas jets. Having no constraint on the flow behind the orifice, a sonic nozzle produces a beam with a half opening cone angle $\alpha_{0}=35.7^{\circ}$ for monoatomic gas [76]. Clusters generated under different conditions are often characterized by the Hagena parameter [77]. For sonic nozzles, it is given by

$$
\Gamma^{*}=k \frac{d^{0.85}}{T_{0}^{2.29}} P_{0},
$$

where $k$ is a gas species-dependent parameter ( $k=1650$ for argon gas), $d$ is the nozzle orifice diameter in $\mu \mathrm{m}, T_{0}$ is the temperature in Kelvin, and $P_{0}$ 
is the backing pressure in mbar. The lateral expansion in a conical nozzle is restricted by nozzle walls. The flow field (streamline) from a conical nozzle is similar to that from a sonic nozzle with a diameter $d_{\text {sonic }}=d \tan \left(\alpha_{0}\right) / \tan (\alpha)=$ $0.7 d / \tan \alpha$, where $\alpha$ is the expansion half angle. Thus the Hagena parameter for a conical nozzle ${ }^{1}$ is $[77]$

$$
\Gamma^{*}=k \frac{(0.74 d / \tan \alpha)^{0.85}}{T_{0}^{2.29}} P_{0} .
$$

Cluster size $\langle N\rangle$ is often estimated from $\Gamma^{*}$ by the scaling law derived from size measurements for $10^{3} \lesssim \Gamma^{*} \lesssim 10^{4} \quad[77]$ :

$$
\langle N\rangle=33\left(\frac{\Gamma^{*}}{1000}\right)^{2.35} .
$$

For $10^{4}<\Gamma^{*}<10^{6}$, another scaling law is suggested [80]:

$$
\langle N\rangle=100\left(\frac{\Gamma^{*}}{1000}\right)^{1.8} .
$$

Continuous jets were often used in early days. Pulsed jets gained popularity since they reduce gas consumption and do not require vacuum pumps of high capacity. In our experiments, clusters were generated from a series-9 or series-99 pulsed solenoid valve driven by an Iota One pulse valve driver (Parker Hannifin, General Valve division). Fig. 3.1 shows an image of the gas jet and the schematic of the series-9 valve. The valve is sealed by an O-ring (series-9) or a metal gasket (series-99). When the valve is in operation, the

\footnotetext{
${ }^{1}$ Despite the wide use of $\Gamma^{*}$, the formula is sometimes used incorrectly. e.g. the coefficient 0.74 is missing [78]; $\alpha$ is mistakenly stated as the opening angle of the cone [79].
} 

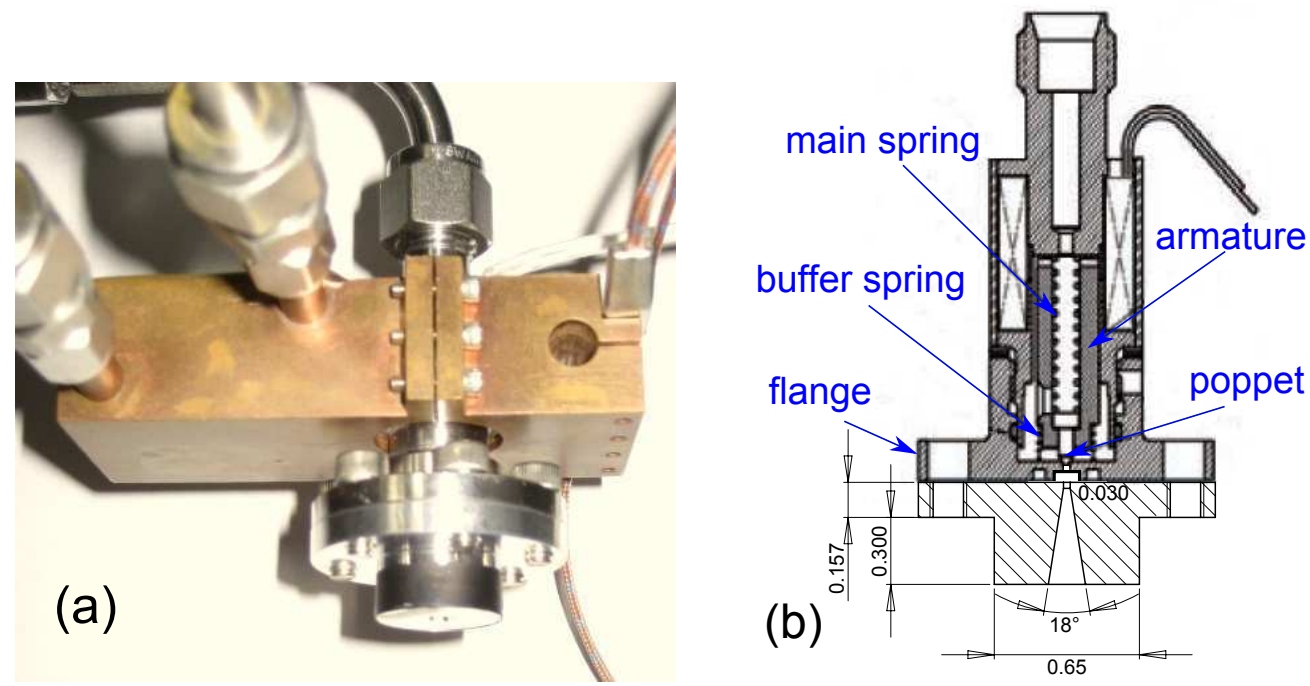

Figure 3.1: (a) Image of the gas jet. (b) Schematic of the solenoid valve and the home-made nozzle. All units are inches.

magnetic field produced by a high voltage $(\sim 300 \mathrm{~V})$ draws the armature into the solenoid, thus opening the valve. After $250 \mu \mathrm{s}$, the voltage is reduced to $28 \mathrm{~V}$ to hold the armature and to prevent from heating up of the coil. A main spring pushes a Kel-F poppet and seals the orifice when the voltage is off. A buffer spring sitting between the armature and the orifice helps to minimize possible damage caused by the collision between the poppet and the orifice. The performance of the valve depends critically on the distance between the upper wall and lower seat. The adjustment is achieved by tightening or loosening the flange from the valve body (series-9), or by placing a gasket of proper thickness (series-99). The flange from General Valve is of cylindrical aperture with a diameter of $0.31^{\prime \prime}(790 \mu \mathrm{m})$. A conical nozzle is preferred for efficient generation of clusters. Two configurations - replacing the flange with 
a homemade conical nozzle and attaching a conical nozzle to the flange - were tested. The latter configuration was adopted because the Rayleigh scatter indicated the latter had a more uniform profile than the former especially at high pressure. To study the temperature influence on the cluster formation, a series-99 valve is closely surrounded by a copper block as shown in Fig. 3.1(a). A zig-zag pattern was cut inside the block to maximize the interior surface. Nitrogen gas cooled by liquid nitrogen flows into the copper block and cools the copper block, which serves as a temperature reservoir for the valve body. A type- $\mathrm{T}$ thermocouple attached to the copper block measures the reservoir

temperature. Attempts were made to cool the copper block by flowing liquid nitrogen. However, the small inner diameter of tubing $\left(1 / 16^{\prime \prime}\right)$ prevented the liquid nitrogen from flowing under gravity.

\subsection{Atomic density measurement}

Gas density can be calculated from the pressure, inferred from the fluorescence [81], or measured by transverse interferometry. The density is related to the pressure at room temperature by

$$
N_{\text {tot }}\left[\mathrm{cm}^{-3}\right]=3.3 \times 10^{16} P[\text { torr }] .
$$

A Michelson interferometer was used to measure the gas density, as shown in Fig. 3.2. A 50/50 beamsplitter divided the beam into reference and probe, which were combined after reflection from mirror M1 and M2, respectively. The beam profile near the gas jet was captured by a triggered CCD camera 


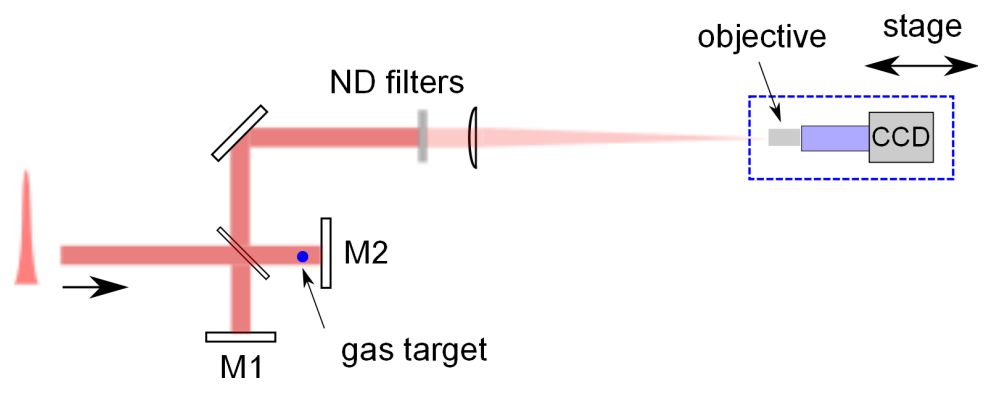

Figure 3.2: Schematic setup for gas atomic density measurement

after a singlet lens and a microscopic objective. The object plane was found by translating the CCD until a sharp edge of the gas jet was observed. Mirror M1 was slightly tilted to produce fringes of proper density. Fig. 3.3 shows the interferogram and phase shifts extracted using a Fourier transform method [82] for the gas jet at $t=0.8 \mathrm{~ms}$ with an opening time of $0.8 \mathrm{~ms}$.
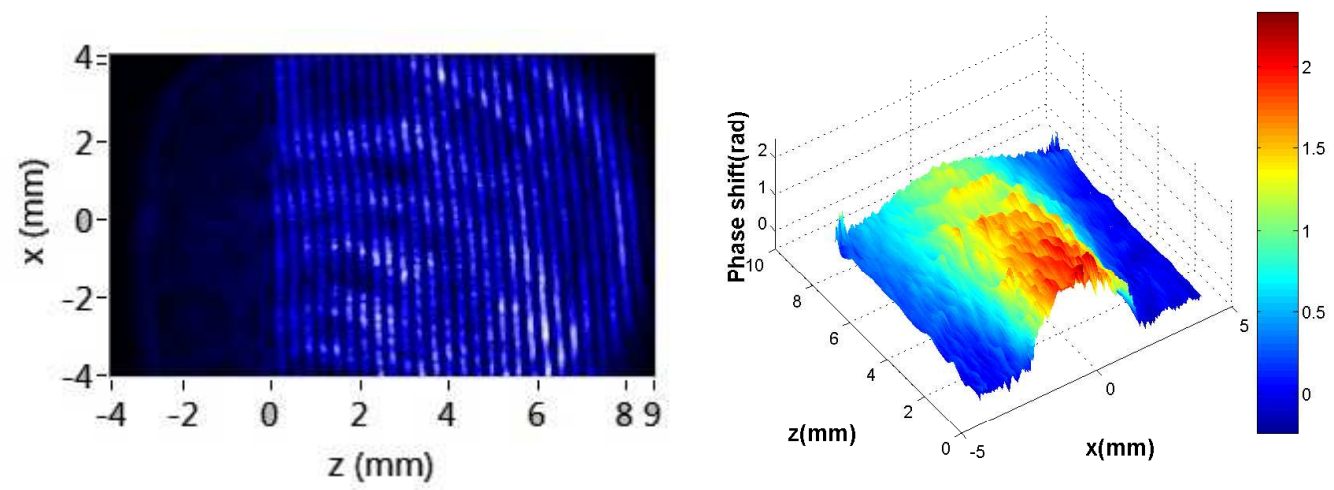

Figure 3.3: (Left) Sample interferogram at 500 psi, $0.8 \mathrm{~ms}$ after the valve opens. $z=0$ is the edge of the gas jet. (Right) Extracted phase shift $2 \Delta \phi(x, z)$.

The pulse passed the target twice with a time difference $\sim 200 \mathrm{ps}$, for which we can safely assume the gas density is frozen. We divided the 
extracted phase shift by two to find the single-pass phase shift $\Delta \phi$. If we neglect the small difference of the polarizability of an argon atom in clusters and a free argon atom, the change of refractive index due to the gas jet is $\Delta n_{\text {jet }}=2 \pi N_{\text {tot }} \alpha$, where $\alpha$ is the polarizability of a free atom. For an object of cylindrical symmetry, the phase shift $\Delta \phi(x)$ is

$$
\Delta \phi(x)=\frac{2 \pi}{\lambda} \int \Delta n_{\text {jet }} d y=\frac{(2 \pi)^{2} \alpha}{\lambda} \cdot 2 \int_{x}^{R} N_{\text {tot }}(r) \frac{r}{\sqrt{r^{2}-x^{2}}} d r .
$$

$N_{\text {tot }}(r)$ is found using Abel inversion,

$$
\frac{(2 \pi)^{2} \alpha}{\lambda} N_{\mathrm{tot}}(r)=-\frac{1}{\pi} \int_{r}^{R} \frac{d \Delta \phi(x)}{d x} \frac{1}{\sqrt{x^{2}-r^{2}}} d x .
$$

We follow an inversion procedure based on fast Fourier transforms (FFT) [83]. $\Delta \phi(x)$ is written in cosine expansion,

$$
\Delta \phi(x)=a_{0}+\sum_{k=0}^{k_{\max }} a_{k} \cos \frac{k \pi x}{R} .
$$

The density may be written as

$$
N_{\text {tot }}(r)=\frac{\lambda}{8 \pi \alpha R} \sum_{k=0}^{k_{\max }} k a_{k} g_{k}\left(\frac{r}{R}\right),
$$

where

$$
g_{k}(\rho)=\frac{2}{\pi} \int_{0}^{\left(1-\rho^{2}\right)^{1 / 2}} \frac{\sin \left(k \pi \sqrt{t^{2}+\rho^{2}}\right)}{\sqrt{t^{2}+\rho^{2}}} d t .
$$

A lineout of $\Delta \phi(x)$ at $1 \mathrm{~mm}$ below the gas jet and its super-Gaussian fit are shown in Fig. 3.4. Analytically, $k_{\max }$ should be infinity. A small $k_{\max }$ is usually sufficient for the desired accuracy. We choose two different $k_{\max }$ to invert the raw and fitted data. For $k_{\max }=25, N_{\text {tot }}$ inverted from the raw data 

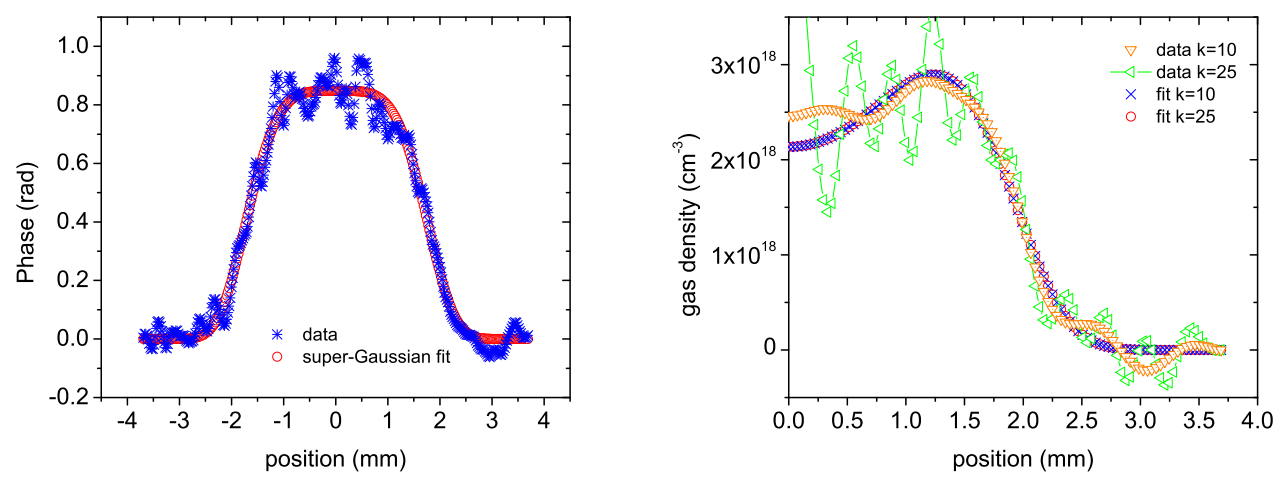

Figure 3.4: (Left) Single-pass phase shift $\Delta \phi(x)$ at $z=1 \mathrm{~mm}$. (Right) Gas density obtained after FFT-based Abel inversion for noisy data and fitted curve with two different $k_{\max }$.

has strong oscillations at the center. For $k_{\max }=10$, the result is similar to that from the fitted data. Thus this method can filter the data and complete Abel inversion together. For the fitted data, $k_{\max }=10$ and 25 yield almost the same curve.

The time variation of the line phase shifts through the center of the gas jet for a pulsed gas jet is presented in Fig. 3.5. The phase shift grows superlinearly initially, fluctuates slightly for a duration of $\sim 1 \mathrm{~ms}$, and then reaches a constant value. The time when the superlinear growth stops depends on the backing pressure. Similar features were reported [84], where the fluctuation after superlinear growth was attributed to the bouncing of the armature after it reaches the upper wall of the value. The minimum time for a sudden open jet to establish a steady state may be calculated using the model in Ref. [85]. 


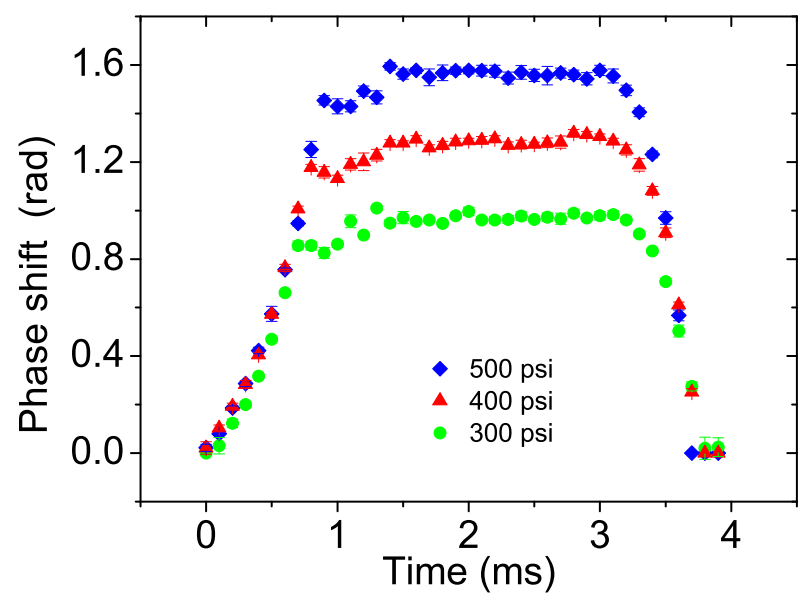

Figure 3.5: Sub-ms time-dependent phase shift of a neutral gas jet

\subsection{Rayleigh scattering measurement}

The Rayleigh scattering differential cross section for $S$-polarized light is $[56]$

$$
\frac{d \sigma}{d \Omega}=\frac{k^{4} r_{c}^{6}}{2}\left|\frac{\varepsilon_{c}-1}{\varepsilon+2}\right|^{2},
$$

where $r_{c}$ is the cluster size, $\varepsilon_{c}$ is the dielectric constant of a cluster, and $k$ is the wave number of an incident laser. In contrast, $d \sigma / d \Omega=0$ for $P$-polarized light at $90^{\circ}$. Rayleigh scatter is often used to monitor the onset of cluster formation. In experiments, a biconvex lens imaged Rayleigh scattering profile at $90^{\circ}$ of the incident $400 \mathrm{~nm}, S$-polarized pulses to a CCD. Fig. 3.6 shows the Rayleigh scatter vs. time $t$ after the valve opens. The error bar indicates the standard deviation of 30 shots, which is partly because the incident pulse has an energy fluctuation $\sim 10 \%$. The scattered signal rises rapidly between 


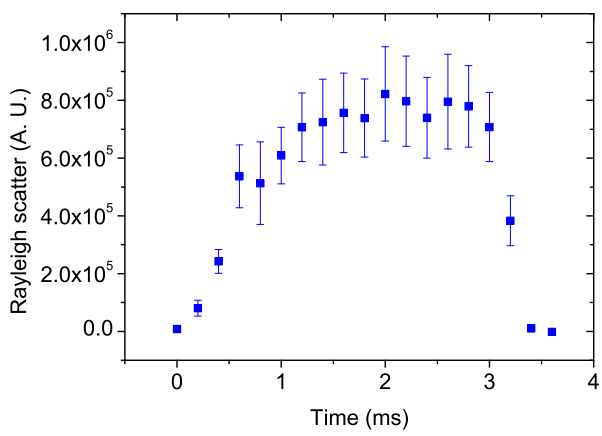

Figure 3.6: Rayleigh scatter vs. time after the valve opens

0-0.8 ms, increases slightly, and remains high for the rest of the time until the valve closes.

The dependence of Rayleigh scatter on the pressure $P$ is often used to verify the scaling law. The signal scales as $P^{\beta}$, where $\beta=3.35$ if Eq. 3.3 applies [86]. Two assumptions are implied in such an argument ${ }^{2}$ : (1) the cluster fraction $f_{c}$ is constant; (2) the total atomic density $N_{\text {tot }} \propto P$. We'll show the influence of pressure on $f_{c}$ in the next chapter. The latter is usually valid at the center of the jet when the valve is operated at typical conditions and in the steady state. As shown in Fig. 3.7, different $\beta$ can be found before the gas jet reaches the steady state.

In an attempt to observe Rayleigh scatter from expanding clusters with $800 \mathrm{~nm}, P$-polarized pump pulses and $400 \mathrm{~nm}, S$-polarized probe pulses, we noticed that the Rayleigh scatter may be buried by the fluorescent emission.

\footnotetext{
${ }^{2}$ With these assumptions, Rayleigh scatter signal $\propto N_{c} N^{2} \propto N_{\text {tot }} N \propto N_{\text {tot }} \Gamma^{2.35} \propto P^{3.35}$.
} 


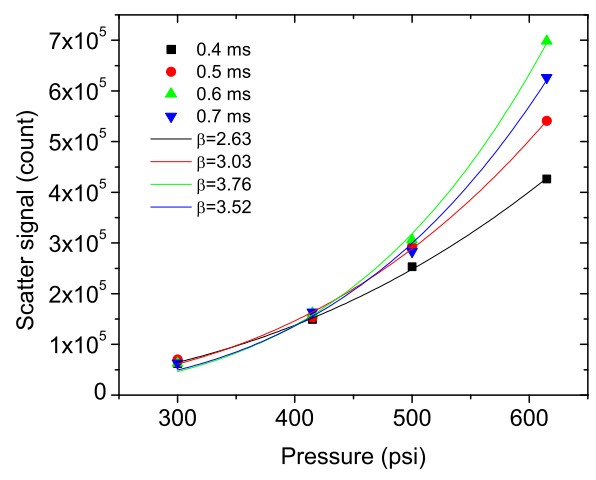

Figure 3.7: Rayleigh scatter vs. backing pressure
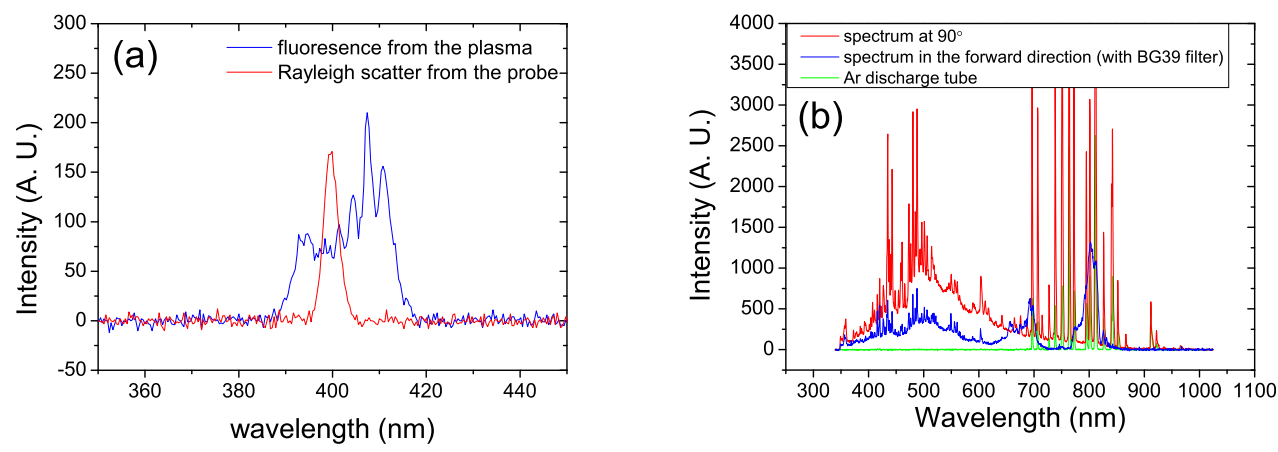

Figure 3.8: (a) Fluorescence and Rayleigh scatter. (b) Fluorescent spectra from laser-produced plasma.

The spectra of Rayleigh scatter and fluorescent emission after a $400 \mathrm{~nm}$ bandpass filter are shown in Fig. 3.8(a). The latter is the continuum emission of dense plasmas, which is observed both in $90^{\circ}$ and forward direction, as shown in Fig 3.8(b). Second harmonic of the pump pulse in the gas jet may be generated by four-wave mixing of the static field from charge separation and the pump field [87]. In our case, we did not observe second harmonic. 


\section{Chapter 4}

\section{Measurement of cluster mass fraction}

\subsection{Introduction}

Not all atoms condense into clusters in a cluster jet. The degree of condensation can be described by the cluster mass fraction $f_{c} \equiv \frac{N N_{c}}{N_{m}+N N_{c}}$, where $N_{m}$ and $N_{c}$ denotes the number density of monomers and clusters, respectively, $N$ is the number of atoms in the cluster. Using the size distribution $g(N)$, it may be defined as

$$
f_{c} \equiv \frac{\int_{N>N_{\min }} N g(N) d N}{\int N g(N) d N} .
$$

The term cluster may be restricted to $N \geq N_{\min }$, where $N_{\min }$ depends on the method used, if smaller particles show the same properties as monomers. Cluster mass fraction is also called cluster mole fraction, clustering fraction, clustering ratio, condensed fraction, or condensation degree. It has proven to be an elusive quantity to measure, calculate or estimate accurately for pulsed supersonic jets typical of intense laser experiments $\left(r_{c} \gtrsim 5 \mathrm{~nm}, N_{\text {tot }} \gtrsim 10^{18} \mathrm{~cm}^{-3}\right)$, although various techniques that are restricted to much lower $r_{c}$ and/or $N_{\text {tot }}$ - electron impact ionization/photoionization mass spectroscopy [88], fluorescence excitation spectroscopy [89], cluster-helium beam scattering [79], electron diffraction [90], surface scattering [91] — or to molecular gas jets - 
Table 4.1: Cluster mass fraction measured by various methods. For reference, $N=1.1 \times 10^{5}$ for an argon cluster of $10 \mathrm{~nm}$ radius.

\begin{tabular}{lllll}
\hline \hline Ref. & Quantities & Gas species & $f_{c}$ & $\langle N\rangle$ \\
\hline$[88]$ & mass spectra & $\mathrm{CO}_{2}$ & 0.9 & $\sim 500$ \\
{$[89]$} & fluorescence excitation spectra & $\mathrm{Xe}$ & $\sim 0.75$ & $\sim 600$ \\
{$[79]$} & He atom scattering & $\mathrm{Ar}$ & 0.11 & 27 \\
{$[90]$} & electron diffraction & $\mathrm{Ar}$ & 0.8 & $\sim 750$ \\
{$[91]$} & surface scattering & $\mathrm{Ar}$ & 1.0 & $\sim 4000$ \\
{$[92]$} & Raman spectra & $\mathrm{CO}_{2}$ & 0.13 & $\sim 1000$ \\
\hline \hline
\end{tabular}

Raman scattering [92] — have been used to determine $f_{c}$ in tenuous or molecular clustered gases. Table 4.1 presents some results from these measurements. Most of these techniques require the beam to be extracted into a separate high vacuum chamber where measurements take place, thus forbidding an in situ characterization. It is sometimes assumed [93] without clear justification that all atoms in a jet are clustered (i.e. $f_{c}=1.0$ ). On the other hand, theoretical models of cluster nucleation using liquid drop nucleation rate theory [94] or the Smoluchowski coagulation equation [95] show wide variations of $f_{c}$ in gas jets, typically $f_{c}<0.5$ in room temperature jets. Moreover, most results are measured at the center of the gas jet. The interaction region in many intense laser-cluster experiments is placed near the edge of a gas jet, where $f_{c}$ may vary widely, to avoid severe refraction and absorption $[29,48] . f_{c}$ also depends on time $t$ after opening the valve, temperature $T_{0}$, pressure $P_{0}$ and nozzle geometry, although no empirical formulas exist for estimating these dependencies. Direct knowledge of $f_{c}$ is critical not only to the understand- 
ing of nucleation and condensation, but also to accurate interpretation of a wide variety of laser-cluster experiments including X-ray emission [96], phasematching harmonic generation [29] and accelerating ions [8] where $f_{c}$ has not been verified in an independent experiment. In addition, in situ measurement of $r_{c}$ using Rayleigh scatter $[80,86]$ relies indirectly on knowledge of $f_{c}$, since the Rayleigh scatter signal is proportional to $f_{c} r_{c}^{6}$. Direct measurement of $r_{c}$ is needed in many situations where the scaling law using Hagena's parameter is unreliable, including: (i) jets that have not yet reached steady state [85]; (ii) jets with ambiguous nozzle geometric parameters - e.g. a poppet displacement comparable or less than the orifice diameter $d$ may result in a reduced effective throat diameter [97]; or (iii) jets with atypical nozzle shapes that do not match the gas flow [98]; or atypical expansion angle or pressure [99]. These situations illustrate the wide need for an in situ experimental determination of $f_{c}$.

\subsection{Method: fs-time-resolved refractive index}

Here we determine $f_{c}(\boldsymbol{r}, t)$ at position $\boldsymbol{r}$ within, and time $t$ after firing, a cluster jet by measurement of the jet refractive index $n_{\text {jet }}(\boldsymbol{r}, t ; \tau)[50,100]$. Here $\tau$ denotes time delay after a pump pulse ionizes both monomers and clusters. Ionization of monomers (and possibly very small clusters ${ }^{1}$ ) causes $n_{\text {jet }}$ to drop immediately during the pump by an amount proportional to $N_{m}$,

\footnotetext{
${ }^{1}$ The size distribution obeys exponential decay for small clusters and log-normal distribution for large clusters $(\langle N\rangle \gtrsim 500)$. Clusters of small sizes are absent if $\langle N\rangle$ is large [88].
} 
before large ionized clusters contribute significantly to $n_{\text {jet }}$. The latter begin to contribute after several hundred fs, when their internal electron density drops to the critical density $n_{\text {crit }}=m_{e} \omega_{\mathrm{pr}}^{2} / 4 \pi e^{2}$ of the probe pulse (frequency $\omega_{\mathrm{pr}}$ ) as a result of hydrodynamic expansion. The details of this evolution, however, are not relevant here. The present method relies only on the asymptotic value of $n_{\text {jet }}$, reached after clusters expand into uniform monomer plasma, causing a second delayed drop in $n_{\text {jet }}$ that is proportional to $N N_{c}$. Thus $n_{\text {jet }}$ evolves in two steps corresponding to monomer and cluster contributions, allowing recovery of $f_{c}$. Since $\tau$ is measured in fs and $t$ in ms, the jet may be considered frozen in $t$ throughout the index measurement.

The probe phase shift is related to refractive index by

$$
\Delta \phi_{\mathrm{pr}}=\frac{2 \pi}{\lambda} \int\left[n_{\mathrm{jet}}(z)-n_{\mathrm{gas}}(z)\right] d z
$$

where $z$ denotes distance from the gas jet entrance, $n_{\text {gas }}(z)=1+2 \pi N_{\text {tot }}(z) \alpha$ is the jet's refractive index (including both monomers and clusters) before ionization, and $\alpha$ is the atomic polarizability of a free $\mathrm{Ar}$ atom, which we assume to be the same for an atom in a cluster ( $\alpha$ in solid Ar is only $6 \%$ smaller than for free atoms [101]). The monomer plasma contribution to $n_{\text {jet }}(\mathrm{z})$ is

$$
n_{m}(z)=1-\frac{1}{2} \frac{Z_{m}\left(1-f_{c}(z)\right) N_{\mathrm{tot}}(z)}{n_{\mathrm{crit}}}
$$

where $Z_{m}$ is the charge state of the monomers, and we have assumed a tenuous plasma $\left(N_{\text {tot }} \ll n_{\text {crit }}\right)$. The asymptotic cluster contribution to $n_{\text {jet }}(z)$ is an additional term $-Z_{c} f_{c}(z) N_{\text {tot }}(z) / 2 n_{\text {crit }}$, where $Z_{c}$ is the charge state of the ionized 
clusters at $\tau>1$ ps. Here, for simplicity, we assume $f_{c}(z)$ is constant along the short interaction length. With this assumption, the monomer-induced phase shift is

$$
\Delta \phi_{m}=-\frac{\pi}{\lambda}\left[\frac{Z_{m}\left(1-f_{c}\right)}{n_{\text {crit }}}+4 \pi \alpha\right] \int N_{\text {tot }}(z) d z
$$

for $Z_{m} \geq 1$. The asymptotic cluster contribution is

$$
\Delta \phi_{c}=-\frac{\pi}{\lambda} \frac{Z_{c} f_{c}}{n_{\text {crit }}} \int N_{\text {tot }}(z) d z
$$

The cluster fraction $f_{c}$ is related to the observables $\Delta \phi_{m}, \Delta \phi_{c}$ by

$$
f_{c}=\frac{\left(Z_{m}+4 \pi \alpha n_{\text {crit }}\right) \Delta \phi_{c}}{Z_{c} \Delta \phi_{m}+Z_{m} \Delta \phi_{c}}
$$

This result is independent of the integrated density profile, which therefore need not be characterized. In this sense, the method is self-referencing.

\subsection{Experiment: frequency domain interferometry}

We performed a measurement of 2-step index evolution using multishot frequency-domain interferometry (FDI), in which $n_{\text {jet }}(\tau)$ for each delay $\tau$ is measured on a separate shot [50]. In this experiment, clusters were formed by condensation in a room temperature pulsed supersonic argon jet inside a vacuum chamber. A series-9 pulsed solenoid valve from Parker Hannifin, backed by 600 or 800 psi pressure, opened for $1.5 \mathrm{~ms}$ to admit gas into a conical nozzle with orifice diameter $d=750 \mu \mathrm{m}$ and half expansion angle $11^{\circ}$. Fig. 4.1 shows schematics of the FDI experiment. $800 \mathrm{~nm}, 100$ fs pulses from a $10 \mathrm{~Hz}$ Ti:sapphire laser system were split into two beams. Pump pulses (400 


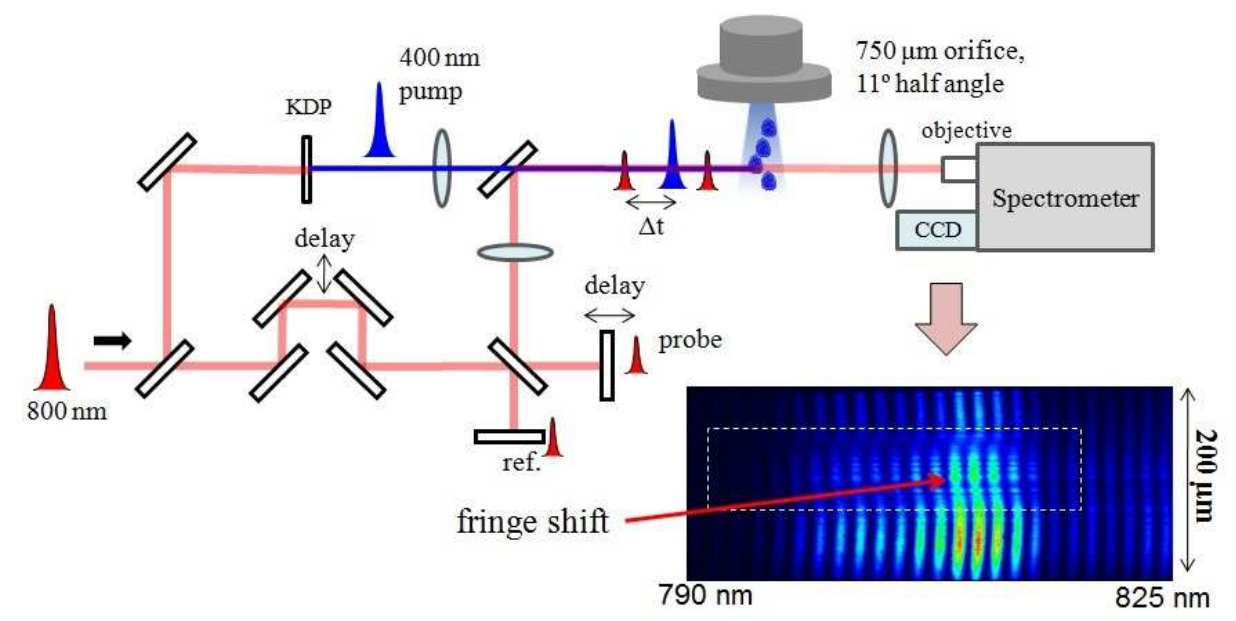

Figure 4.1: Schematic set-up for fs-time-resolved measurement of $\mathrm{n}_{\text {jet }}(\tau)$.

$\mathrm{nm}, 100 \mathrm{fs}$ ) were generated by frequency doubling one beam in a $1 \mathrm{~mm}$ thick, type-I phase-matched KDP crystal. The pump pulses, focused with a singlet lens, had a beam diameter $\left(1 / \mathrm{e}^{2}\right.$ in intensity) of $40 \mu \mathrm{m}$. The focused pump intensity was $4.4 \times 10^{15} \mathrm{~W} / \mathrm{cm}^{2}$. An $800 \mathrm{~nm}$ pulse was split by a Michelson interferometer into a co-propagating probe pulse and a reference pulse with a fixed temporal separation of $1.6 \mathrm{ps}$. The probe pulse was focused by a singlet lens to the pumped region of the jet and its diameter was approximately $150 \mu \mathrm{m}$. The probe and reference pulses were imaged from the exit of the jet onto the entrance slit of the spectrometer by a focusing lens and a microscopic objective. The two pulses interfered in the spectral domain after dispersion on the grating. A CCD camera recorded the interferogram. The probe pulse was modulated by the plasma-cluster medium and gained additional phase. The phase shift with respect to the reference beam at the center of the pump 
region for each delay is extracted from the interferogram by a Fourier analysis program. A sample interferogram is shown in Fig. 4.1. In addition to the expected phase shifts, the fringes show a curvature outside the pumped region. Similar curvatures observed in later experiments could be eliminated by optimizing the spatial overlap of the probe and reference.

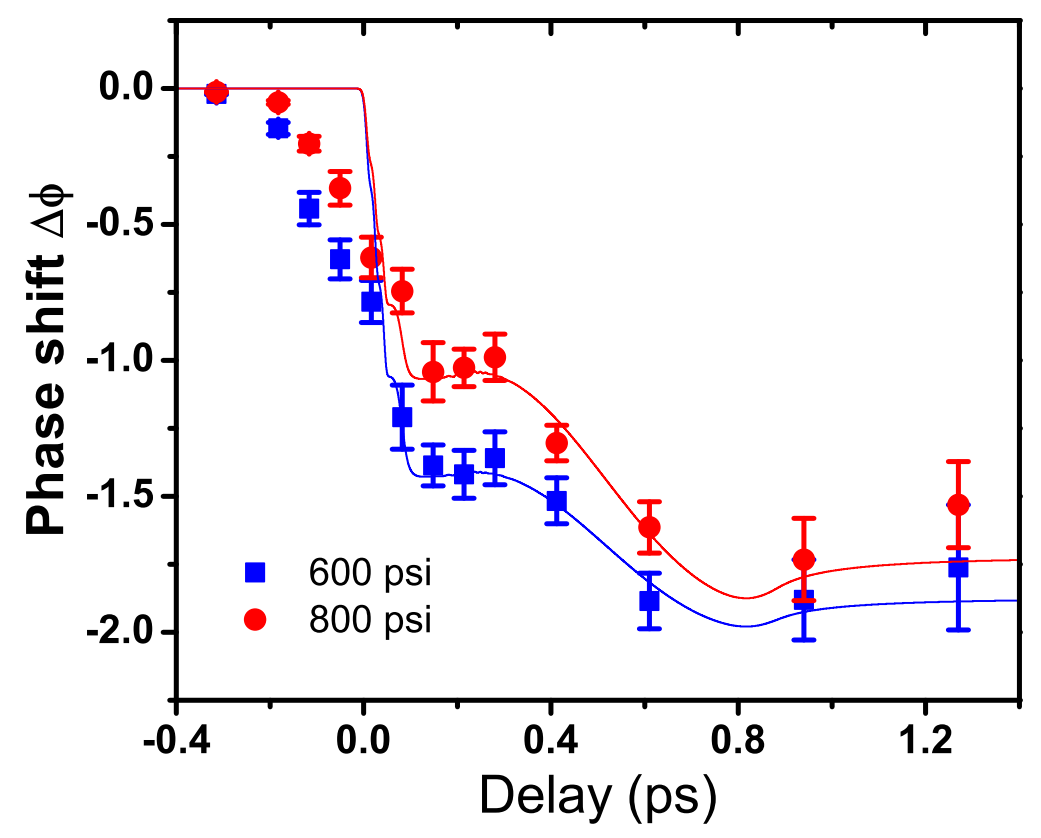

Figure 4.2: Fs-time-resolved phase shifts measured by FDI. Each data point is 20 shots average. The error bar is the standard deviation. The lines are refractive index $n_{\text {jet }}$ using size $r=20 \mathrm{~nm}$ and $f_{c}=0.15$ and 0.2 , respectively.

Fig. 4.2 shows $\Delta \phi(\tau)$ at two different backing pressures. An initial drop and a delayed slow drop are observed. The solid lines are calculated time-dependent refractive index for a cluster of $20 \mathrm{~nm}$ radius. The phase 
shifts have a larger delayed drop at 800 psi than at 600 psi, indicating a higher $f_{c}$. Because the measurements were taken at the edge of the gas jet, where $N_{\text {tot }} \propto P$ does not hold, the asymptotic phase shift at 800 psi is smaller than that at 600 psi.

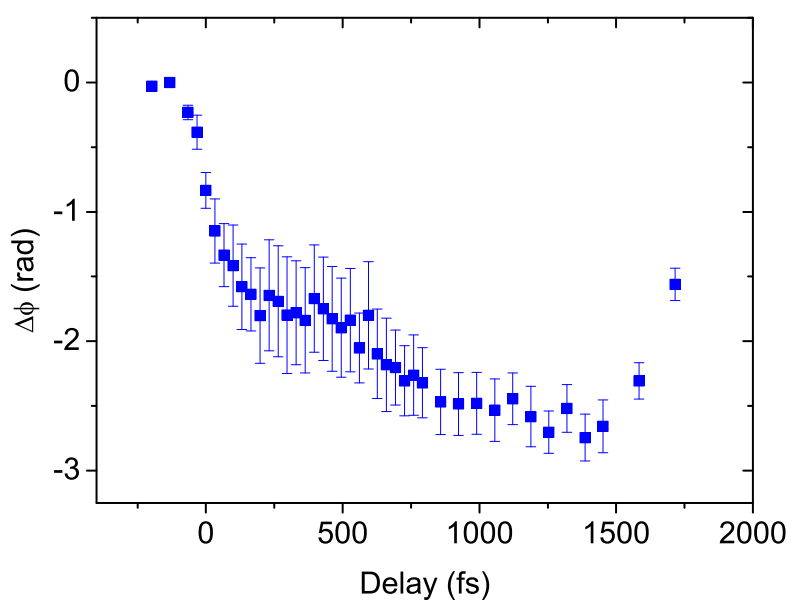

Figure 4.3: Multi-shot averaged time-resolved phase shifts measured by FDI.

After a major renovation of the laboratory, a similar setup was built with a new laser system. Fig. 4.3 shows the measurement with a 40 fs, $I_{\mathrm{pu}} \sim$ $3 \times 10^{14} \mathrm{~W} / \mathrm{cm}^{2}$ pulse and 350 psi backing pressure. The setup was identical to that in Fig. 4.4, except for the absence of SF10 glass behind the Michelson interferometer. "Two steps" are observed again. The separation between reference and probe is $\sim 1.6 \mathrm{ps}$. The reference pulse starts to pick up phase shift from the plasma after $t=1.6 \mathrm{ps}$, causing the rise of the phase shift in Fig. 4.3. The large error bar (standard deviation of 50 shots) is possibly caused by the energy fluctuation. The laser was operated at a reduced output 


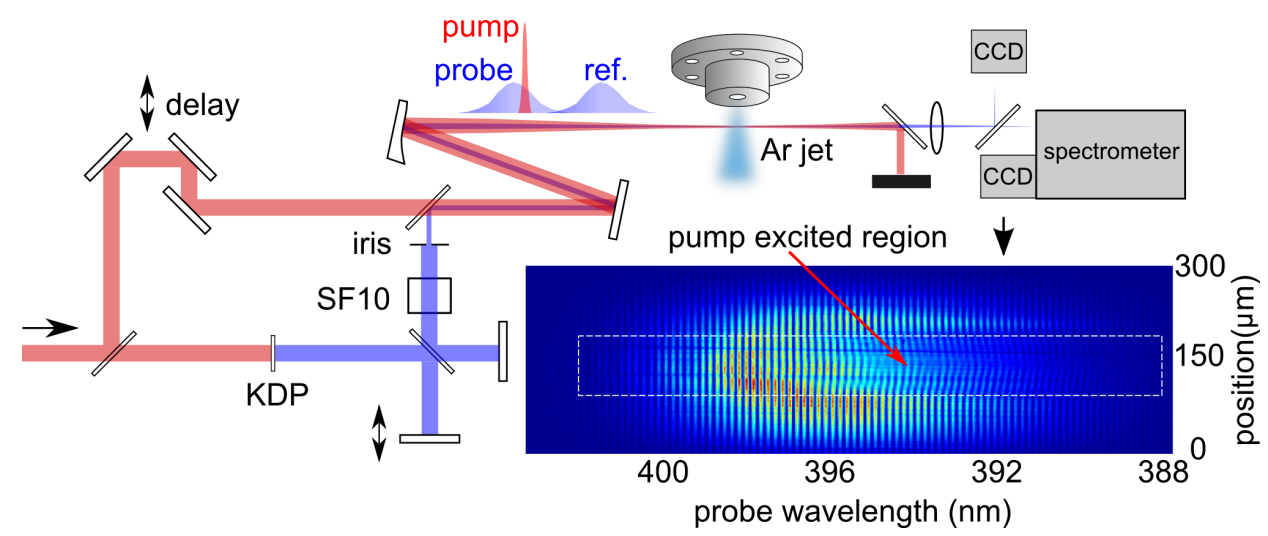

Figure 4.4: Schematic set-up for single-shot, fs-time-resolved measurement of cluster gas jet refractive index $n_{\text {jet }}(\tau)$. Frequency-domain hologram (lower right) records pump-induced phase alterations imprinted on probe.

power, which caused an energy fluctuation of 9\% (standard deviation of 200 shots). Our $I_{\mathrm{pu}}$ falls between the ionization threshold and intensity needed for $Z_{m}=1$. Thus $Z_{m}$ is sensitive to the shot-to-shot fluctuation of the laser intensity. In addition, this measurement is time consuming. To overcome these problems, we switched to a single-shot frequency domain holography (FDH) measurement.

\subsection{Experiment: frequency domain holography}

\subsubsection{Experimental setup and alignment procedure}

Here, by measuring $n_{\text {jet }}(\tau)$ in a single shot, sensitivity to shot-to-shot fluctuations was eliminated. Moreover, the greatly increased measurement speed enables convenient scans of the dependence of $f_{c}$ on jet parameters $(e . g$. $t$, backing pressure, temperature). In our experiments, a solenoid-controlled 
valve (General Valve Series 9, opening time $\sim 1 \mathrm{~ms}$, backing pressure 100-700 psi at RT) controlled a supersonic argon jet emerging from a conical nozzle (750 $\mu \mathrm{m}$ orifice, $9^{\circ}$ half angle, $\left.\Gamma^{*} \approx 1.3 \times 10^{5}\right)$ into a $10^{-3}$ Torr vacuum. The valve driver was synchronized with the laser system; the repetition rate was reduced to $0.25 \mathrm{~Hz}$ to maintain a high vacuum. At this background pressure, no phase shift was observed from ionized residual atoms. Fig. 4.4 shows the FDH schematic. A $10 \mathrm{~Hz}$ train of $800 \mathrm{~nm}, 40$ fs Ti:S laser pulses with $10^{6}: 1$ peakto-background ratio at 1 ps from the peak (higher at longer times) was split into pump and probe beams. Probe pulses were frequency doubled in a 500 $\mu \mathrm{m}$ KDP crystal, split by a Michelson interferometer into probe and reference pulses separated by $3 \mathrm{ps}$, each chirped to $\sim 2$ ps duration in $2 \mathrm{~cm}$ SF10 glass, and irised down to ensure a large focused spot with uniform wavefront. These $400 \mathrm{~nm}$ pulses recombined with the $800 \mathrm{~nm}$ pump at a dichroic beam splitter, with reference pulse leading and probe spanning delays $-0.75<\tau<$ 1.3 ps. An off-axis parabola focused the 3 co-propagating pulses $\left(f^{\#}=60\right.$ for pump) through an off-center chord of the gas jet about $1 \mathrm{~mm}$ from the nozzle where the interaction length was $\sim 2 \mathrm{~mm}$ (compared to Rayleigh length $z_{R}=10 \mathrm{~mm}$ ), and probe absorption not too severe. Reference and probe pulses were frequency filtered and relay-imaged from the gas jet exit to the entrance slit of an imaging spectrometer, with the pumped region (monitored by a CCD camera) centered on the slit. They interfered at the spectrometer's detection plane, where a second CCD camera recorded each frequency-domain hologram (see example in Fig. 4.4). A Fourier transform procedure [82, 102] 
then reconstructed the pump-induced temporal phase shift $\Delta \phi_{\operatorname{pr}}(\tau)$ of the probe along the pump propagation axis.

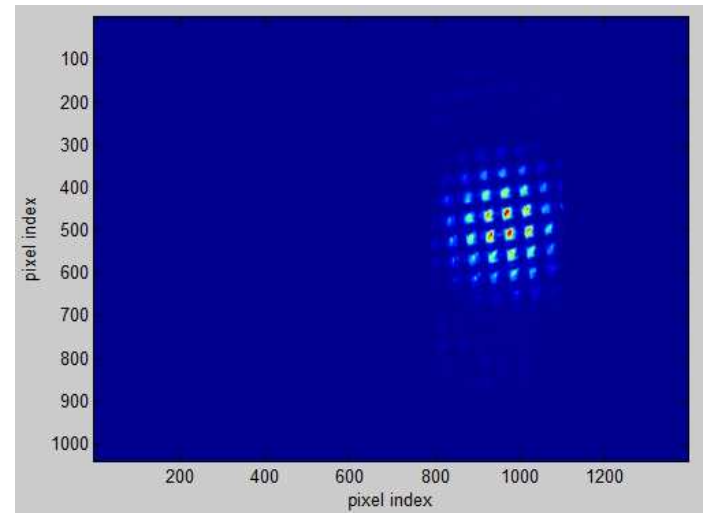

Figure 4.5: Image of the mesh (0.0011" wire diameter, 325 lines per inch) used to determine the object plane and to calibrate the magnification.

The detection system included a Jobin Yvon HR460 spectrometer (CzernyTurner configuration with negligible astigmatism) and a Basler Scout-f CCD (scA1400-17fm/fc, 12 bit of ADC bit depth, $6.45 \times 6.45 \mu \mathrm{m}^{2}$ pixel size). A helium discharge tube illuminated the entrance slit of the spectrometer with a slit width of $\sim 10 \mu \mathrm{m}$. We adjusted the position of CCD until the spectral lines gave the narrowest peaks on the CCD, indicating that the CCD was on the focal plane of the spectrometer. From the known spectral lines around 400 nm, the wavelength on each pixel was calibrated. A slit width of $\sim 30 \mu \mathrm{m}$ was used in experiments to admit more light without loosing fringe contrast. A reference target (wire mesh) was attached to the gas jet $\sim 2 \mathrm{~mm}$ behind the axis of the gas jet, $\sim 5 \mathrm{~mm}$ away from the axis transversely. To check the object plane, we translated the gas jet transversely until the laser illuminated 
the mesh. The spectrometer was set at 0 nm (i.e. the zero-order), permitting a 1:1 image of the entrance slit. The lens position was adjusted until the best image was found, as shown in Fig. 4.5. This guarantees the desired object plane and allows us to calculate the magnification of the imaging system.

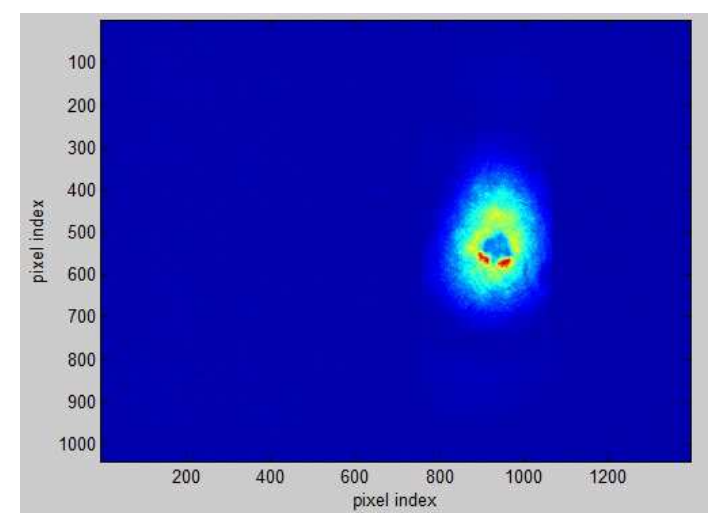

Figure 4.6: Image when the probe is behind the pump pulse.

For pump-probe experiments, an accurate temporal overlap of two pulses is the foundation for any further delay adjustment. Synchronization using electronic devices is typically limited to approximately one nanosecond ${ }^{2}$, which corresponds to $30 \mathrm{~cm}$ in the optical path length. A coarse temporal overlap was achieved by measuring and eliminating the difference of optical path lengths of the two pulses. Difference within a couple of centimeters or several millimeters would usually be achieved. A precise overlap was achieved by observing the phenomenon occurring when two pulses were temporal overlapped. If the two pulses are collinear and of the same wavelength, such as the

\footnotetext{
${ }^{2}$ For example, the rise time of a photodiode is $\sim$ ns; the bandwidth of an oscilloscope is $\sim \mathrm{GHz}$
} 
case of our probe and reference, spatial interference fringes are created only when two pulses are at least partially overlapped in time. For pulses of $<100$ fs, this corresponds to $\sim 20 \mu \mathrm{m}$ in the adjustment of the delay line. Thus a small travel step of the stage is required to catch the overlap. An effective method is to track the separation of two pulses from the spectral interference using a fiber spectrometer. The fringes can be observed if the separation of pulses is within several picoseconds. After the alignment of temporal overlap, one may adjust the delay line to achieve a proper fringe density in the hologram captured by the CCD. For two-color pump and probe, we utilized the pump-induced features imprinted in the probe pulse such as ionizationinduced refraction or blueshift of the probe spectrum. A spatial overlap of the focal spots of the two pulses was needed. Using the coarse temporal overlap, we found a delay when the probe was behind the ionizing pump pulse. As long as the delay is within the decay time of the plasma, which exceeds nanoseconds, the defocusing effect shows up in the probe beam when pump and probe are spatially overlapped. The defocusing effect can be viewed from the far-field image of the probe profile on a screen. Unstable features similar to burning fire caused by the refraction of the plasma can be seen. With the help of an imaging system, the pump-imprinted region was easily identified in a large focal spot created by an apertured probe pulse, allowing us to adjust this region to the center of the probe, as shown in Fig. 4.6. After that, an accurate temporal overlap was achieved by changing the delay line until the defocusing disappeared. 


\subsubsection{Phase reconstruction}

The spectral amplitudes $E_{\mathrm{pr}}(\omega)$ and phase shifts $\Delta \phi(\omega)$ were obtained from the hologram by a Fourier transform procedure [82]. An example is shown in Fig. 4.8. A linear mapping between $\omega$ and $t$ is only practical if the variation is slow. For a better temporal resolution, the temporal phase shifts were reconstructed from spectral phases, spectral amplitudes for both the probe and reference [102], which is given by

$$
\Delta \Phi(t)=\operatorname{Im}\left[\ln \frac{\int E_{\mathrm{pr}}(\omega) e^{i \phi_{r}(\omega)+i \Delta \phi(\omega)} e^{-i \omega t} d \omega}{\int E_{\mathrm{ref}}(\omega) e^{i \phi_{r}(\omega)} e^{-i \omega t} d \omega}\right] .
$$

In a discrete domain, the integral is found using FFT. We have

$$
\Delta \Phi\left(t_{n}\right)=\operatorname{Im}\left\{\ln \frac{\mathrm{FFT}\left[E_{\mathrm{pr}}(\omega) e^{i \phi_{r}(\omega)+i \Delta \phi(\omega)}\right]}{\mathrm{FFT}\left[E_{\mathrm{ref}}(\omega) e^{i \phi_{r}(\omega)}\right]}\right\},
$$

where the sampling time interval $\Delta t=2 \pi / N \Delta \omega$. One may get a smaller time interval by zero padding. However, the additional points do not provide additional information because zero padding in the frequency domain corresponds to interpolation in the time domain.

The spectral phase of the reference $\phi_{r}(\omega)$ is related to the group delay dispersion $\beta_{2}$ by $\phi_{r}=\beta_{2}\left(\omega-\omega_{0}\right)^{2}$. To determine $\beta_{2}$, we varied the delay $\tau$ of the pump pulse with respect to the probe pulse. The frequency $\omega$ where $\Delta \phi(\omega)$ started to drop was selected at each $\tau$. A plot of $\omega$ vs. $\tau$ is shown in Fig. 4.7. Since $\omega(t)=\omega_{0}+b t$, the slope of the linear fit gives the linear chirp rate $b=6.21 \pm 0.05 \mathrm{fs}^{-2}$. The linear chirp coefficient $a=1 / b$ is related to the group delay dispersion by $a=2 \beta_{2}\left[1+\beta_{2}^{-2}(\Delta \omega)^{-4}\right]$. Therefore, we determined $\beta_{2}=8.05 \times 10^{-27} \mathrm{~s}^{2}$. 


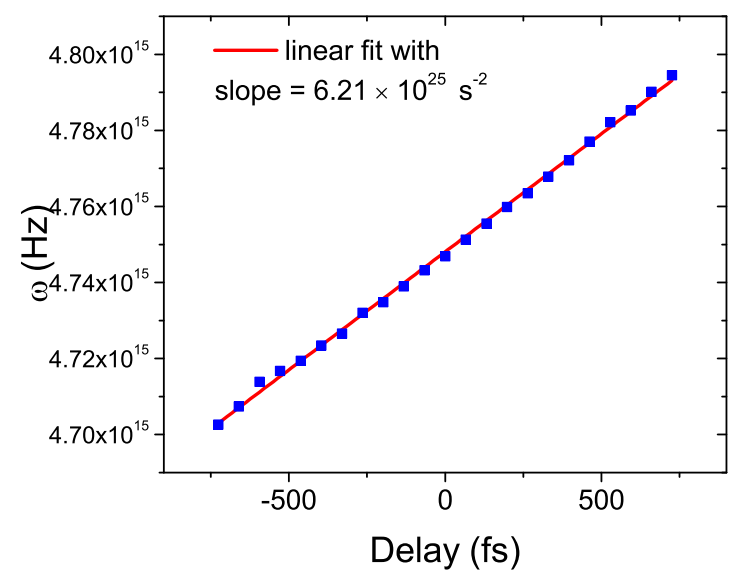

Figure 4.7: Calibration of the chirp parameter.
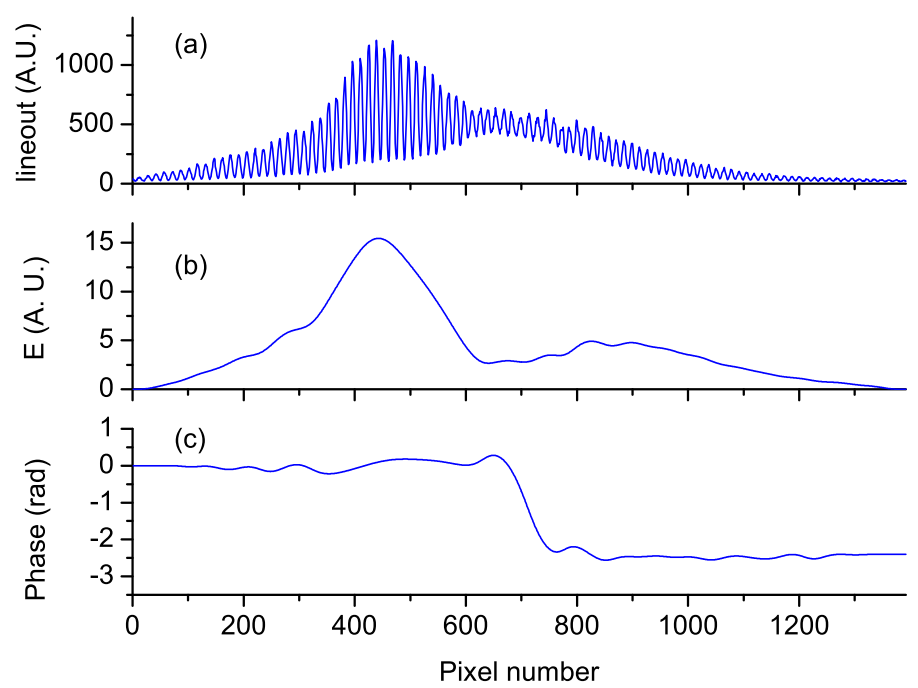

Figure 4.8: Spectral amplitude and phase extracted using Fourier transform method. 


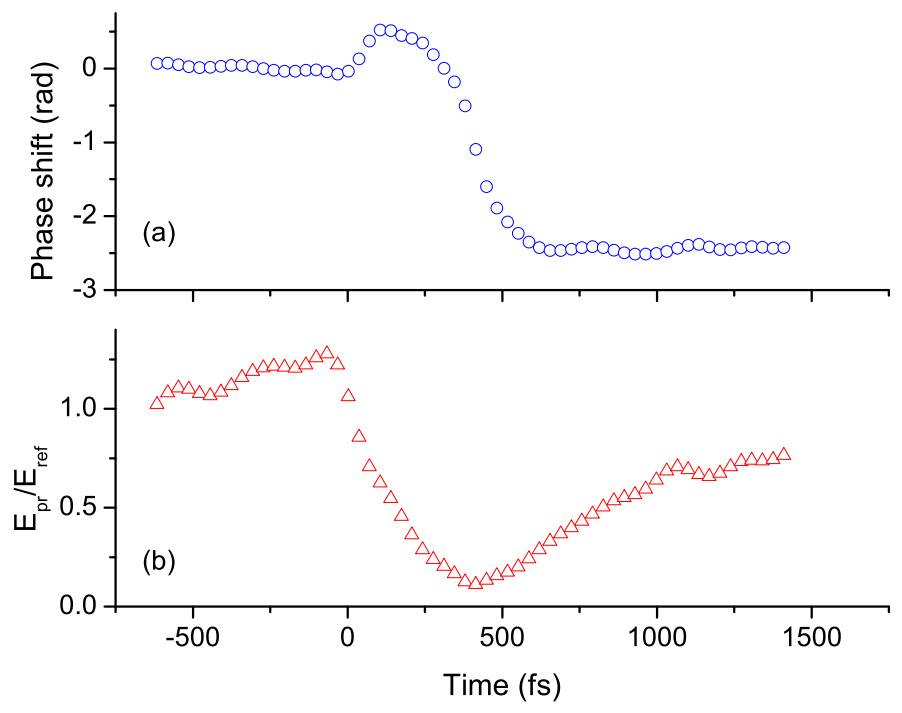

Figure 4.9: Reconstructed temporal phase shifts and $E_{\mathrm{pr}} / E_{\mathrm{ref}}$.

Fig. 4.9 shows the reconstructed real phase shift and $E_{\mathrm{pr}} / E_{\mathrm{ref}}$ for $I_{\mathrm{pu}} \sim$ $1 \times 10^{14} \mathrm{~W} / \mathrm{cm}^{2}$ through the center of the gas jet. In Fig. 4.8, $\Delta \phi(\omega)$ drops $\sim 2 \mathrm{rad}$ as a result of ionization. In contrast, $\Delta \phi(t)$ in Fig. 4.9 rises initially, drops slowly after several hundred of femtoseconds, and reaches an asymptotic value of $\sim 2$ rad eventually. Because the pump intensity is below the ionization threshold of argon monomer, the initial drop from monomer plasmas is absent. The flat phase shifts after $700 \mathrm{fs}$ indicate that recombination is negligible. The smooth rise from atomic dispersion to plasma dispersion suggests that all the clusters expand hydrodynamically and negligible electrons escape the cluster during the ionization. Otherwise, an immediate drop will be present. The transient of $E_{\mathrm{pr}} / E_{\mathrm{ref}}$ is caused by absorption of the probe by the expanding 


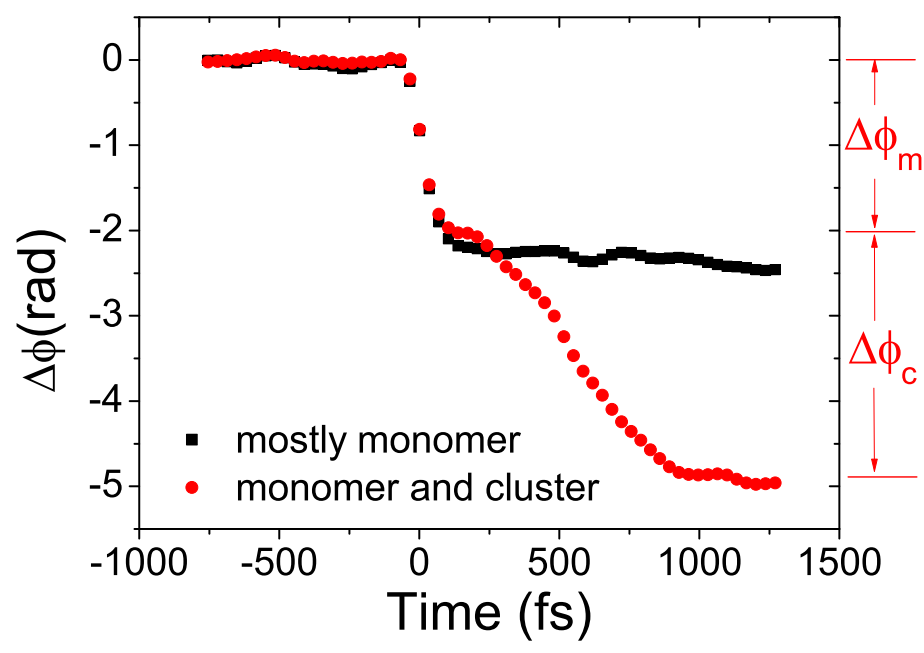

Figure 4.10: Two fs-time-resolved probe phase shifts $\Delta \phi_{\mathrm{pr}}(\tau)$, each measured in a single shot, at time $t=0.3 \mathrm{~ms}$ (black) or $0.8 \mathrm{~ms}$ (red) after opening of gas jet valve.

clusters. The error of $E_{\mathrm{pr}} / E_{\mathrm{ref}}$ is large for $t<-300 \mathrm{fs}$ or $t>1 \mathrm{ps}$. This is because at the leading or trailing edge, the signal-to-noise ratio is large. In addition, $E_{\text {ref }}$ and $E_{\text {pr }}$ were captured from different shots. That explains why apparently $E_{\mathrm{pr}} / E_{\mathrm{ref}}>1$ before ionization starts.

\subsubsection{Example of $f_{c}$ evaluation}

Fig. 4.10 shows fs-time-resolved phase shifts at two different time $t$ after the valve opens. The black curve shows $\Delta \phi_{\mathrm{pr}}(\tau)$ from pump-probing the jet at $t=0.3 \mathrm{~ms}$, before large clusters formed, in part because total atomic density was still low. $\Delta \phi_{\mathrm{pr}}(\tau)$ drops immediately by $\sim 2 \mathrm{rad}$ as monomers (and possibly very small clusters) ionize, then remains nearly constant. The red curve shows $\Delta \phi_{\mathrm{pr}}(\tau)$ from pump-probing the jet at $t=0.8 \mathrm{~ms}$, after clusters 
had formed. Two components can be identified. The fast component, which coincidentally has the same $\Delta \phi_{\mathrm{pr}}(\tau)$ as the black curve, is caused by ionization of monomers. The slow component comes from large expanding clusters, and reaches a steady state at $\tau \sim 1$ ps as clusters approach a uniform underdense plasma.

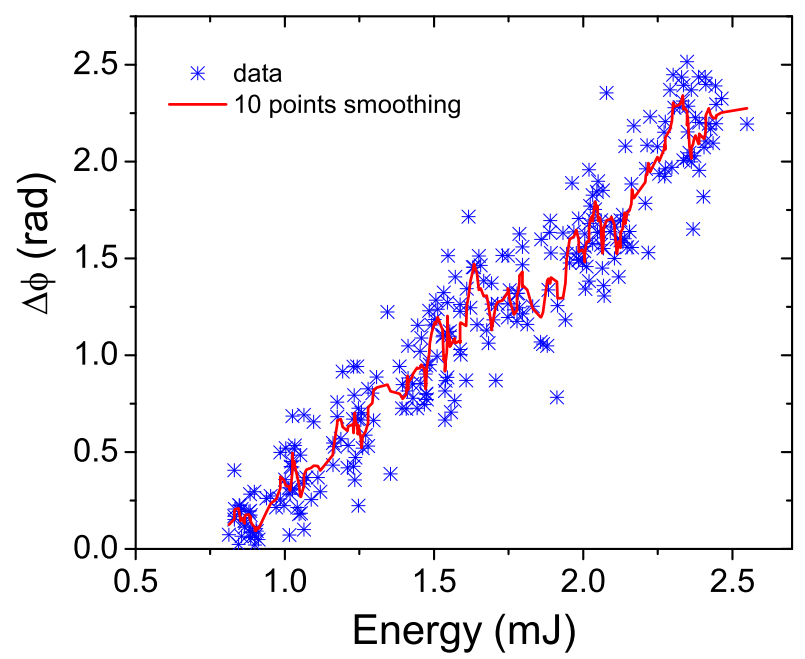

Figure 4.11: Phase shift vs. energy delivered to the gas jet.

We calculated $Z_{c}$ and $Z_{m}$, which are required in the evaluation $f_{c}$ with Eq. 4.6, using the model described in Chapter 2. For simplicity, we neglected the change of charge state during cluster expansion, which is $<10 \%$ according to our expansion model. We assumed the ensemble of clusters yield the same charge state after laser irradiation, or the ionization/heating is sizeindependent. This is a good approximation for nanometer-sized clusters [103]. The intensity was determined in-situ by varying pump energy and observing 


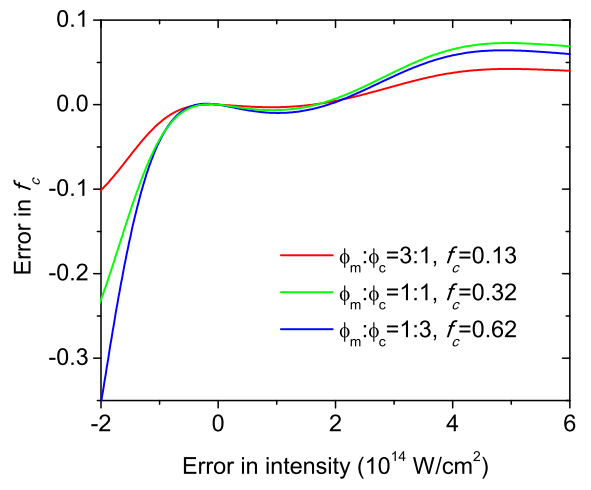

Figure 4.12: Error of $f_{c}$ caused by error in $I_{\mathrm{pu}}$

the appearance of monomer ionization. Fig. 4.11 shows the phase shifts from single-shot measurements vs. the laser energy. The red curve is a 10-point Savitzky-Golay smoothing curve. The energy was tuned by a $\lambda / 2$ plate and a thin film polarizer. Additional energy spread was caused by the fluctuation of laser energy. The gas jet was operated at similar conditions as when the black curve in Fig. 4.10 was measured. Therefore, cluster contribution was almost absent. A photodiode recorded the amount of energy leaking through a dielectric mirror. The photodiode was calibrated using an energy meter. The energy of $E=0.8 \mathrm{~mJ}$ at which phase shift appears corresponds to the threshold of $I_{\mathrm{pu}}=1.9 \times 10^{14} \mathrm{~W} / \mathrm{cm}^{2}$. A plateau is observed at approximately twice of that energy, consistent with predication of ADK model. By choosing $I_{\mathrm{pu}}=4 \times 10^{14} \mathrm{~W} / \mathrm{cm}^{2}$ in the $Z_{m}=1$ plateau between the 1 st and 2 nd ionization thresholds, we minimize sensitivity of the result to small uncertainty in $I_{\mathrm{pu}}$. At this intensity, we calculate $Z_{m}=1, Z_{c}=2.6$. With $\alpha=1.6982 \times 10^{-24}$ $\mathrm{cm}^{3}$ and $n_{\text {crit }}=6.875 \times 10^{21} \mathrm{~cm}^{-3}$, we find $f_{c}=0.42$ for the red curve in 
Fig 4.10. The sensitivity of the result to the error of $I_{\mathrm{pu}}$ is shown in Fig. 4.12. $f_{c}$ for three $\Delta \phi_{m} / \Delta \phi_{c}$ are given for a correct $I_{\mathrm{pu}}=4 \times 10^{14} \mathrm{~W} / \mathrm{cm}^{2}$. An underestimate of the intensity leads to an underestimate of $f_{c}$.

\subsubsection{Parameter Scans}

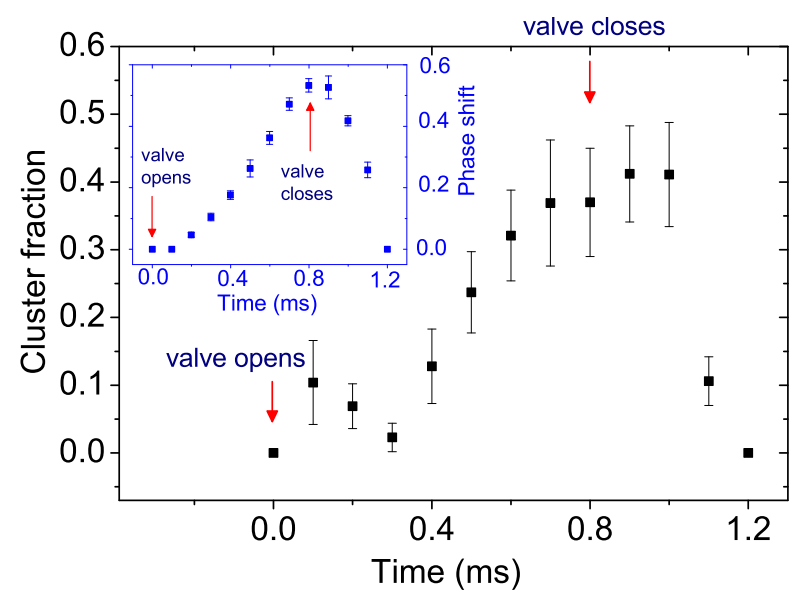

Figure 4.13: Cluster fraction $f_{c}$ vs. time $t$ after opening of valve of gas jet. Insert: Phase shift from the neutral gas jet (measured by transverse interferometry) vs. $t$.

Fig. 4.13 (main panel) shows $f_{c}(t)$ vs. time $t$ after the valve opens with an opening time of $800 \mu \mathrm{s}$ and a backing pressure of 500 psi. Each data point is the average of 20 single-shot measurements of $f_{c}$. $f_{c}$ rises between 0.3 and 0.7 ms, then levels off until $1.0 \mathrm{~ms}$ (0.2 ms after the valve closes) before dropping sharply. For comparison, the insert of Fig. 4.13 shows phase shift through the un-ionized jet measured by transverse interferometry, which is proportional to $N_{\text {tot }}(t)$. While the two curves resemble each other qualitatively, they differ in details. For example, $N_{\text {tot }}$ begins dropping after $t=0.8 \mathrm{~ms}$, whereas $f_{c}$ drops 


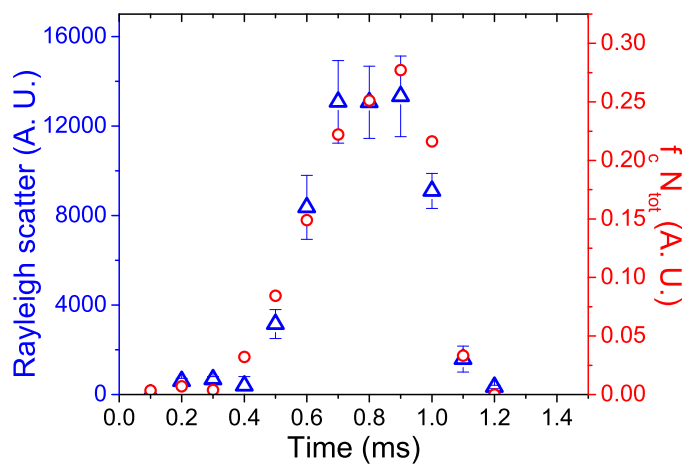

Figure 4.14: Rayleigh scatter and $f_{c} N_{\text {tot }}$ vs. time $t$.

only after $1.0 \mathrm{~ms}$. After blocking the pump beam, we measured the Rayleigh scatter, as shown in Fig. 4.14. The steady Rayleigh scatter between 0.7-0.9 ms agrees with the steady $f_{c}$ and $N_{\text {tot }}$. This time $t$ differs from the time elapsed since the atoms start to condense. The latter is $z / v$, where $z$ is the distance from the jet throat to the probed region, $v$ is the average velocity of the gas flow. Assuming $v \sim 2 \times 10^{3} \mathrm{~m} / \mathrm{s}$ [104], the corresponding time for $z=13$ $\mathrm{mm}$ is $\sim 6 \mu \mathrm{s}$, much shorter than the time $t$. Therefore, the increase of $f_{c}$ is a result of the increase in $N_{\text {tot }}$ or the equivalent pressure.

The transverse variation of $f_{c}$ with a backing pressure $P_{0}=500$ psi at $t=1.1 \mathrm{~ms}$ after the valve opens is shown in Fig. 4.15. There is a gradual drop from $2.2 \mathrm{~mm}$ to $2.9 \mathrm{~mm}$. Clusters almost vanish at $2.7 \mathrm{~mm}$ away from the jet axis where substantial monomers exist $\left(\sim 15 \%\right.$ of $N_{\text {tot }}$ at the center). In contrast, the time to reach the asymptotic phase shift shows little variation, which indicates $r_{c}$ is almost independent of the position. With an assumption 

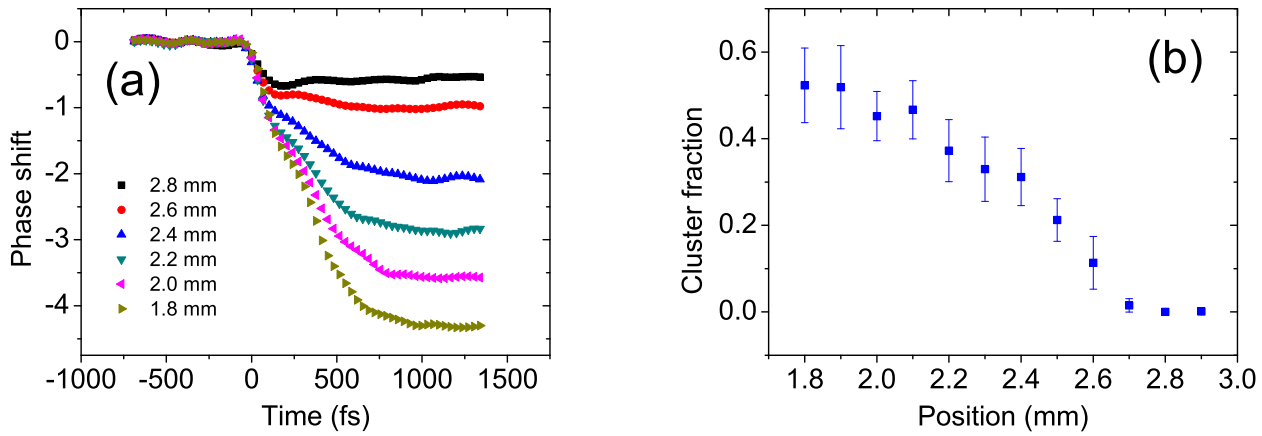

Figure 4.15: (a) Phase shifts at various position. Each curve is an average of 20 curves. (b) Cluster fraction $f_{c}$ vs. transverse position. The position represents the distance away from the center of the gas jet. The uncertainty of the center of the jet is $\sim 0.2 \mathrm{~mm}$.

of a constant $f_{c}$, a decrease of $r_{c}$ with position near the edge of the gas jet was found using Rayleigh scatter and transverse interferometry in Ref. [86]. If a constant $r_{c}$ is assumed, the same measurement can be interpreted as a decease of $f_{c}$ with position. Simulations of Ref. [94] using a liquid drop model yield a near constant $f_{c}$ and $r_{c}$ as a function of the distance from the jet axis for a three-stage nozzle. The input parameters of the simulations are different from our experiment. The discrepancy of the spatial variations of $f_{c}$ may be caused by the inadequateness of the model. The liquid droplet model may not be suited to describe small cluster nuclei using macroscopic quantities such as temperature and surface tension [94]. In addition, viscous boundary layer effects, which could be important for a diverging nozzle [105], was not included in the model. The evaporation of clusters - e.g. near the wall of the nozzle's expansion cone — was also not considered. A shock structure may appear if 
the background pressure is high [75]. The disturbance of a barrel shock can be ruled out, because the barrel shock is expected to be absent at $P_{0} / P_{b}>10^{7}$, where $P_{0}$ is the backing pressure and $P_{b}$ is the background pressure $\left(10^{-3}\right.$ Torr $)$.

The effect of probe deflection due to the atomic density gradient at the edge of the gas jet was considered. If the angle of deflection $\Delta \theta$ is small, $\Delta \theta$ can be estimated by [106]

$$
\Delta \theta=\frac{n_{\mathrm{e} 0}}{2 n_{c}} \frac{L_{y}}{L_{x}}
$$

where the gradient scale length

$$
L_{x} \equiv \frac{\int n_{e}(x, y) d y}{\frac{\partial}{\partial x} \int n_{e}(x, y) d y}=\frac{\int N_{\text {tot }}(x, y) d y}{\frac{\partial}{\partial x} \int N_{\text {tot }}(x, y) d y}=\frac{\Delta \phi_{\text {neu }}(x)}{\frac{\partial}{\partial x} \Delta \phi_{\text {neu }}(x)}
$$

and

$$
n_{e 0} L_{y}=\int n_{e}(x, y) d y=\frac{Z \Delta \phi_{\text {neu }}(x) \lambda}{(2 \pi)^{2} \alpha} .
$$

Therefore, we have

$$
\Delta \theta=\frac{Z \lambda}{2(2 \pi)^{2} \alpha n_{c}} \frac{\partial \Delta \phi_{\text {neu }}(x)}{\partial x},
$$

where $Z$ is the average charge state of the plasmas. Assuming $Z=2$ and using the density gradient in Fig 3.4 (left panel), where $\frac{\partial \Delta \phi_{\text {neu }}}{\partial x} \approx 0.8 \mathrm{rad} / \mathrm{mm}$ for $1 \mathrm{~mm}<x<2 \mathrm{~mm}$, we found $\Delta \theta=7 \times 10^{-4} \mathrm{rad}$. The phase shift caused by the deflection is estimated by $k L(\cos (\Delta \theta)-1) \approx k L(\Delta \theta)^{2}$, where $L$ is the distance to the object plane. The corresponding error of phase shift for $L=2 \mathrm{~mm}$ is $0.02 \mathrm{rad}$, much smaller than the measured phase shift. Therefore, the steering of the probe by index gradient does not affect our result.

In view of the above arguments, the most likely cause for the decrease of $f_{c}$ with radius is simply that $N_{\text {tot }}$ is also decreasing, and clusters form 
more slowly at low $N_{\text {tot }}$. An additional factor may be that cluster evaporate preferentially as the pass the wall of the nozzle's expansion cone. A dedicated simulation is required to have a full understanding.

The strong absorption and refraction near the center of the gas jet cause difficulty in getting reliable phase-shifts and identifying the contribution from monomer plasmas and clustered plasmas. Thus measurements at the center of the jet were not made. From the uniform Rayleigh scatter profile, it is believed that there is little variation in $f_{c}$ in places other than the edge. Our measurement gives an averaged $f_{c}$ along the path, which is a good estimate of the local $f_{c}$. For $f_{c}$ with cylindrical symmetry, we may determine local $f_{c}$ by Abel inversion. This can be done by finding electron density due to monomer plasmas $n_{e, m}(r)$ from $\Delta \phi_{m}(x)$ and due to cluster plasmas $n_{e, c}(r)$ from $\Delta \phi_{c}(x)$. Other approaches for determining local $f_{c}$ include a non-collinear pump-probe configuration or focusing the pump to a Rayleigh length much shorter than the jet thickness. However, the phase shifts in both situations are contributed from monomer plasmas and clustered plasmas of various ionization degree, thus complicating the extraction of $f_{c}$ from the phase shifts.

The spatial variations of $f_{c}$ at several pressures are shown in Fig. 4.16(a). The dependence of $f_{c}$ on the pressure at $x=1.5 \mathrm{~mm}$ is given in Fig. 4.16(b). For $P<400$ psi, $f_{c}$ increases monotonically with the pressure. A saturation of $f_{c}$ appears after 400 psi.

To measure the cluster fraction at low temperature, we cryogenically cooled a series-99 valve using the cooling system described in Chapter 3 . The 

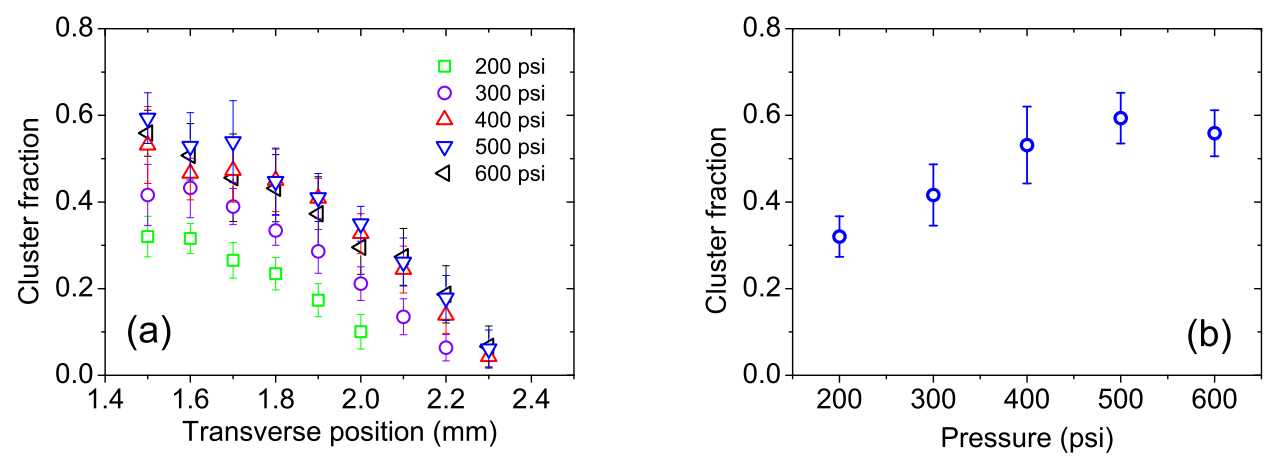

Figure 4.16: (a) Cluster fraction $f_{c}$ vs. position for several different pressures measured at $1 \mathrm{~ms}$ after the valve opens. The opening time of the valve is 1.2 ms. $f_{c}$ was extracted from each shot. Each data point is 20 shots average. The error bar is the standard deviation. (b) Cluster fraction $f_{c}$ vs. pressure for $x=1.5 \mathrm{~mm}$.

system required considerable time to stabilize the temperature. For convenience, we cooled the jet down to $170 \mathrm{~K}$ and then let the temperature increase slowly. The change of temperature during the time for acquisition of 40 shots was within $1 \mathrm{~K}$. Cluster fraction $f_{c}$ vs. temperature is shown in Fig. 4.17. The backing pressure was $240 \mathrm{psi}$, so even at $170 \mathrm{~K}$, the phase shift reaches the asymptotic value before $1.5 \mathrm{ps}$. We may increase $I_{\mathrm{pu}}$ to reduce the expansion time. A different nozzle (500 $\mu \mathrm{m}$ orifice, $5^{\circ}$ half angle) was used. As the temperature decreases, the cluster size increases, which is seen from the time needed for the phase shifts to reach the asymptotic value. However, no significant enhancement of the cluster fraction was observed. Temperature dependence may be different at high pressure. 

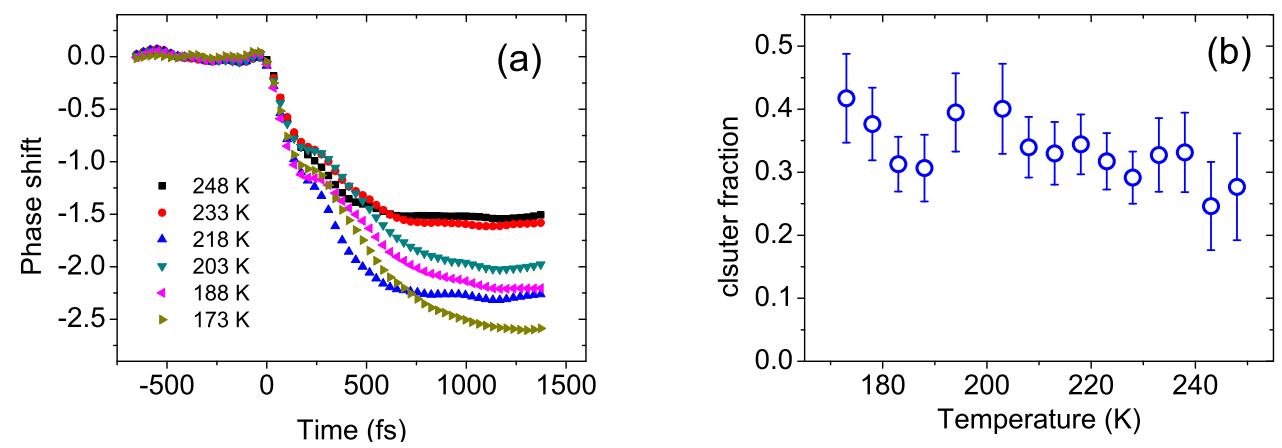

Figure 4.17: (a) Measured phase shifts at various temperature. Each curve is an average of 40 curves. (b) Cluster fraction $f_{c}$ vs. temperature. $f_{c}$ was extracted from each shot. The result is 40 shots average. The error bar is the standard deviation.

\subsubsection{Discussion}

For an accurate extraction of $f_{c}$ from the measurement, accurate $Z_{m}$ and $Z_{c}$ are essential. This is trivial for hydrogen clusters. $Z_{c}=1$ and $Z_{m}=1$ can be guaranteed if $I_{\mathrm{pu}}>1.4 \times 10^{14} \mathrm{~W} / \mathrm{cm}^{2}$. For argon clusters irradiated at moderate intensity, our $Z_{c}$ was not verified in an independent experiment. In Chapter 2, we showed that our model gives similar results to that from a PIC simulation. The validity of the model can be checked indirectly from the dependence of $Z_{c}$ on the laser intensity. Fig. 4.18 shows reconstructed single-shot phase shifts vs. time and position. $\Delta \phi_{m}$ and $\Delta \phi_{c}$ were extracted from lineout at each position and plotted in Fig. 4.19. Assuming a Gaussian spatial distribution with peak intensity of $4 \times 10^{14} \mathrm{~W} / \mathrm{cm}^{2}$, we calculated $Z_{m}$ and $Z_{c}$ at each position using the corresponding intensity. The spot size $w_{0}$ is chosen as $42 \mu \mathrm{m}$ to fit $\phi_{m}$. We compared the measured phase shifts to the 


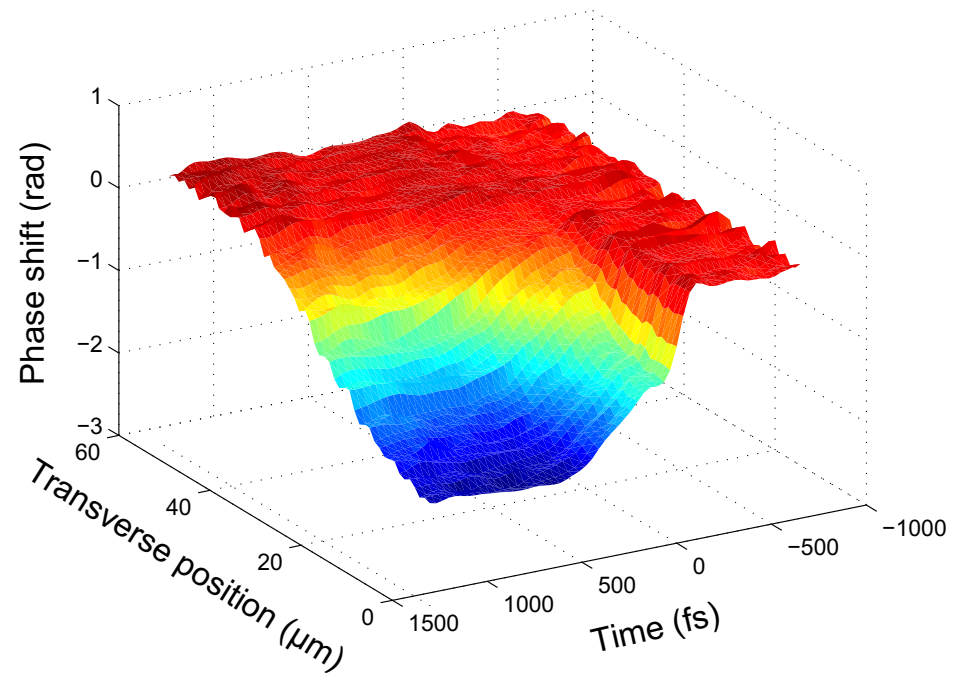

Figure 4.18: Perspective view of the time-resolved phase shifts.

computed phase shifts ${ }^{3}$ using $f_{c}=0.26$. Except for a considerable discrepancy at low intensity $\left(I_{\mathrm{pu}}<2.0 \times 10^{14} \mathrm{~W} / \mathrm{cm}^{2}\right.$, positioned at $\left.-25 \mu \mathrm{m}\right)$, of which the cause is discussed in Chapter 2 (p. 22), both of the computed and measured phase shifts show a similar weak dependence on $I_{\mathrm{pu}}$.

We may determine $Z_{c}$ using the phase shift from a neutral gas jet $\Delta \phi_{\text {neu }}$ and the phase shift from the monomer plasmas $\Delta \phi_{m}$ from the same position of the gas jet. The phase shift from a neutral gas jet is

$$
\Delta \phi_{\text {neu }}=\frac{(2 \pi)^{2} \alpha_{\text {neu }}}{\lambda_{\text {neu }}} \int N_{\text {tot }}(z) d z .
$$

Combining Eqs. 4.4 and 4.13, we have

$$
\frac{\lambda_{\mathrm{FDH}} \Delta \phi_{m}}{\lambda_{\text {neu }} \Delta \phi_{\text {neu }}}=\frac{Z_{m}\left(1-f_{c}\right) / n_{\text {crit }}+4 \pi \alpha_{\mathrm{FDH}}}{4 \pi \alpha_{\text {neu }}} .
$$

\footnotetext{
${ }^{3}$ For simplicity, the atomic polarizability is not considered here, i.e., $\Delta \phi_{m} \propto Z_{m}$.
} 


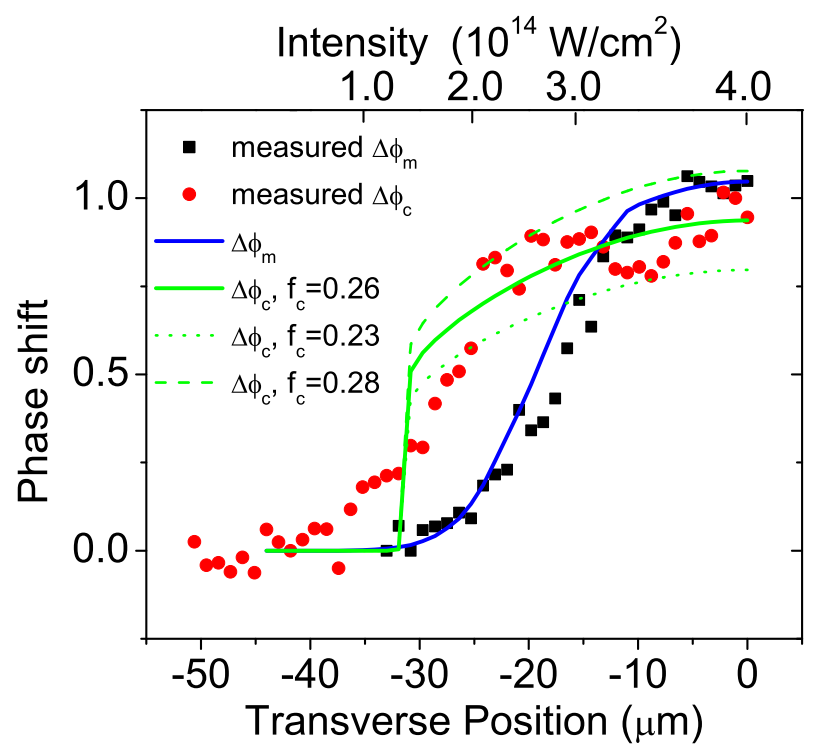

Figure 4.19: Measured and calculated phase shifts vs. position.

Therefore, we may find $f_{c}$ without the knowledge of $Z_{c}$. Once $f_{c}$ is found, $Z_{c}$ can be determined using Eq. 4.6. However, current setup does not allow us to measure $\Delta \phi_{\text {neu }}$ and $\Delta \phi_{m}$ at the same position of the gas jet. Using $\Delta \phi_{\text {neu }}$ extracted from a transverse interferometry measurement, the resulted $Z_{c}$ is much smaller than $Z_{m}$. This incorrect $Z_{c}$ may be caused by the large uncertainty in determining $\Delta \phi_{\text {neu }}$.

The red squares in Fig. 4.20 are measured phase shifts. For the conditions of the red squares, we found $r_{c}=6 \pm 1 \mathrm{~nm}$ using Rayleigh scatter [86], transverse interferometry, and the knowledge of $f_{c}$. The blue line is a simulated $\Delta n$ contributed by expanding clusters with an initial cluster size of 7 $\mathrm{nm}$. The consistency of the expansion time is another support of our model.

Although $N_{\text {tot }}$ measurement shows little shot-to-shot fluctuation, the 


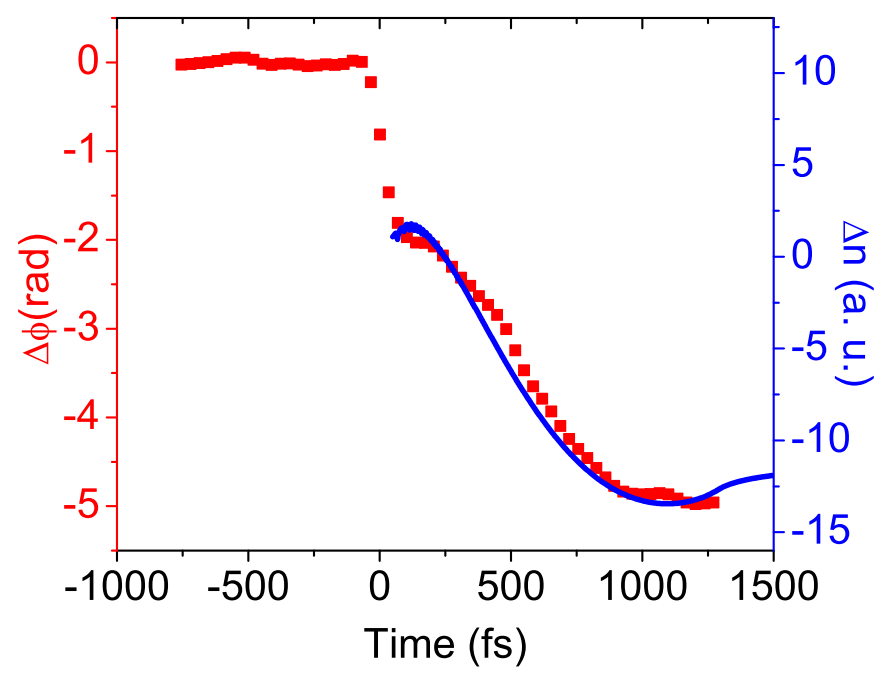

Figure 4.20: Measured phase shifts (red squares) and simulated cluster contribution to the refractive index (blue line)

Rayleigh scattering profile shows considerable fluctuation, indicating the shotto-shot fluctuation of $f_{c}$ and/or $r_{c}$. To quantify fluctuation of $f_{c}$ from the standard deviation of our measurement is premature. Errors in $f_{c}$ may come from the fluctuation of the laser intensity or errors in the reconstructed phase shifts. The pump intensity at the region sampled by the entrance slit of the spectrometer may vary quite a lot because of the limited pointing stability and the energy fluctuation of the laser. The signal-to-noise ratio also affects the reconstructed phase shifts. An error of $\sim 100 \mathrm{mrad}$ is expected if we use the full dynamic range of a 12 bit CCD. In Fig. 4.18 where the result is of good quality, the maximum phase shift is $\sim 2 \mathrm{rad}$. For small phase shifts, the signal may be buried by the noise. For large phase shifts, the refraction and 
absorption worsen the result and two steps cannot be clearly identified.

The initial drop ends at $t \sim 100$ fs. Any positive contribution to the refractive index from the clustered plasma at that time is neglected. This is acceptable because even the peak of the positive contribution is $<10 \%$ of the asymptotic negative contribution. If $f_{c}$ is close to 1 , however, ignoring the positive contribution, which is no longer small compared with the contribution from monomer plasmas, will lead to substantial error in the extracted $f_{c}$.

Our method is well suited for experiments with multi-terawatt fs laser systems, since only a small fraction of the typical pulse energy $(\geq 1 \mathrm{~J})$ of such systems need be split off to make the required pump and probe pulses. Indeed, an in situ measurement with the main experiment is preferred since the prepulse may modify gas jet properties [107]. In such a case, we would expect that cluster sizes and cluster fraction reach a minimum on the laser axis, where the intensity peaks. For current experiments, we do not expect the influence of prepulse owing to our moderate $I_{\mathrm{pu}}$ and high contrast ratio.

It is worth pointing out that our measurements and the simulations of Ref. [94] show that the majority of atoms are unclustered. At $P>400$ psi, $f_{c}$ as high as 0.6 was found. A direct quantitative comparison with the results of the model of Ref. [94] would be useful, but it requires a dedicated simulation that uses our nozzle geometry. 


\subsection{Cluster-size distribution}

We determined the cluster mass fraction from the asymptotic value of the phase shift. The cluster size can be estimated from the expansion time, as shown in Fig. 4.20. The expansion dynamics contains additional information of the clusters. The cluster-size distribution can be recovered from the fstime-resolved absorption and phase shift measurements if a forward model that maps the size distribution to the measured data is given [50].

The time-dependent refractive index after laser irradiation is

$$
\tilde{n}(\tau)-n_{\text {gas }}=-\frac{1}{2} \frac{\omega_{m}^{2}}{\omega_{\mathrm{pr}}^{2}}+2 \pi \int_{0}^{\infty} \alpha\left(\tau, R_{0}\right) F\left(R_{0}\right) \mathrm{d} R_{0},
$$

where $\tilde{n}(\tau)=n(\tau)+\mathrm{i} \eta(\tau)$ is the complex refractive index, $\alpha\left(\tau, R_{0}\right)$ is the polarizability of the expanding cluster of initial radius $R_{0}, F\left(R_{0}\right)$ is the size distribution, and $F\left(R_{0}\right) \mathrm{d} R_{0}$ is the number of clusters per unit volume with initial radii ranging from $R_{0}$ to $R_{0}+\mathrm{d} R_{0}$.

The measured absorption $A(\tau)$ and phase shift $\Delta \phi(\tau)$ are related to the refractive index by

$$
\begin{aligned}
A(\tau) & =1-\exp \left(-2 \frac{\omega_{\mathrm{pr}} L}{c} \eta(\tau)\right), \\
\Delta \phi(\tau) & \left.=\frac{\omega_{\mathrm{pr}} L}{c}\left(n(\tau)-n_{\text {gas }}\right)\right),
\end{aligned}
$$

where $L$ is the effective interaction length. The measured data are related to 
the size distribution $F\left(R_{0}\right)$ by

$$
\begin{aligned}
\ln (1-A(\tau)) & =-\frac{8 \pi^{2}}{\lambda} \int_{0}^{\infty} \operatorname{Im}\left[\alpha\left(\tau, R_{0}\right)\right] F\left(R_{0}\right) L \mathrm{~d} R_{0} \\
\Delta \phi(\tau)-\Delta \phi_{m} & =\frac{4 \pi^{2}}{\lambda} \int_{0}^{\infty} \operatorname{Re}\left[\alpha\left(\tau, R_{0}\right)\right] F\left(R_{0}\right) L \mathrm{~d} R_{0} .
\end{aligned}
$$

Eq. 4.18 and 4.19 are Fredholm integral equations of the first kind, which are ill-posed problems. To simplify the problems, we may add a priori information that clusters follow a log-normal size distribution [88, 108]. The log-normal distribution may be disturbed, for example, when a prepulse is strong enough to destroy the clusters partially. A regularization method is then needed; it is required to have sufficiently small measurement noises or model errors to find a reliable solution of the problem.

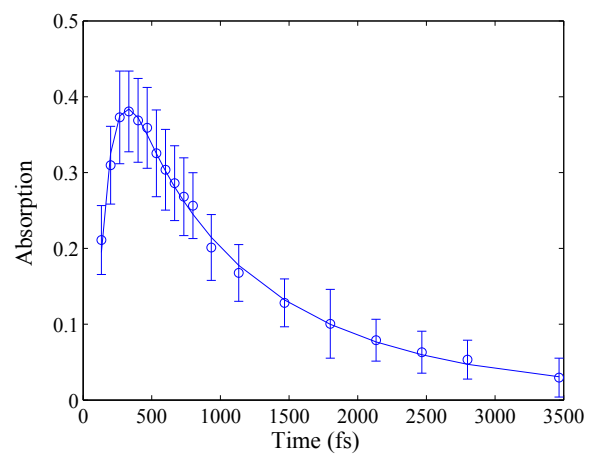

Figure 4.21: Measured (open circles) and calculated (solid line) absorption $A(\tau)$ as a function of the delay $\tau$.

Fig. 4.21 shows the measured and calculated absorption as a function of the delay. To achieve a good fit of the absorption data, we used a shifted 
log-normal distribution,

$$
F\left(R_{0}\right)=\frac{c}{4 \pi \omega} \frac{1}{L a^{4}} \frac{B}{\sigma \sqrt{2 \pi}\left(R_{0} / a-\chi\right)} \exp \left(-\frac{\left[\ln \left(R_{0} / a-\chi\right)-\mu\right]^{2}}{2 \sigma^{2}}\right),
$$

where $B=0.182, \chi=0.27, \mu=-2.33, \sigma=0.93$. The shift $\chi$ was non-zero probably because the model is not adequate to describe $\alpha\left(\tau, R_{0}\right)$ of small clusters. The model used in this fitting did not include three-body recombination. Details of the measurement and model can be found in Ref. [50].

Simultaneous measurements of the absorption and phase shift using FDH are helpful since we may crosscheck the result if a size distribution is extracted. To recover a reliable cluster-size distribution, effects to redue the errors in the measurements are needed. For the absorption data in Fig. 4.9, we noticed the presence of large errors for $t>1$ ps. Indeed, the absorption tail is critical for recovering the distribution of large clusters, since the absorption at longer time are contributed by large clusters. A pump-probe multi-shot measurements with a variable delay can give reliable absorption at a longer delay. We presented an absorption measurement in Appendix A. $I_{\text {pu }}$ should be chosen at which our model is valid. We need to consider the spatial variation of $I_{\mathrm{pu}}$ when the spot size of the probe and probe are comparable. 


\section{Chapter 5}

\section{Harmonic generation in cluster jets}

\section{$5.1 \quad$ Introduction}

Studies in low-order harmonics are driven by several motivations. First, the harmonics are of potential for developing a powerful ultrashort ultraviolet source [109]. Second, they can be used to test new phase-matching or quasi-phasing-matching schemes that may be scalable to HHG [29]. Third, they can be an effective diagnostics; for example, third harmonic generation microscopy [110] has been a standard technique.

Several pump-probe type experiments demonstrated enhancement of third harmonic generation (THG) at very different time scales. Enhancement appearing $\mu$ s after a pump pulse is attributed to excited-state neutrals [111]. Enhancement during filamentation [112] or immediately after the creation of plasmas [113] is attributed to an enhanced third order susceptibility $\chi^{(3)}$ of monomer plasmas [114] and/or propagation effects [115]. At sub-ps, a transient THG enhancement was observed by Shim et al. in cluster jets for a pulse delayed $\sim 300$ fs with respect to a $10^{15} \mathrm{~W} / \mathrm{cm}^{2}$ ionizing pulse [29]. The enhancement is originated from the resonantly enhanced $\chi^{(3)}$ of clustered plasmas, and partial phase-matching owing to the positive contribution of clustered 
plasmas to the refractive index. Moreover, the enhancement exhibits strong polarization anisotropy attributed to the angular dependence of the ion density nonuniformity [116], allowing one to probe early cluster expansion dynamics, particularly its anisotropy. In the above experiment, harmonics were detected by a PMT in the air with wavelength discriminated by a spectrometer and a $265 \mathrm{~nm}$ bandpass filter. Detection of higher-order harmonic is not possible since air absorbs light at $\lambda<200 \mathrm{~nm}$. In addition, the PMT may not collect all the third-harmonic radiation if strong frequency shifts occur. Aiming at extending the enhancement to high-order harmonics, we continue the study of enhanced harmonic generation by using a vacuum spectrometer.

\section{$5.2 \quad$ Experimental setup}

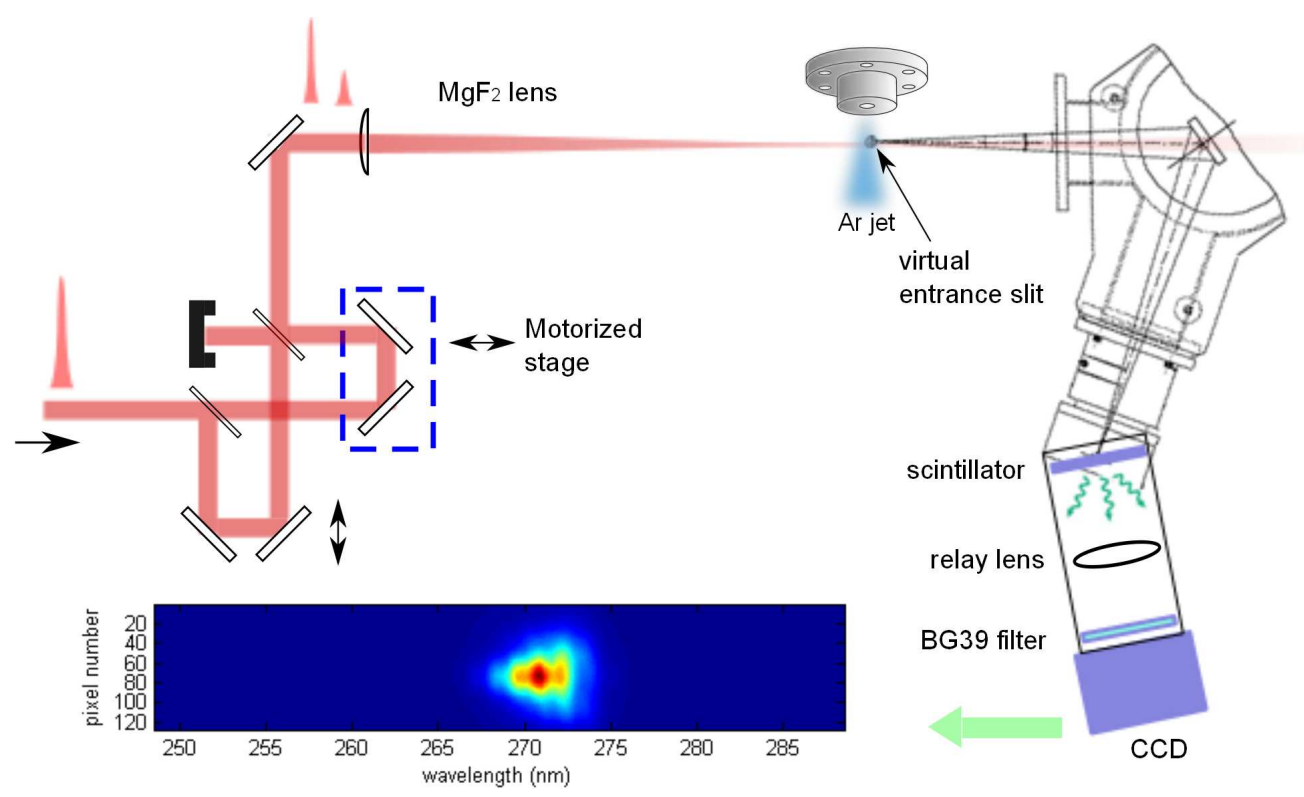

Figure 5.1: Setup for harmonic detection 
Fig. 5.1 shows the schematic of harmonic detection system. Harmonics were detected by a vacuum ultra-violet Seya-Namioka monochromator (McPherson M235 M3) with a 500-mm focal length, platinum coated, 1200 $\mathrm{G} / \mathrm{mm}$ concave grating blazed at $70 \mathrm{~nm}$. Due to astigmatism, the image height at the image plane is approximately $2 / 3$ of the illuminated grating height for a small size source. A sodium salicylate plate located at the image plane converts VUV light into visible light with a quantum efficiency typically exceeding 0.6. The visible light was relay-imaged to a TE-cooled CCD (Andor DU401A-BV, $1024 \times 127$ pixels, $26 \times 26 \mu \mathrm{m}^{2}$ ) where dispersion is $0.04 \mathrm{~nm} /$ pixel. The stray light from the fundamental was blocked by a BG39 filter. Based on the geometry and angular distribution of fluorescence [117], we estimated that $\sim 4 \%$ of the fluorescence was collected by the lens.
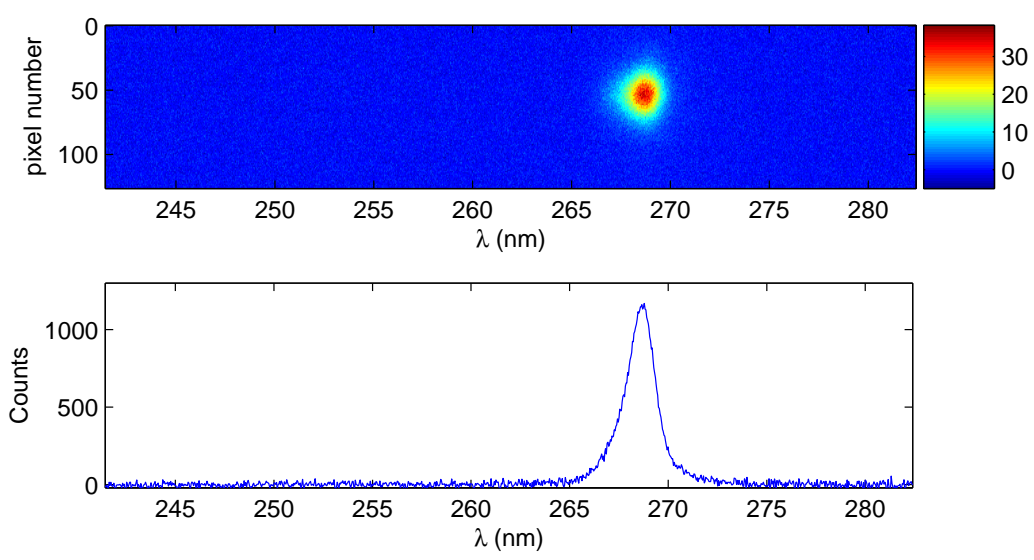

Figure 5.2: Third harmonic spectrum (1 shot)

We tested the monochromator using harmonics from the gas jet irradiated by 40 fs pulses of $\sim 1 \mathrm{~mJ}$. The jet was positioned in front of the laser 

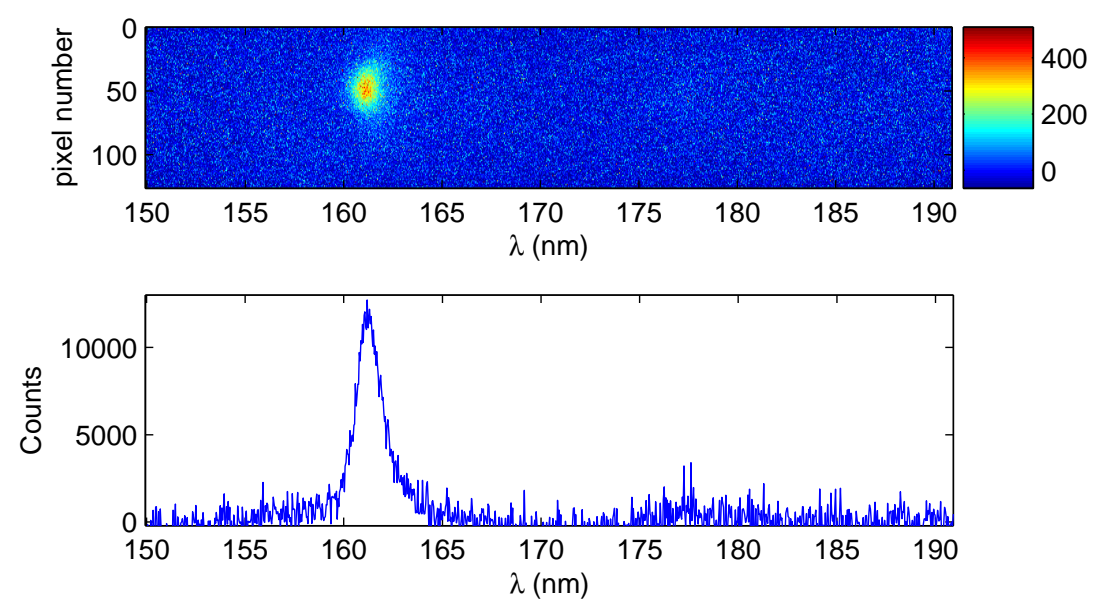

Figure 5.3: Fifth harmonic spectrum $\left(10^{4}\right.$ shots $)$

focus. Fig. 5.2 and 5.3 show the third and fifth harmonics, respectively. The ratio of 3rd and 5th harmonic energy is on the order of $10^{-3}$, consistent with reported measurements [118]. A ratio of $3 \times 10^{-4}$ was predicted for $\lambda=1330 \mathrm{~nm}$ using the standard Kerr effect model [119].

\subsection{Enhanced third-harmonic generation in cluster jets}

As a demonstration of enhanced harmonic generation from expanding clusters, an experiment using two collinear pulses of the same color was performed. Two pulses were created from a 7:3 beamsplitter and recombined on a 50/50 beamsplitter, as shown in Fig. 5.1. We found the zero delay by observing the spatial interference while manually adjusting a translation stage. The accuracy of the zero delay is within $20 \mathrm{fs}$, which was checked by autocorrelation of two pulses passing through a $1 \mathrm{~mm}$ KDP crystal. The second harmonic 
generation of this autocorrelation is shown in Fig. 5.4. A computer controlled delay line enabled us to perform a programmed scan. The pulses were focused by a $2^{\prime \prime} \mathrm{MgF}_{2}(f=68.7 \mathrm{~cm}$ for $800 \mathrm{~nm})$ singlet lens. We apertured the beam to adjust the intensity of the pulse at the focus where interaction occurred. The leading pulse served as the pump. The trailing pulse excited harmonics from the clustered plasma. Pump and probe interfered when they were overlapped in time. Nevertheless, the interference did not affect our desired enhancement that occurred $\sim 200$ fs after the pump.

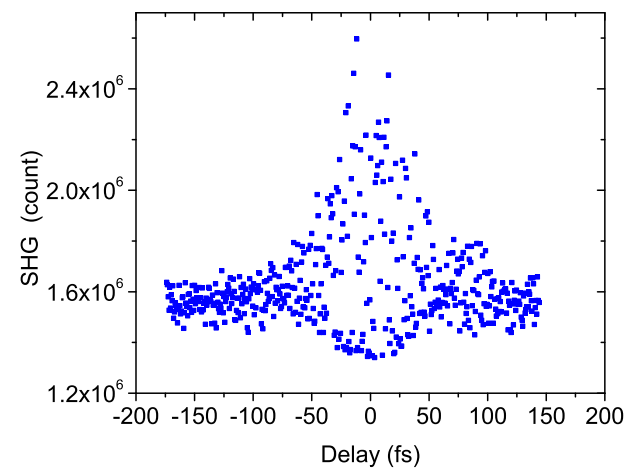

Figure 5.4: Autocorrelation of pump and probe to check zero delay. The result is shifted by -15 fs to have it center at time zero.

Fig. 5.5(a) shows THG from the center of gas jet with a backing pressure of 500 psi. The intensity of the weak pulse was $\sim 1.5 \times 10^{14} \mathrm{~W} / \mathrm{cm}^{2}$, which was estimated from the plasma emission. The intensity of the strong pulse was $\sim 3 \times 10^{14} \mathrm{~W} / \mathrm{cm}^{2}$. THG was contributed by both pulses. Their contributions can be separated except for the time when two pulses are overlapped in time. The contribution from the strong pulse is shown in Fig. 5.5(b). The corre- 

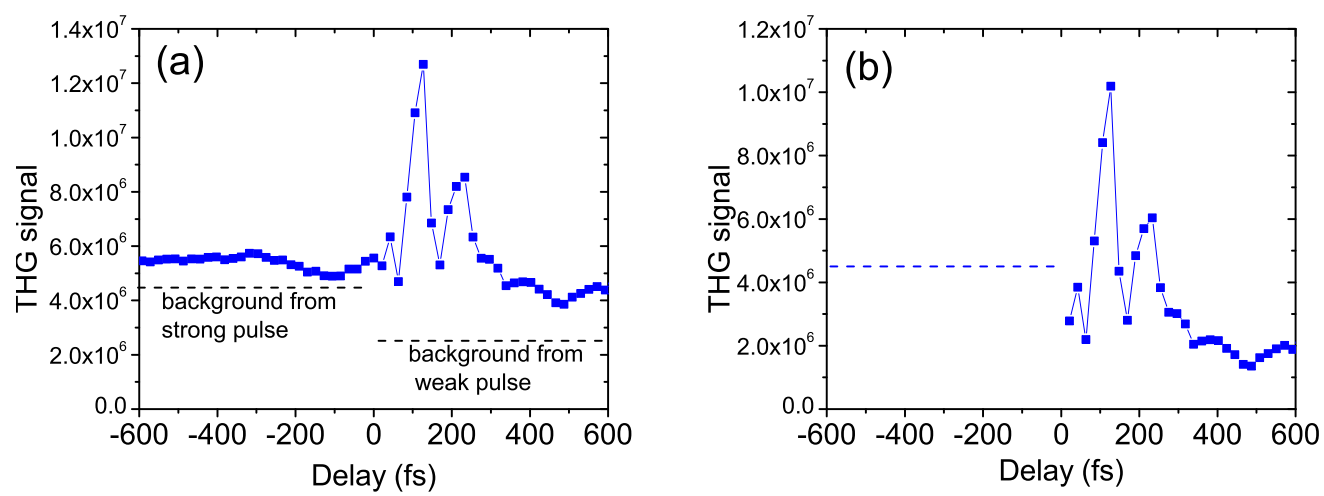

Figure 5.5: (a) THG signal. For each data point, the CCD was exposed for 100 shots (b) Contribution from the strong pulse.

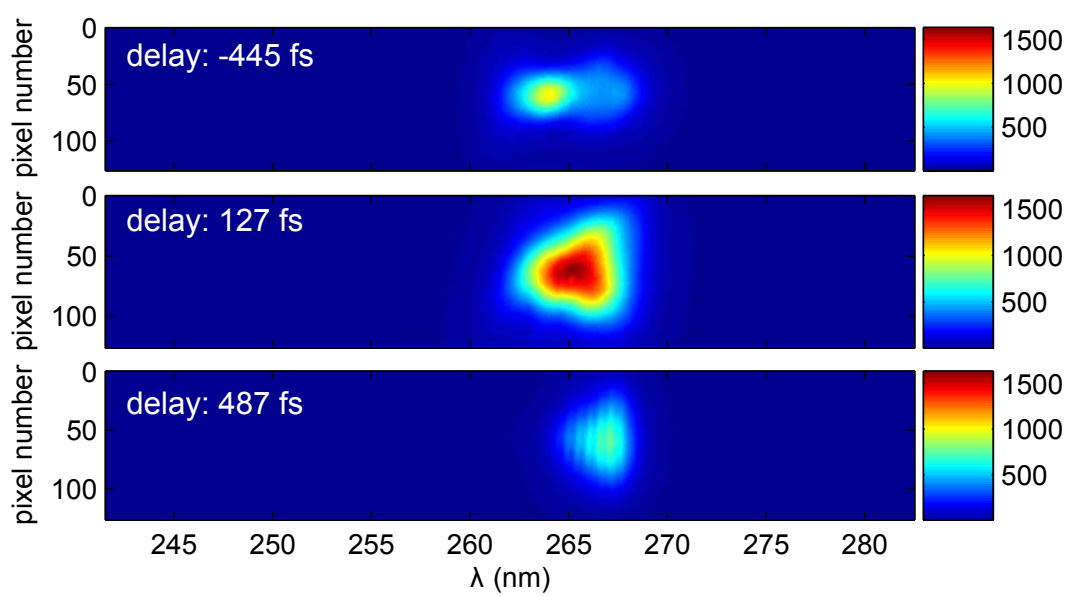

Figure 5.6: Third harmonic spectra at three different delays. The CCD was exposed for 100 shots.

sponding spectra at three delays are shown in Fig. 5.6. Shim et al. measured THG at the center of the gas jet and found that THG was suppressed after a delay of $\sim 200$ fs because of the strong absorption of the fundamental [120]. We also observed a reduction of THG at $t \sim-100 \mathrm{fs}$. For $t>0$, due to the 
weak ionization of the clusters, the absorption is not substantial, and an enhancement is observed. To excite THG from an individual clustered plasma efficiently, a probe intensity exceeding $10^{15} \mathrm{~W} / \mathrm{cm}^{2}$ is usually required. At our probe intensity, phase-matching may play a major role in the enhancement. Since the phase-matching is determined by the linear property of the jet which is expected to be isotropic, a study of the polarization dependence will allow us to determine whether phase-matching or resonantly enhanced $\chi^{(3)}$ is the main contribution of the enhancement. However, the polarization dependence of the beamsplitters in current setup prevents us from further investigation.

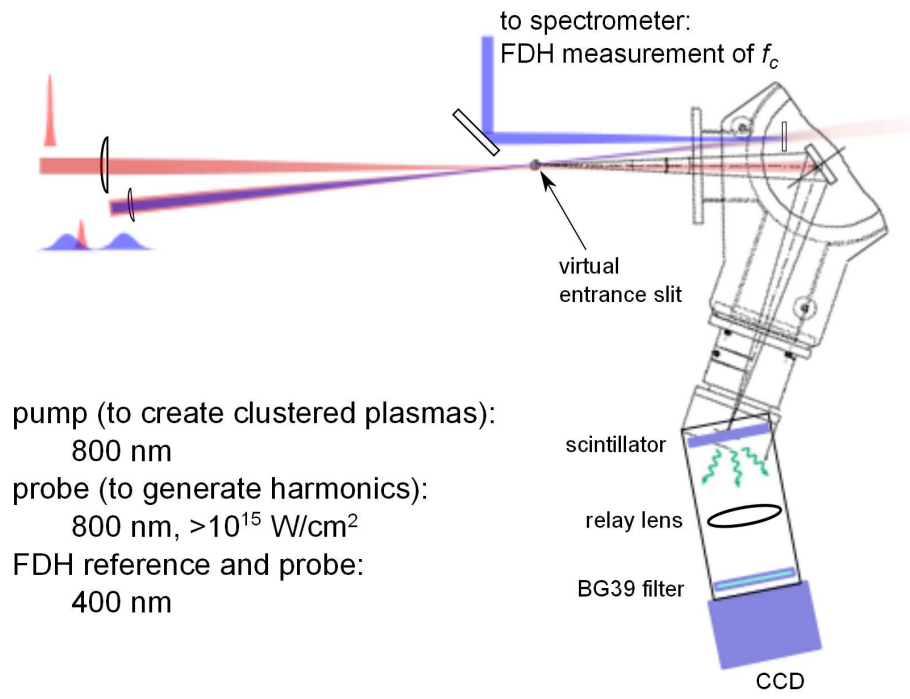

Figure 5.7: Non-collinear pump-probe schematic.

Fig. 5.7 shows a planned setup. A non-collinear configuration would have several advantages. Our $f_{c}$ measurement shows that $f_{c}$ is low near the edge of the gas jet, where experiments of Shim et al. [29] were performed. A 
non-collinear pump-probe allows the probe to interact with clustered plasmas only at the pumped region at the center of the jet. A FDH experiment may be performed at the same time, permitting real-time in situ monitoring of the phase-matching condition. 


\section{Chapter 6}

\section{Conclusions and outlook}

We present an in-depth study of a rapid, noninvasive, single-shot optical method of determining cluster mass fraction $f_{c}(\boldsymbol{r}, t)$ at specified positions $\boldsymbol{r}$ within, and at time $t$ after opening the valve of, a high-pressure pulsed supersonic argon jet. A fs pump pulse singly ionizes monomers, while quasistatically ionizing and heating clusters to a level at which recombination remains negligible as clusters expand. Under these conditions, index evolves in two simple steps corresponding to monomer and cluster contributions, allowing recovery of $f_{c}$ without detailed cluster dynamic modeling. If a high $f_{c}$ is desired, the laser-gas interaction region should be placed near the center of the gas jet with sufficient time after the valve opens.

Optical FDH has proven to be an effective tool for measuring ps scale dynamics with fs resolution in a single shot. By exploiting the $2 \mathrm{D}$ nature of the hologram (frequency and spatial dimension), we may study intensitydependent dynamics in a single shot. This technique may be used to study cluster dynamics after the cluster is irradiated by XUV pulses, which is of

recent interest [121]. One may also be able to observe the transition between Coulomb explosion and hydrodynamic expansion as $I_{\mathrm{pu}}$ varies. 


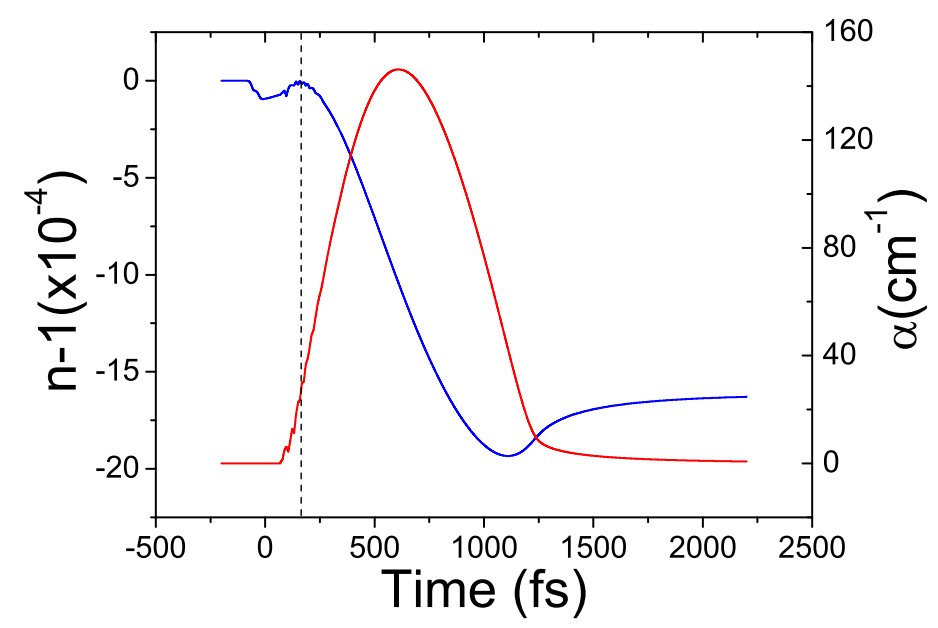

Figure 6.1: The refractive index (blue) and absorption coefficient (red) for a argon jet of $N_{\text {tot }}=10^{18} \mathrm{~cm}^{-3}, f_{c}=0.8, r_{c}=20 \mathrm{~nm}$. The jet is irradiated by $I_{\mathrm{pu}}=10^{15} \mathrm{~W} / \mathrm{cm}^{2}, 100 \mathrm{fs}, 400 \mathrm{~nm}$ pulses

Conditions for $f_{c}>0.5$ are found in experiments. To have $L_{\mathrm{coh}}=\infty$, $f_{c}=0.8$ is expected for $I_{\mathrm{pu}}=10^{15} \mathrm{~W} / \mathrm{cm}^{2}$, as shown in Fig. 6.1. For $L_{\text {coh }}$ not being the limiting factor, the requirement of $L_{\mathrm{coh}}$ may be relaxed depending on the absorption lengths of the fundamental and harmonics. The absorption length of the fundamental in Fig 6.1 is $\sim 0.4 \mathrm{~mm}$. The absorption length of the harmonics is difficult to estimate. At $100.8 \mathrm{~nm}$, the average absorption cross section per atom in a cluster was estimated to be 24.1 Mbarn from the experiment, while the estimate from simulations is 5.8 Mbarn [122]. At $\lambda=$ $72.7 \mathrm{~nm}$, the absorption cross section for a monomer argon is 33 Mbarn [123], which corresponds to absorption length of $3 \mathrm{~mm}$ for a gas of $10^{18} \mathrm{~cm}^{-3}$. The absorption cross section for atoms in clustered plasmas would be smaller since the atoms are pre-ionized. The cluster fraction of other gases such as Xe or 
gas mixture is worth investigating. Even without a perfect phase-matching, an increase in $f_{c}$ increases $L_{\text {coh }}$, thus improving conversion efficiency. While other phase-matching or quasi-phase-matching schemes are limited to a moderate laser intensity $\left(I \lesssim 10^{15} \mathrm{~W} / \mathrm{cm}^{2}\right)$, the phase-matching scheme using clustered plasmas can be used at higher laser intensity, which is critical for extending the cutoff of HHG. 
Appendices 


\section{Appendix A}

\section{Pump-probe absorption measurements}

Expanding clusters exhibit time-dependent absorption. This property can be used to find the size distribution [50]. Here the absorption of $400 \mathrm{~nm}$ light by clusters heated by $800 \mathrm{~nm}$ pump pulses was measured. The setup is shown in Fig. A.1. The pump and probe propagated collinearly through the center of the gas jet. A computer controlled delay line varied the delay between the pump and the probe. Two photodiodes recorded the energy of a transmitted pulse and a reference pulse, respectively.

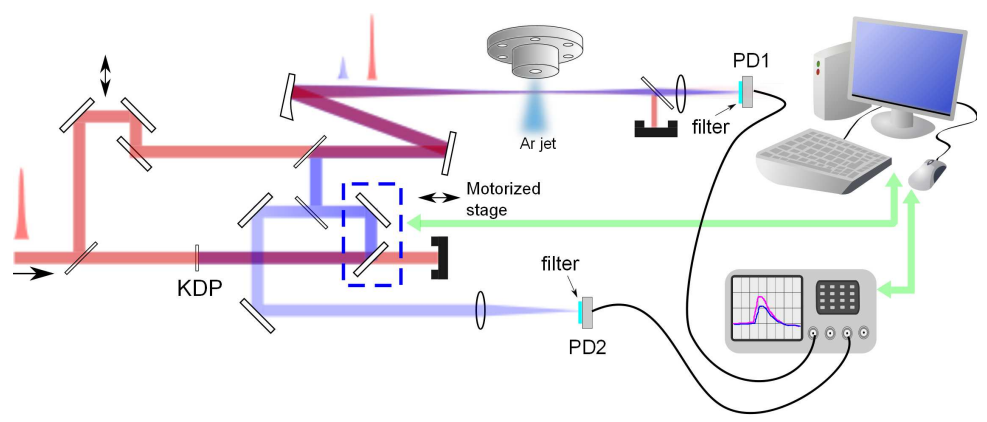

Figure A.1: Setup for absorption measurement.

Fig. A.2 shows the absorption in the cluster jet with a conical nozzle $\left(d=750 \mu \mathrm{m}\right.$ and $\left.\alpha=11^{\circ}\right)$ at $I_{\mathrm{pu}} \sim 1.5 \times 10^{14} \mathrm{~W} / \mathrm{cm}^{2}$. The backing pressure was 500 psi. Each data point is 100 shots average. Based on the discussion 
on p. 22 and p. 66, the current model does not work well at $I_{\mathrm{pu}} \sim 1.5 \times$ $10^{14} \mathrm{~W} / \mathrm{cm}^{2}$. Thus attempts to recover the size distribution were not made.

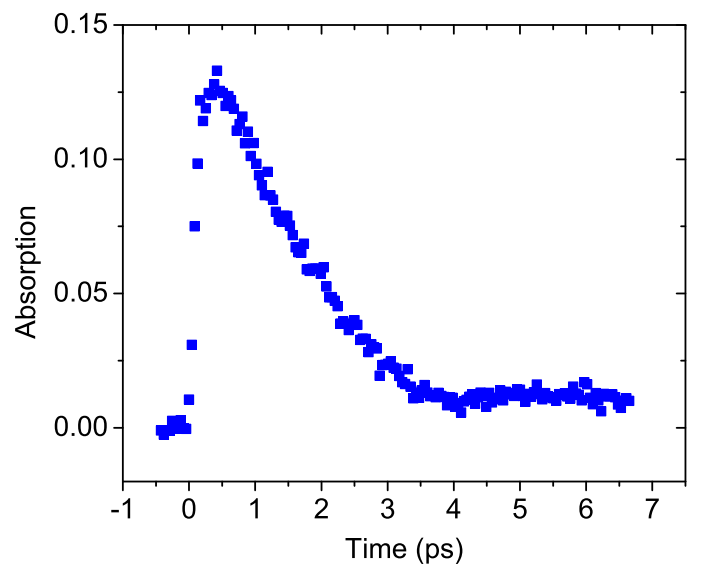

Figure A.2: Absorption measurement 


\section{Appendix B}

\section{$\mathrm{C}++$ code for ionization/heating of the cluster}

This program includes a main file, main.cpp, and a header file, cluster.h. An ODE solver in GNU Scientific Library [124] was used. The main file, main.cpp, is listed here.

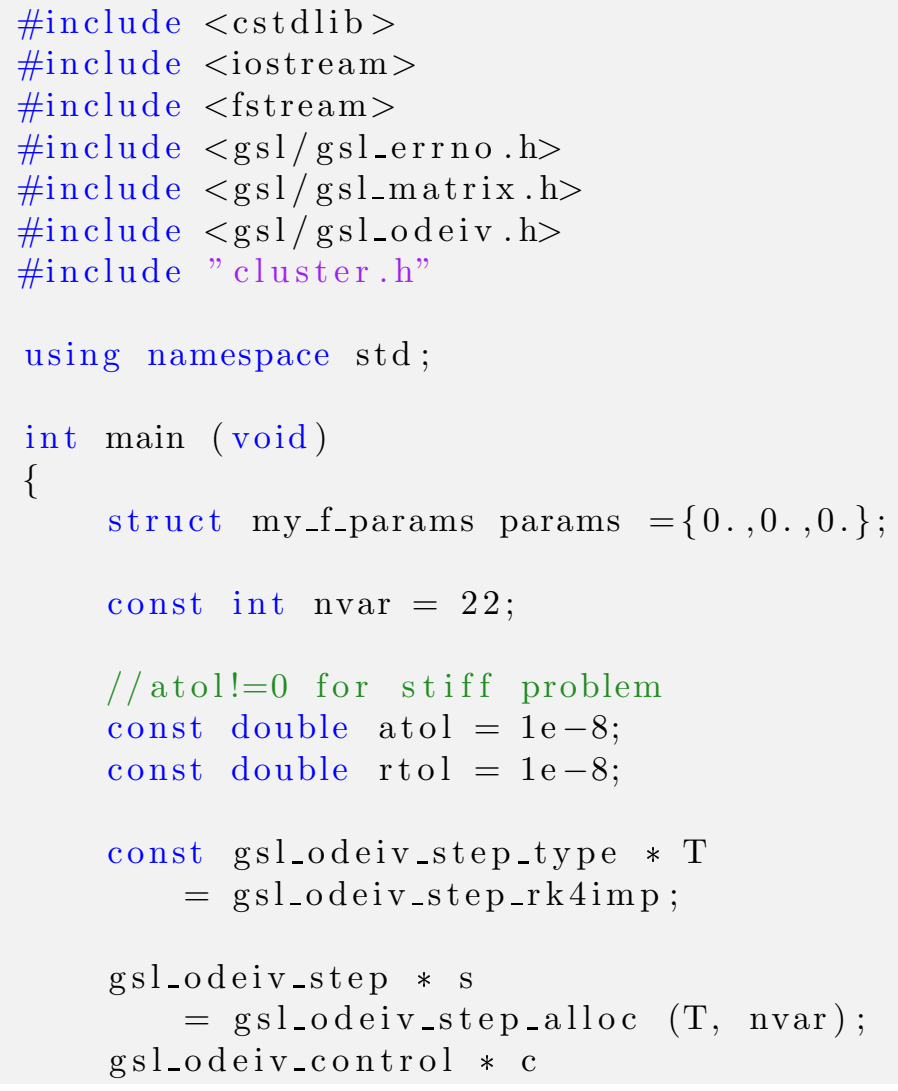




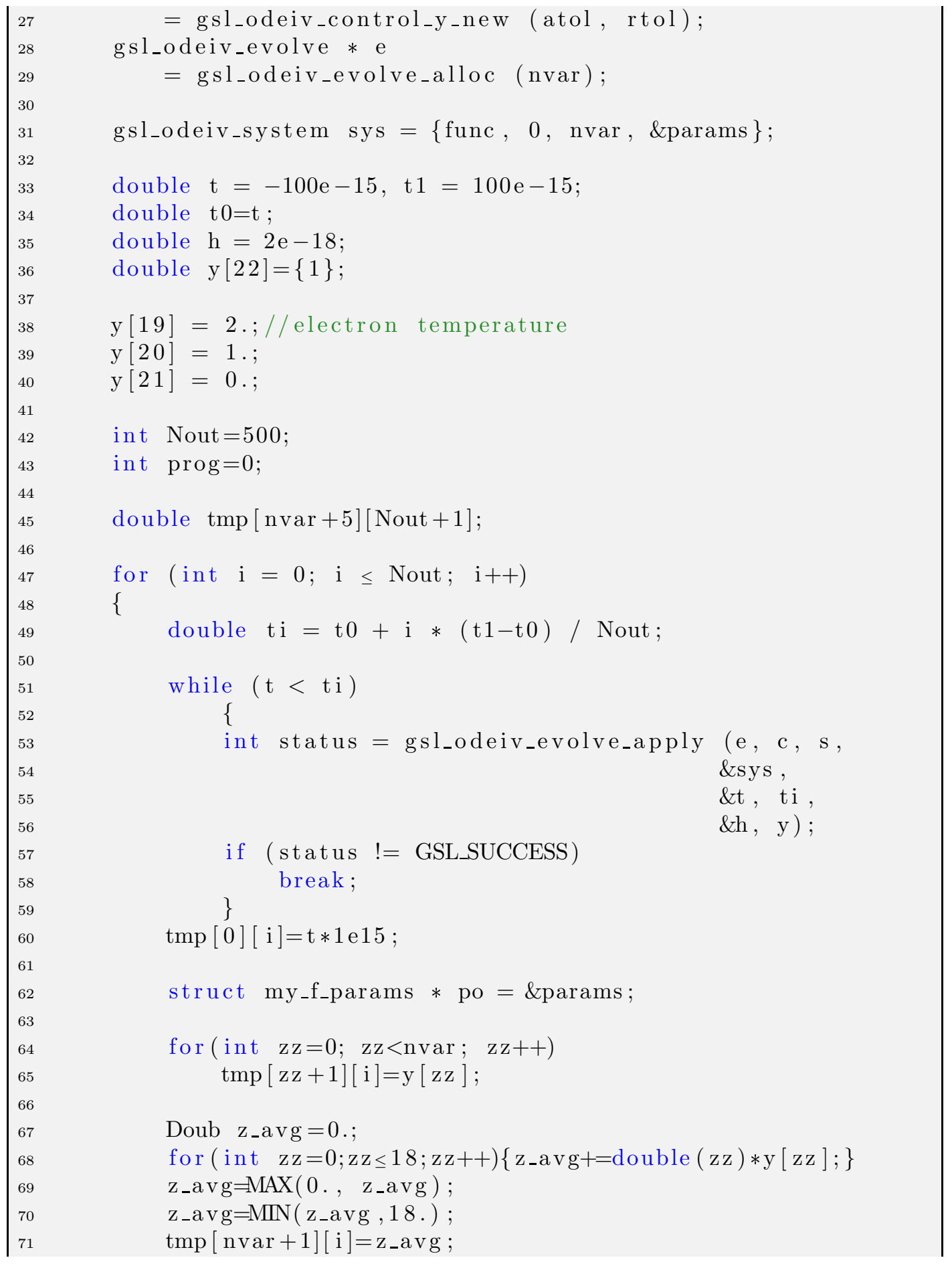




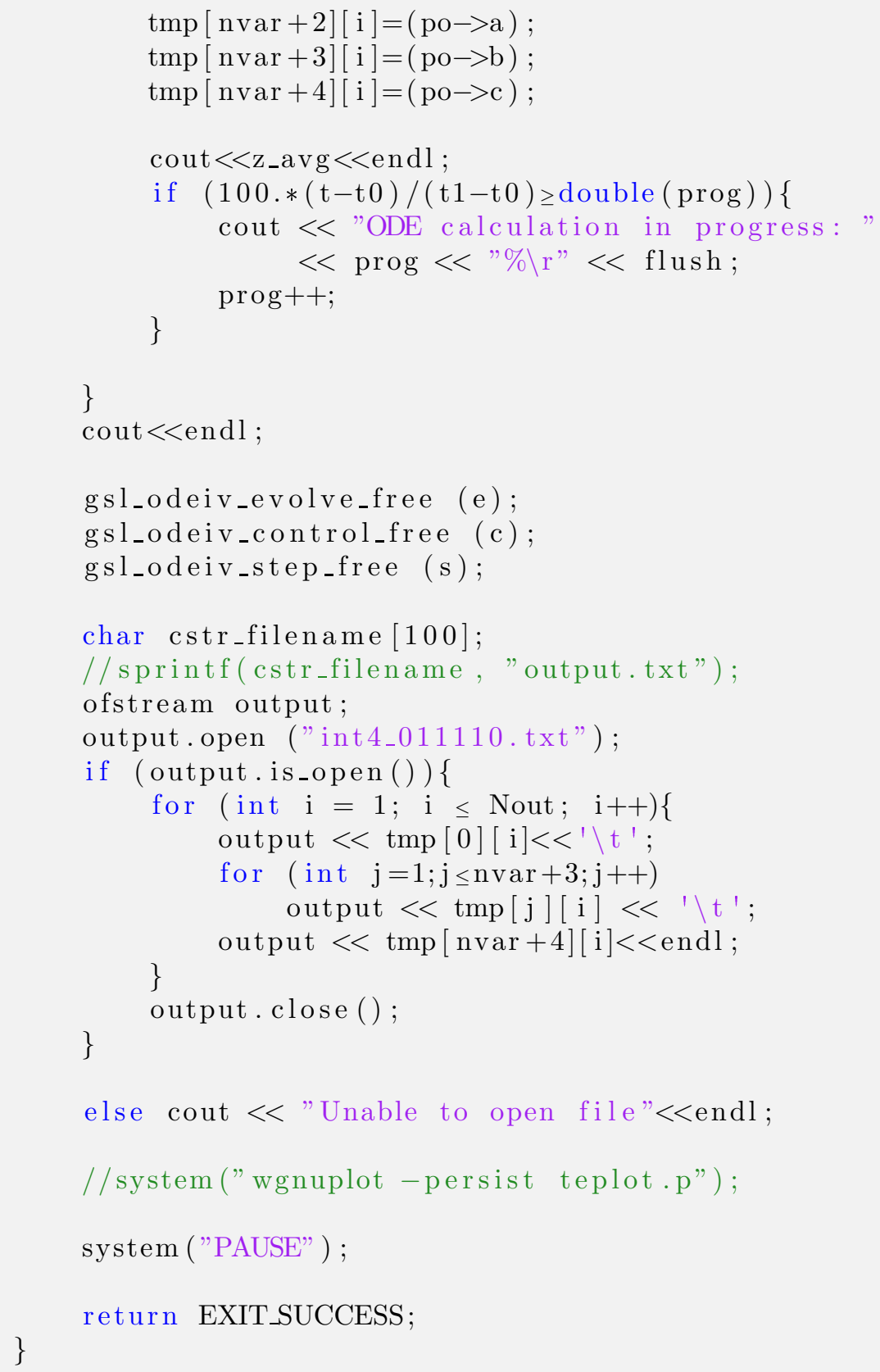


The file that defines the coupled ODEs, cluster.h, is listed here. The header files, nr3.h, expint.h, and roots.h, can be found in Ref. [125].

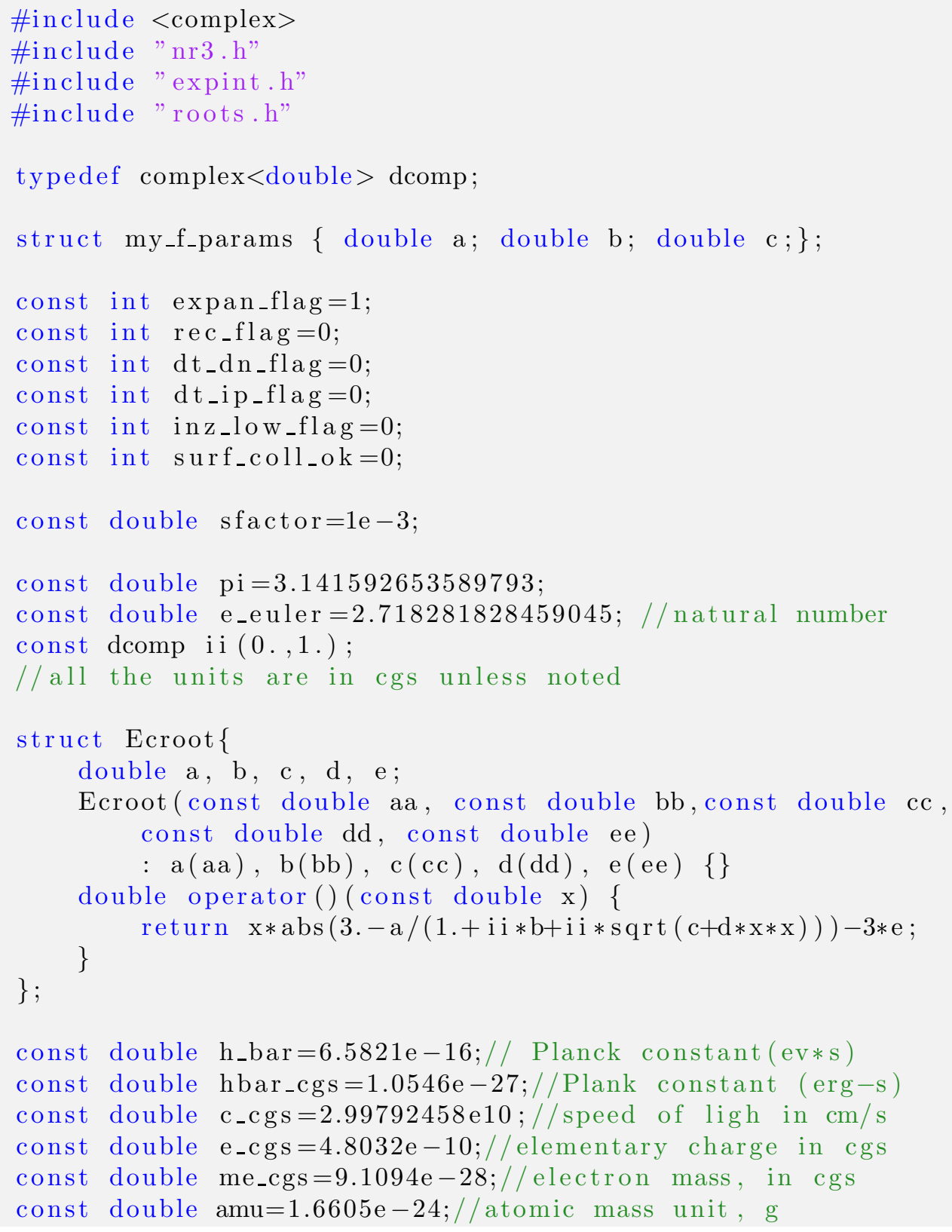




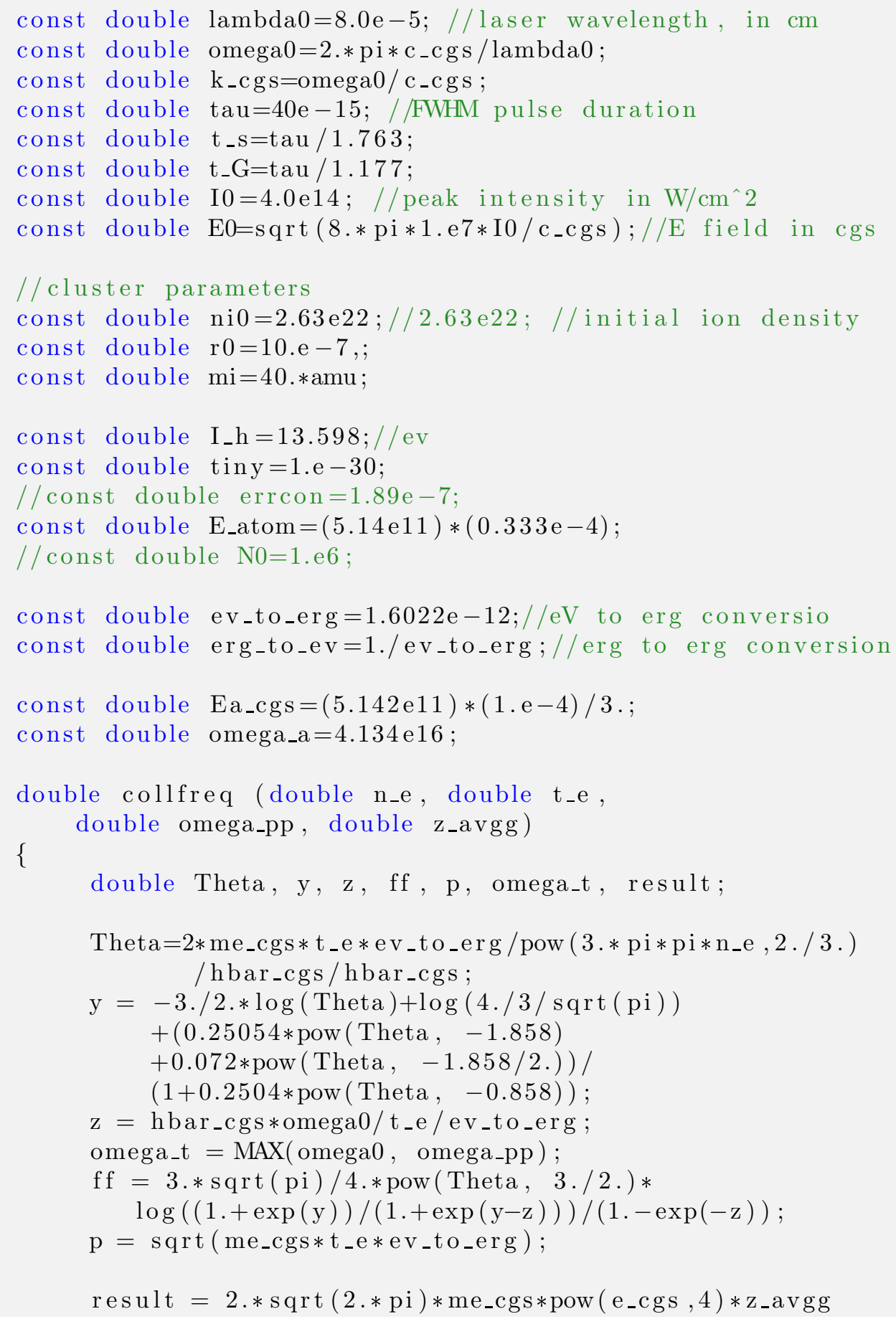




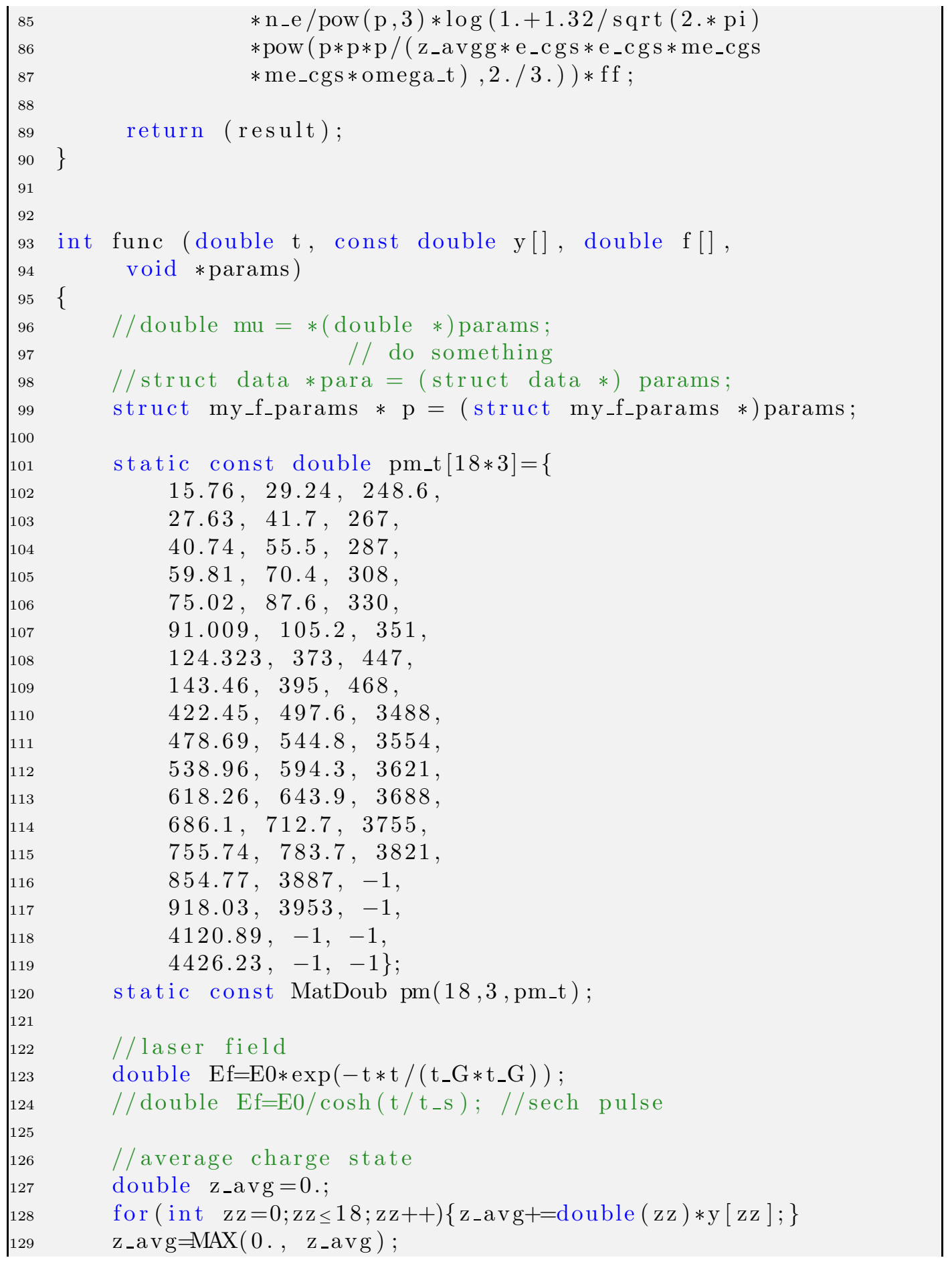




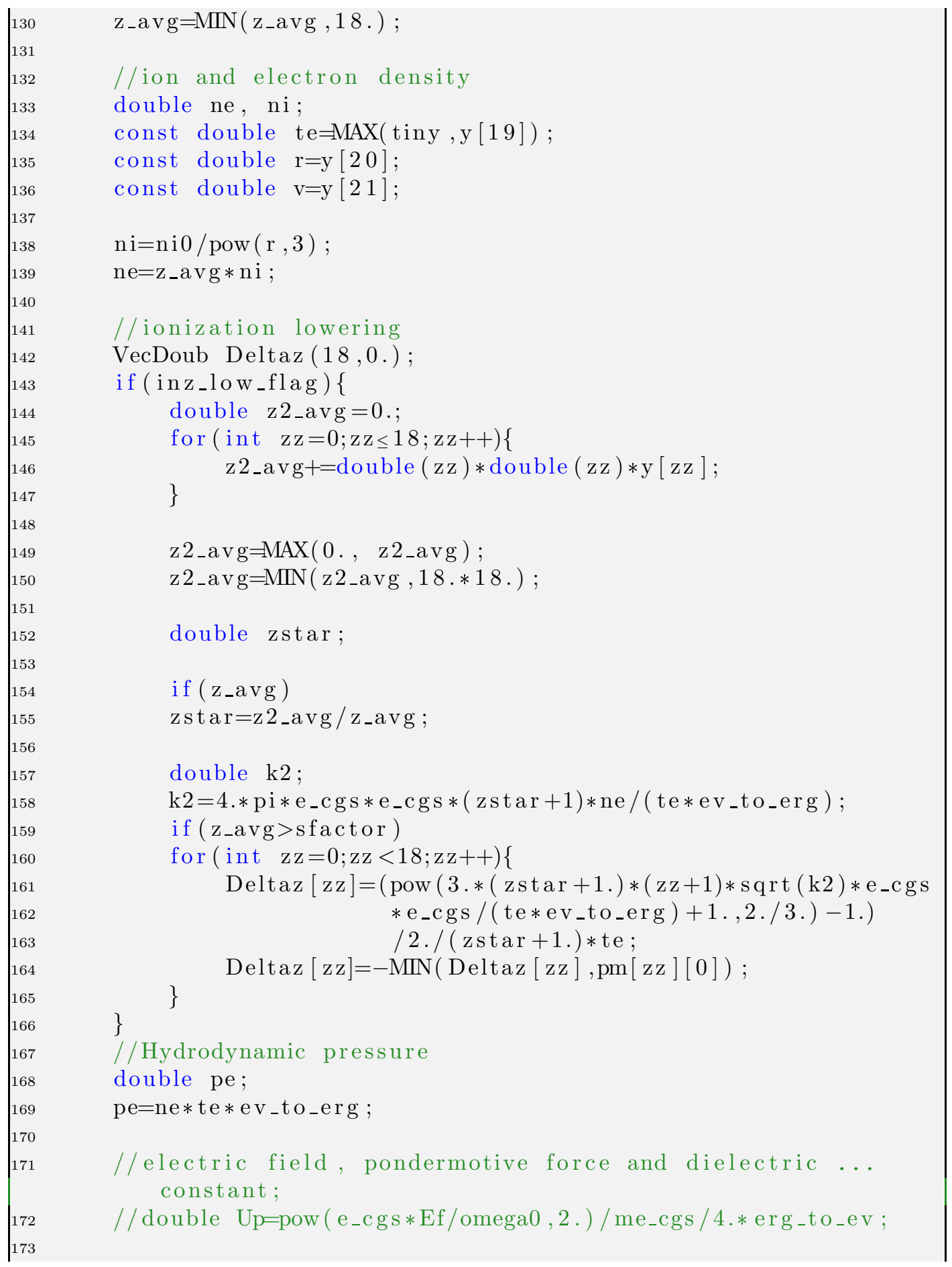




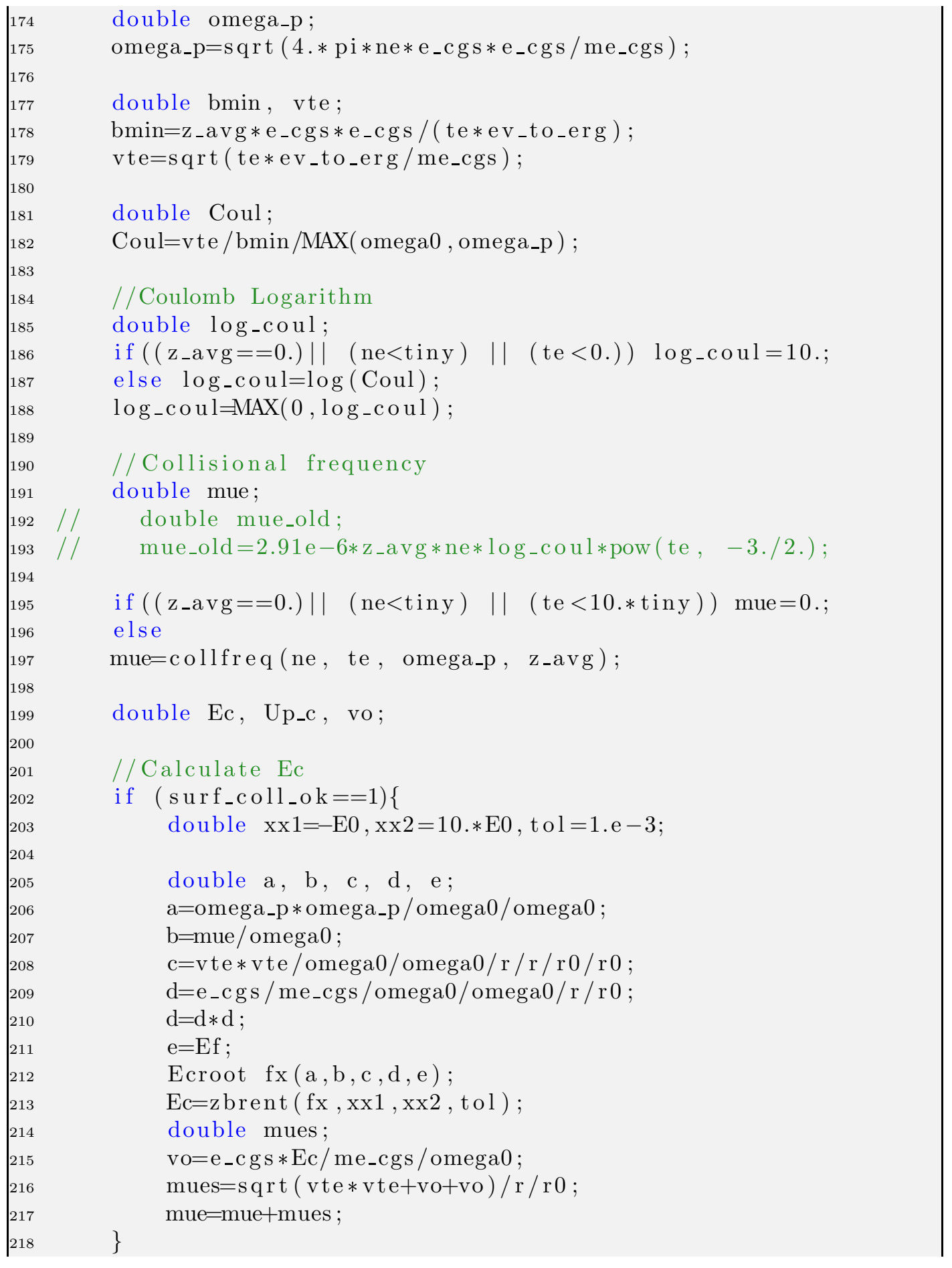




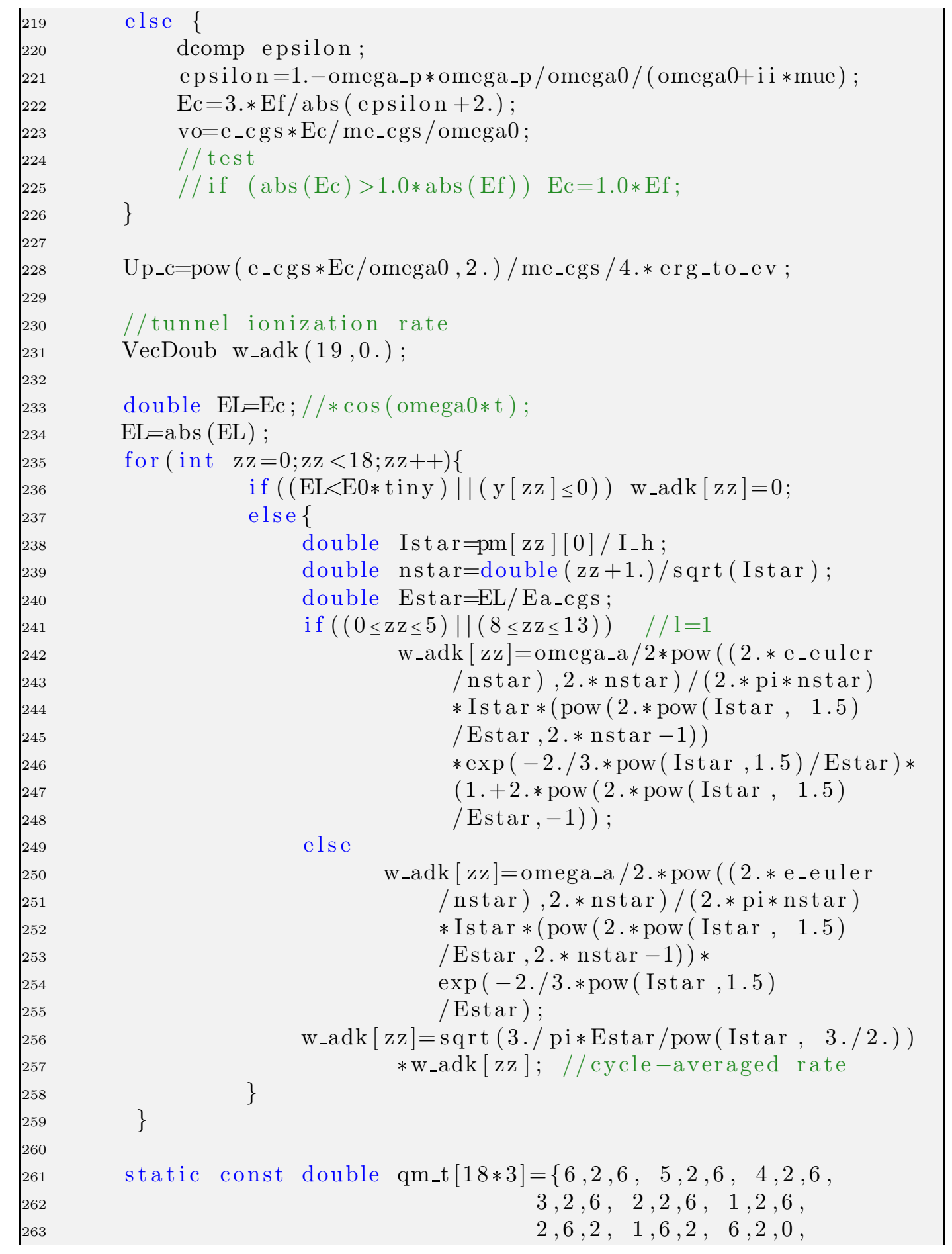




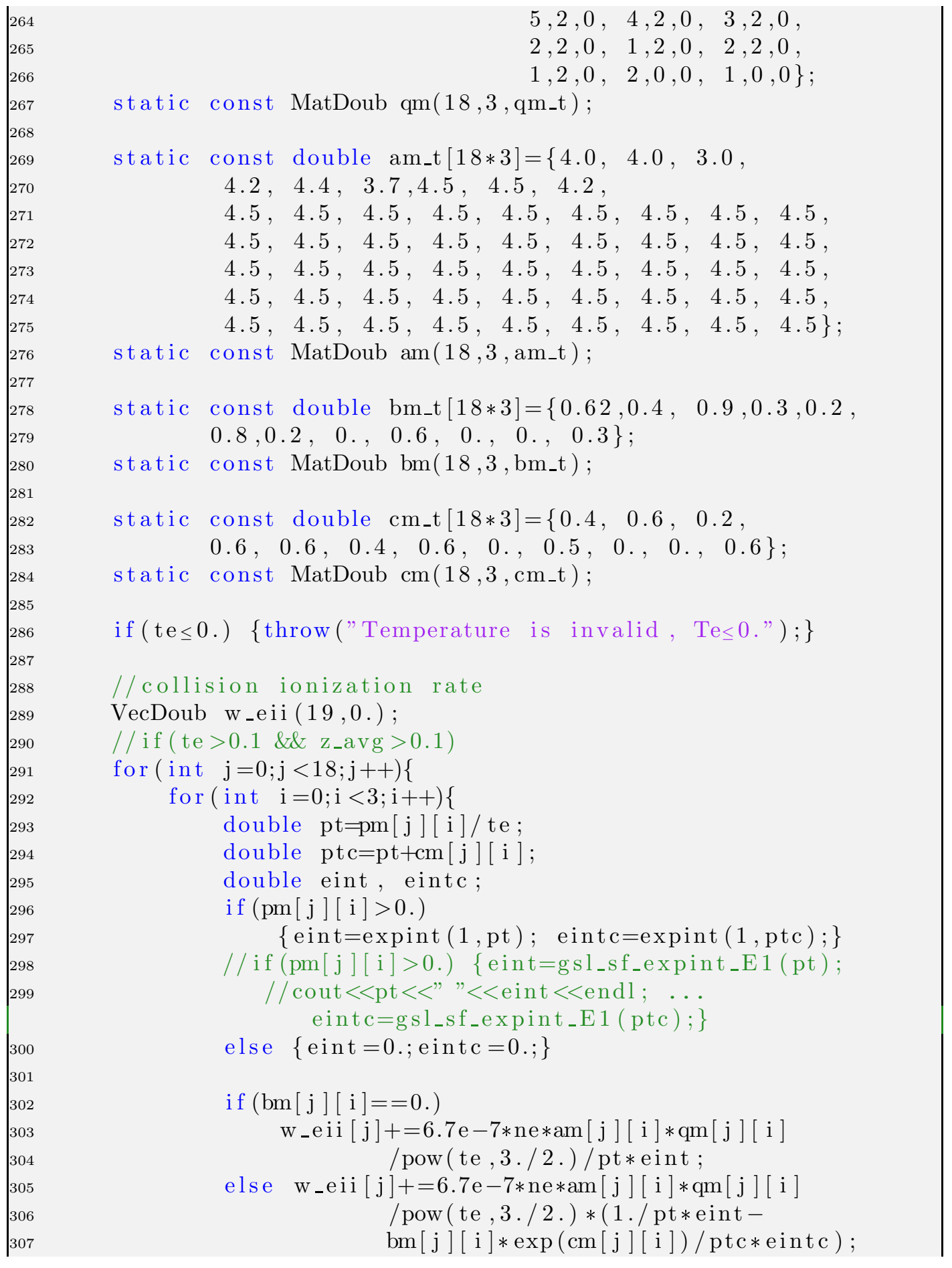




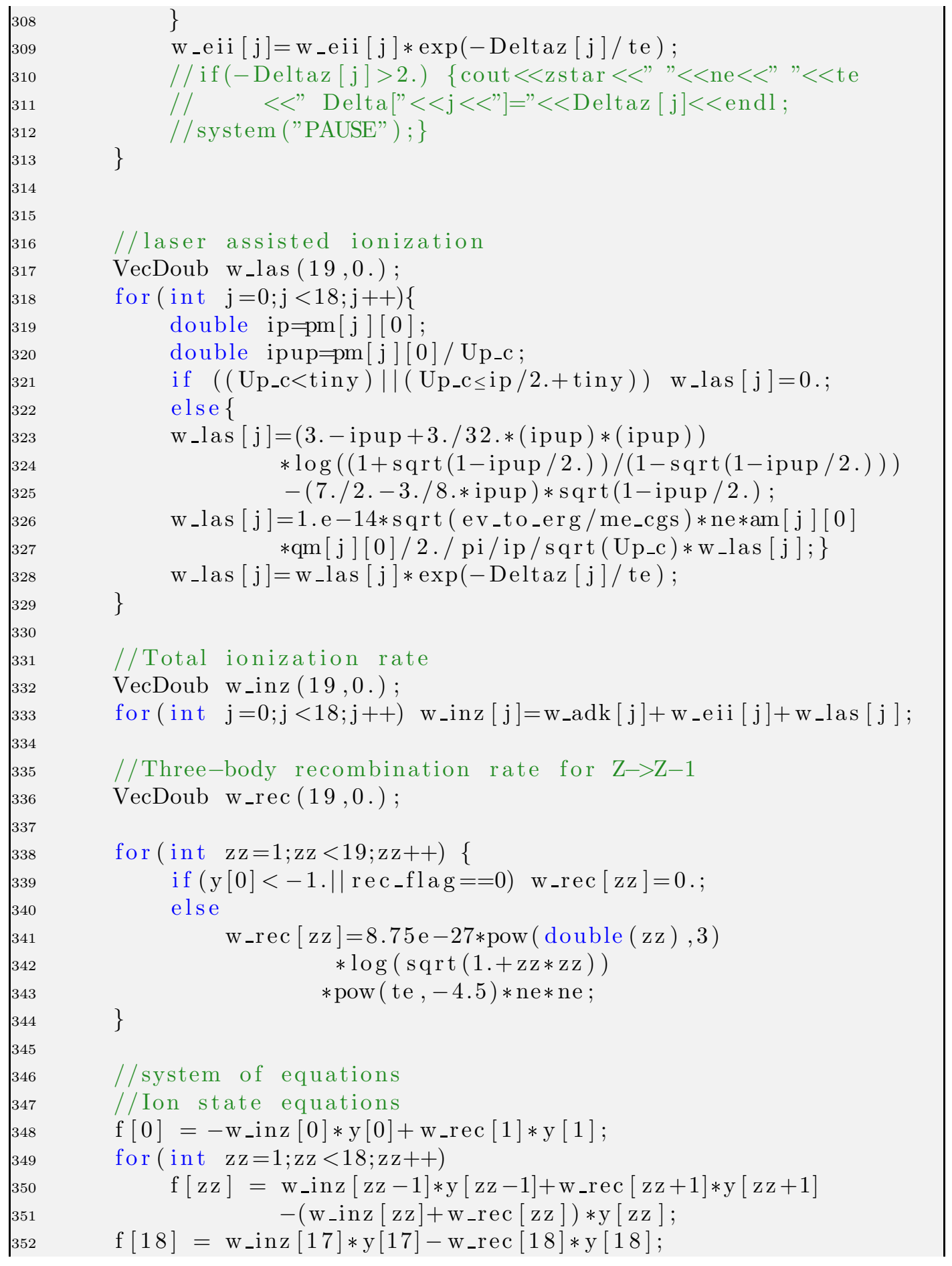




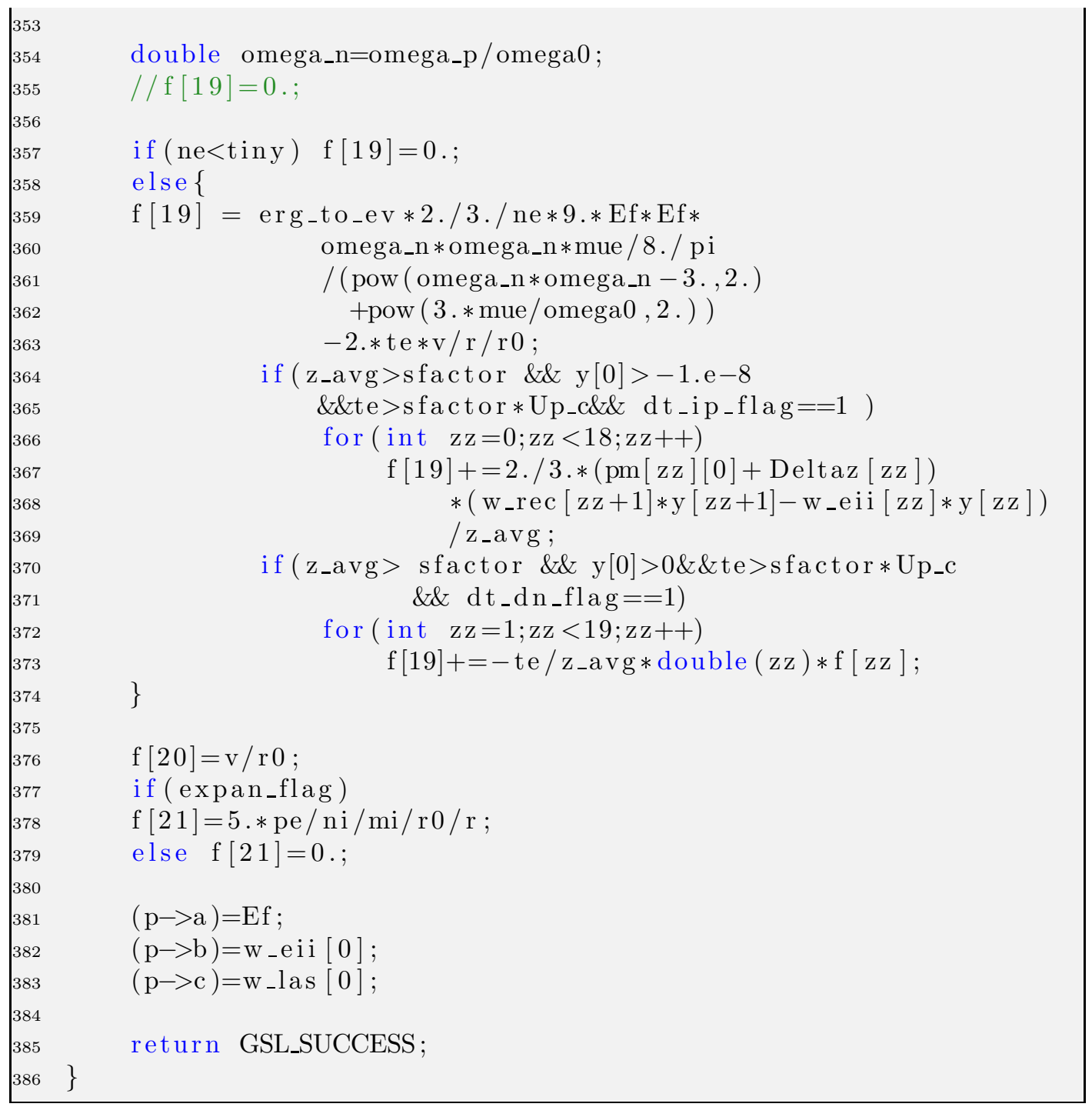




\section{Bibliography}

[1] E. W. Becker, K. Bier, and W. Henkes. Strahlen aus kondensierten atomen und molekeln im hochvakuum. Z. Physik, 146:333-338, 1956.

[2] J. Farges, M.F. de Feraudy, B. Raoult, and G. Torchet. Structure and temperature of rare gas clusters in a supersonic expansion. Surface Science, 106(1-3):95 - 100, 1981.

[3] O. F. Hagena and W. Obert. Cluster formation in expanding supersonic jets - effect of pressure, temperature, nozzle size, and test gas. J. Chem. Phys., 56(5):1793-1802, 1972.

[4] T. Fennel, K. H. Meiwes-Broer, J. Tiggesbäumker, P. G. Reinhard, P. M. Dinh, and E. Suraud. Laser-driven nonlinear cluster dynamics. Rev. Mod. Phys., 82(2):1793-1842, 2010.

[5] K. W. Madison, P. K. Patel, D. Price, A. Edens, M. Allen, T. E. Cowan, J. Zweiback, and T. Ditmire. Fusion neutron and ion emission from deuterium and deuterated methane cluster plasmas. Phys. Plasmas, 11(1):270-277, 2004.

[6] Y. Fukuda, Y. Akahane, M. Aoyama, Y. Hayashi, T. Homma, N. Inoue, M. Kando, S. Kanazawa, H. Kiriyama, S. Kondo, H. Kotaki, S. Masuda, M. Mori, A. Yamazaki, K. Yamakawa, E. Y. Echkina, I. N. Inovenkov, 
J. Koga, and S. V. Bulanov. Ultrarelativistic electron generation during the intense, ultrashort laser pulse interaction with clusters. Phys. Lett. A, 363(1-2):130-135, 2007.

[7] Lu Zhang, Li-Ming Chen, Wei-Ming Wang, Wen-Chao Yan, Da-Wei Yuan, Jing-Yi Mao, Zhao-Hua Wang, Cheng Liu, Zhong-Wei Shen, Anatoly Faenov, Tatiana Pikuz, Da-Zhang Li, Yu-Tong Li, Quan-Li Dong, Xin Lu, Jing-Long Ma, Zhi-Yi Wei, Zheng-Ming Sheng, and Jie Zhang. Electron acceleration via high contrast laser interacting with submicron clusters. Appl. Phys. Lett., 100(1):014104, 2012.

[8] Y. Fukuda, A. Y. Faenov, M. Tampo, T. A. Pikuz, T. Nakamura, M. Kando, Y. Hayashi, A. Yogo, H. Sakaki, T. Kameshima, A. S. Pirozhkov, K. Ogura, M. Mori, T. Z. Esirkepov, J. Koga, A. S. Boldarev, V. A. Gasilov, A. I. Magunov, T. Yamauchi, R. Kodama, P. R. Bolton, Y. Kato, T. Tajima, H. Daido, and S. V. Bulanov. Energy increase in multi-mev ion acceleration in the interaction of a short pulse laser with a cluster-gas target. Phys. Rev. Lett., 103(16):165002, 2009.

[9] V. Kumarappan, K. Y. Kim, and H. M. Milchberg. Guiding of intense laser pulses in plasma waveguides produced from efficient, femtosecond end-pumped heating of clustered gases. Phys. Rev. Lett., 94(20):205004, 2005.

[10] T. D. Donnelly, T. Ditmire, K. Neuman, M. D. Perry, and R. W. Falcone. High-order harmonic generation in atom clusters. Phys. Rev. Lett., 
76(14):2472-2475, 1996.

[11] F. Dorchies, F. Blasco, C. Bonté, T. Caillaud, C. Fourment, and O. Peyrusse. Observation of subpicosecond x-ray emission from laser-cluster interaction. Phys. Rev. Lett., 100(20):205002, 2008.

[12] P. M. Paul, E. S. Toma, P. Breger, G. Mullot, F. Augé, P. Balcou, H. G. Muller, and P. Agostini. Observation of a train of attosecond pulses from high harmonic generation. Science, 292(5522):1689-1692, 2001.

[13] H. C. Kapteyn, M. M. Murnane, and I. R. Christov. Extreme nonlinear optics: A coherent x rays from lasers. Physics Today, 58(3):39-44, 2005.

[14] Shambhu Ghimire, Georges Ndabashimiye, and David A. Reis. Highorder harmonic generation in solid argon. In CLEO: QELS-Fundamental Science, page QW1F.1. Optical Society of America, 2012.

[15] C. Thaury, F. Quéré, J.-P. Geindre, A. Levy, T. Ceccotti, P. Monot, M. Bougeard, F. Réau, P. d'Oliveira, P. Audebert, R. Marjoribanks, and Ph. Martin. Plasma mirrors for ultrahigh-intensity optics. Nat. Phys., 3(6):424-429, 2007.

[16] S. Gordienko, A. Pukhov, O. Shorokhov, and T. Baeva. Relativistic doppler effect: Universal spectra and zeptosecond pulses. Phys. Rev. Lett., 93:115002, 2004. 
[17] M. Lewenstein, P. Balcou, M. Y. Ivanov, A. L'Huillier, and P. B. Corkum. Theory of high-harmonic generation by low-frequency laser fields. Phys. Rev. A, 49(3):2117-2132, 1994.

[18] P. B. Corkum. Plasma perspective on strong-field multiphoton ionization. Phys. Rev. Lett., 71(13):1994-1997, 1993.

[19] Jeffrey L. Krause, Kenneth J. Schafer, and Kenneth C. Kulander. Highorder harmonic generation from atoms and ions in the high intensity regime. Phys. Rev. Lett., 68:3535-3538, 1992.

[20] Xiaoshi Zhang. Extreme Nonlinear Optics for Coherent X-ray Generation. PhD thesis, University of Colorado Boulder, 2007.

[21] G. Tempea and T. Brabec. Optimization of high-harmonic generation. Appl. Phys. B, 70:S197-S202, 2000.

[22] R. A. Ganeev, M. Suzuki, M. Baba, H. Kuroda, and T. Ozaki. Strong resonance enhancement of a single harmonic generated in the extreme ultraviolet range. Opt. Lett., 31(11):1699-1701, 2006.

[23] V. Strelkov. Role of autoionizing state in resonant high-order harmonic generation and attosecond pulse production. Phys. Rev. Lett., 104(12):123901, 2010.

[24] R. Kopold, W. Becker, and M. Kleber. Model calculations of highharmonic generation in molecular ions. Phys. Rev. A, 58(5):4022-4038, 1998. 
[25] R. Velotta, N. Hay, M. B. Mason, M. Castillejo, and J. P. Marangos. High-order harmonic generation in aligned molecules. Phys. Rev. Lett., 87(18):183901, 2001.

[26] C. Vozzi, M. Nisoli, J. P. Caumes, G. Sansone, S. Stagira, S. De Silvestri, M. Vecchiocattivi, D. Bassi, M. Pascolini, L. Poletto, P. Villoresi, and G. Tondello. Cluster effects in high-order harmonics generated by ultrashort light pulses. Appl. Phys. Lett., 86(11):111121, 2005.

[27] D. F. Zaretsky, P. Korneev, and W. Becker. High-order harmonic generation in clusters irradiated by an infrared laser field of moderate intensity. J. Phys. B: At. Mol. Opt. Phys., 43(10):105402, 2010.

[28] M. V. Fomyts'kyi, B. N. Breizman, A. V. Arefiev, and C. Chiu. Harmonic generation in clusters. Phys. Plasmas, 11(7):3349-3359, 2004.

[29] B. Shim, G. Hays, R. Zgadzaj, T. Ditmire, and M. C. Downer. Enhanced harmonic generation from expanding clusters. Phys. Rev. Lett., 98(12):123902, 2007.

[30] M. Kundu, S. V. Popruzhenko, and D. Bauer. Harmonic generation from laser-irradiated clusters. Phys. Rev. A, 76(3):033201, 2007.

[31] S. V. Popruzhenko, M. Kundu, D. F. Zaretsky, and D. Bauer. Harmonic emission from cluster nanoplasmas subject to intense short laser pulses. Phys. Rev. A, 77(6):063201, 2008. 
[32] F. Quere, C. Thaury, P. Monot, S. Dobosz, P. Martin, J. P. Geindre, and P. Audebert. Coherent wake emission of high-order harmonics from overdense plasmas. Phys. Rev. Lett., 96:125004, 2006.

[33] S. V. Bulanov, N. M. Naumova, and F. Pegoraro. Interaction of an ultrashort, relativistically strong laser pulse with an overdense plasma. Phys. Plasmas, 1(3):745-757, 1994.

[34] J. Tate, T. Auguste, H. G. Muller, P. Salières, P. Agostini, and L. F. DiMauro. Scaling of wave-packet dynamics in an intense midinfrared field. Phys. Rev. Lett., 98:013901, 2007.

[35] L. E. Chipperfield, J. S. Robinson, J. W. G. Tisch, and J. P. Marangos. Ideal waveform to generate the maximum possible electron recollision energy for any given oscillation period. Phys. Rev. Lett., 102(6):063003, 2009.

[36] E. Constant, D. Garzella, P. Breger, E. Mevel, C. Dorrer, C. Le Blanc, F. Salin, and P. Agostini. Optimizing high harmonic generation in absorbing gases: Model and experiment. Phys. Rev. Lett., 82(8):16681671, 1999.

[37] Pascal Salières and Ivan Christov. Macroscopic effects in high-order harmonic generation. In T. Brabec, editor, Strong Field Laser Physics. Springer, 2008. 
[38] C. G. Durfee, A. R. Rundquist, S. Backus, C. Herne, M. M. Murnane, and H. C. Kapteyn. Phase matching of high-order harmonics in hollow waveguides. Phys. Rev. Lett., 83(11):2187-2190, 1999.

[39] Emily A. Gibson, Ariel Paul, Nick Wagner, Ra'anan Tobey, David Gaudiosi, Sterling Backus, Ivan P. Christov, Andy Aquila, Eric M. Gullikson, David T. Attwood, Margaret M. Murnane, and Henry C. Kapteyn. Coherent soft x-ray generation in the water window with quasi-phase matching. Science, 302(5642):95-98, 2003.

[40] M. Zepf, B. Dromey, M. Landreman, P. Foster, and S. M. Hooker. Bright quasi-phase-matched soft-x-ray harmonic radiation from argon ions. Phys. Rev. Lett., 99:143901, 2007.

[41] T. Tajima, Y. Kishimoto, and M. C. Downer. Optical properties of cluster plasma. Phys. Plasmas, 6(10):3759-3764, 1999.

[42] T. Ditmire, T. Donnelly, A. M. Rubenchik, R. W. Falcone, and M. D. Perry. Interaction of intense laser pulses with atomic clusters. Phys. Rev. A, 53(5):3379-3402, 1996.

[43] S. Micheau, H. Jouin, and B. Pons. Modified nanoplasma model for laser-cluster interaction. Phys. Rev. A, 77(5):053201, 2008.

[44] F. Megi, M. Belkacem, M. A. Bouchene, E. Suraud, and G. Zwicknagel. On the importance of damping phenomena in clusters irradiated by in- 
tense laser fields. J. Phys. B: At. Mol. Opt. Phys., 36(2):273-282, 2003.

[45] T. Bornath, P. Hilse, and M. Schlanges. Ionization dynamics in nanometersized clusters interacting with intense laser fields. Laser Physics, 17:591603, 2007.

[46] J. Zweiback, T. Ditmire, and M. D. Perry. Femtosecond time-resolved studies of the dynamics of noble-gas cluster explosions. Phys. Rev. A, 59(5):R3166-R3169, 1999.

[47] H. M. Milchberg, S. J. McNaught, and E. Parra. Plasma hydrodynamics of the intense laser-cluster interaction. Phys. Rev. E, 64(5):056402, 2001.

[48] K. Y. Kim, I. Alexeev, E. Parra, and H. M. Milchberg. Time-resolved explosion of intense-laser-heated clusters. Phys. Rev. Lett., 90(2):023401, 2003.

[49] A. Gupta, T. M. Antonsen, and H. M. Milchberg. Propagation of intense short laser pulses in a gas of atomic clusters. Phys. Rev. E, 70(4):046410, 2004.

[50] A. V. Arefiev, X. Gao, M. R. Tushentsov, X. Wang, B Shim, B. N. Breizman, and M. C. Downer. Size distribution and mass fraction of microclusters in laser-irradiated plasmas. High Energy Density Phys., 6:121-127, 2010. 
[51] A. Arefiev, B. Breizman, V. Khudik, X. Gao, and M. Downer. Generation of fast ions by microclusters. Plasma Fusion Res., 5:S2071, 2010.

[52] A. V. Arefiev. private communication, 2010.

[53] M. V. Ammosov, N. B. Delone, and V. P. Krainov. Tunnel ionization of complex atoms and of atomic ions in an alternating electromagnetic field. Sov. Phys. JETP, 64:1191, 1986.

[54] B. Walker, B. Sheehy, L. F. Dimauro, P. Agostini, K. J. Schafer, and K. C. Kulander. Precision-measurement of strong-field double-ionization of helium. Phys. Rev. Lett., 73(9):1227-1230, 1994.

[55] Armin Scrinzi, Michael Geissler, and Thomas Brabec. Ionization above the coulomb barrier. Phys. Rev. Lett., 83:706-709, 1999.

[56] J. D. Jackson. Classical Electrodynamics, 3rd Edition. Wiley, 1998.

[57] W. Lotz. Electron-impact ionization cross-sections and ionization rate coefficients for atoms and ions from hydrogen to calcium. Zeitschrift fr Physik A Hadrons and Nuclei, 216:241-247, 1968.

[58] P. Hilse, M. Moll, M. Schlanges, and T. Bornath. Laser-cluster-interaction in a nanoplasma-model with inclusion of lowered ionization energies. Laser Phys., 19(3):428-436, 2009.

[59] J. C. Stewart and K. D. Pyatt, Jr. Lowering of ionization potentials in plasmas. Astrophys. J., 144:1203, 1966. 
[60] J. Zweiback. Resonance Effects in Laser Cluster Interactions. PhD thesis, University of California, Davis, 1999.

[61] A. V. Gurevich and L. P. Pitaevskii. Recombination coefficient in a dense low-temperature plasma. Sov. Phys. JETP, 19:870-871, 1964.

[62] K. Eidmann, J. Meyer-ter Vehn, T. Schlegel, and S. Hüller. Hydrodynamic simulation of subpicosecond laser interaction with solid-density matter. Phys. Rev. E, 62:1202-1214, 2000.

[63] J. Meyer-ter Vehn, A. Tronnier, and Yu Cang. Physical collision frequency $\nu_{\text {eff }}(t, \omega)$ for metals and warm dense matter. 35th EPS Conference on Plasma Phys. Hersonissos, 9-13 June 2008 ECA, 32D:P2.132, 2008.

[64] R. Ramis, K. Eidmann, J. Meyer-ter Vehn, and S. Hueller. Multi-fs - a computer code for laser-plasma interaction in the femtosecond regime. Computer Physics Communications, 183(3):637-655, 2012.

[65] S. Atzeni and J. Meyer-ter Vehn. The Physics of Inertial Fusion: Beam Plasma Interaction, Hydrodynamics, Hot Dense Matter. International Series of Monographs on Physics. Oxford University Press, USA, 2004.

[66] O. G. Danylchenko, Y. S. Doronin, S. I. Kovalenko, and V. N. Samovarov. Phase separation into pure components in mixed Ar-Xe clusters. JETP Lett., 84:324-328, 2006. 
[67] B. W. van de Waal, G. Torchet, and M. F. de Feraudy. Structure of large argon clusters $\operatorname{Ar}_{N}, 10^{3}<N<10^{5}$ : experiments and simulations. Chem. Phys. Lett., 331(1):57-63, 2000.

[68] C. Rose-Petruck, K. J. Schafer, K. R. Wilson, and C. P. J. Barty. Ultrafast electron dynamics and inner-shell ionization in laser driven clusters. Phys. Rev. A, 55(2):1182-1190, 1997.

[69] B. Breizman and A. Arefiev. Electron response in laser-irradiated microclusters. Plasma Physics Reports, 29:593-597, 2003.

[70] J. Abdallah, A. Y. Faenov, I. Y. Skobelev, A. I. Magunov, T. A. Pikuz, T. Auguste, P. D'Oliveira, S. Hulin, and P. Monot. Hot-electron influence on the x-ray emission spectra of Ar clusters heated by a highintensity 60-fs laser pulse. Phys. Rev. A, 63(3):032706, 2001.

[71] M. E. Sherrill, Jr. Abdallah, J., G. Csanak, E. S. Dodd, A. Ya. Faenov, A. I. Magunov, T. A. Pikuz, and I. Yu. Skobelev. Spectroscopic characterization of an ultrashort-pulse-laser-driven Ar cluster target incorporating both Boltzmann and particle-in-cell models. Phys. Rev. E, 73(6):066404, 2006.

[72] A. B. Langdon. Non-linear inverse bremsstrahlung and heated-electron distributions. Phys. Rev. Lett., 44(9):575-579, 1980.

[73] M. Krishnamurthy, J. Jha, D. Mathur, C. Jungreuthmayer, L. Ramunno, J. Zanghellini, and T. Brabec. Ion charge state distribution in the laser- 
induced coulomb explosion of argon clusters. J. Phys. B: At. Mol. Opt. Phys., 39(3):625-632, 2006.

[74] J. Jha and M. Krishnamurthy. Hotter electron generation in doped clusters. J. Phys. B: At. Mol. Opt. Phys., 41(4):041002, 2008.

[75] D. Miller. Free jet sources. In Giacinto Scoles, editor, Atomic and Molecular Beam Methods, volume 1. Oxford University Press, 1988.

[76] R. Karnbach, M. Joppien, J. Stapelfeldt, J. Wormer, and T. Moller. CLULU - an experimental setup for luminescence measurements on van der Waals clusters with synchrotron-radiation. Rev. Sci. Instrum., 64(10):2838-2849, 1993.

[77] O. F. Hagena. Cluster ion sources. Rev. Sci. Instrum., 63(4):23742379, 1992.

[78] Yunquan Liu, Quanli Dong, Xiaoyu Peng, Zan Jin, and Jie Zhang. Soft x-ray emission, angular distribution of hot electrons, and absorption studies of argon clusters in intense laser pulses. Phys. Plasmas, 16(4):043301, 2009.

[79] U. Buck and R. Krohne. Cluster size determination from diffractive he atom scattering. J. Chem. Phys., 105(13):5408-5415, 1996.

[80] F. Dorchies, F. Blasco, T. Caillaud, J. Stevefelt, C. Stenz, A. S. Boldarev, and V. A. Gasilov. Spatial distribution of cluster size and density 
in supersonic jets as targets for intense laser pulses. Phys. Rev. A, 68(2):023201, 2003.

[81] L. A. Lompré, M. Ferray, A. L'Huillier, X. F. Li, and G. Mainfray. Optical determination of the characteristics of a pulsed-gas jet. J. Appl. Phys., 63(5):1791-1793, 1988.

[82] M. Takeda, H. Ina, and S. Kobayashi. Fourier-transform method of fringe-pattern analysis for computer-based topography and interferometry. J. Opt. Soc. Am., 72(1):156-160, 1982.

[83] M. Kalal and K. A. Nugent. Abel inversion using fast fourier-transforms. Applied Optics, 27(10):1956-1959, 1988.

[84] F. Brandi and F. Giammanco. Temporal and spatial characterization of a pulsed gas jet by a compact high-speed high-sensitivity secondharmonic interferometer. Opt. Express, 19(25):25479-25487, 2011.

[85] K. L. Saenger and J. B. Fenn. On the time required to reach fullydeveloped flow in pulsed supersonic free jets. J. Chem. Phys., 79(12):6043$6045,1983$.

[86] K. Y. Kim, V. Kumarappan, and H. M. Milchberg. Measurement of the average size and density of clusters in a gas jet. Appl. Phys. Lett., 83(15):3210-3212, 2003.

[87] D. S. Bethune. Optical second-harmonic generation in atomic vapors with focused beams. Phys. Rev. A, 23:3139-3151, 1981. 
[88] J. M. Soler, N. García, O. Echt, K. Sattler, and E. Recknagel. Microcluster growth - transition from successive monomer addition to coagulation. Phys. Rev. Lett., 49(25):1857-1860, 1982.

[89] J. Wörmer, V. Guzielski, J. Stapelfeldt, and T. Möller. Fluorescence excitation spectroscopy of xenon clusters in the vuv. Chem. Phys. Lett., 159(4):321-326, 1989.

[90] J. Farges, M. F. Deferaudy, B. Raoult, and G. Torchet. Noncrystalline structure of argon clusters .2. multilayer icosahedral structure of $\operatorname{Ar}_{N}$ clusters $50<N<750$. J. Chem. Phys., 84(6):3491-3501, 1986.

[91] E. Fort, F. Pradere, A. De Martino, H. Vach, and M. Chatelet. Diagnostics of mixed van der waals clusters. Eur. Phys. J. D, 1(1):79-84, 1998.

[92] A. Ramos, J. M. Fernandez, G. Tejeda, and S. Montero. Quantitative study of cluster growth in free-jet expansions of $\mathrm{CO}_{2}$ by Rayleigh and Raman scattering. Phys. Rev. A, 72(5):053204, 2005.

[93] Y. M. Chen, M. Y. Hsu, Y. H. Hsieh, J. Y. Lin, J. Wang, and S. Y. Chen. Enhancement of high-harmonic generation by laser-induced cluster vibration. Opt. Lett., 32(18):2714-2716, 2007.

[94] A. S. Boldarev, V. A. Gasilov, A. Y. Faenov, Y. Fukuda, and K. Yamakawa. Gas-cluster targets for femtosecond laser interaction: Modeling and optimization. Rev. Sci. Instrum., 77:083112, 2006. 
[95] M. A. Ratner. Kinetics of cluster growth in expanding rare-gas jet. Low Temp. Phys., 25(4):266-273, 1999.

[96] Christophe Prigent, Cornelia Deiss, Emily Lamour, Jean-Pierre Rozet, Dominique Vernhet, and Joachim Burgdoerfer. Effect of pulse duration on the x-ray emission from Ar clusters in intense laser fields. Phys. Rev. A, 78(5):053201, 2008.

[97] J. W. Keto, H. Cai, M. Kykta, C. Lei, T. Moller, and G. Zimmerer. Two-photon spectroscopy of xenon dimers in supersonic jets. J. Chem. Phys., 107(16):6080-6093, 1997.

[98] Y. Fukuda, K. Yamakawa, Y. Akahane, M. Aoyama, N. Inoue, H. Ueda, J. Abdallah, G. Csanak, A. Y. Faenov, A. I. Magunov, T. A. Pikuz, I. Y. Skobelev, A. S. Boldarev, and V. A. Gasilov. X-ray study of microdroplet plasma formation under the action of superintense laser radiation. JETP Lett., 78(3):115-118, 2003.

[99] Guanglong Chen, Byunghoon Kim, Byungnam Ahn, and Dong Eon Kim. Pressure dependence of argon cluster size for different nozzle geometries. J. Appl. Phys., 106(5):053507, 2009.

[100] X. Gao, X. Wang, B Shim, A. V. Arefiev, R. Korzekwa, and M. C. Downer. Characterization of cluster/monomer ratio in pulsed supersonic gas jets. Appl. Phys. Lett., 100:064101, 2012. 
[101] T. H. Keil. Polarizability of an argon atom in solid argon. J. Chem. Phys., 46(11):4404-4409, 1967.

[102] K. Y. Kim, I. Alexeev, and H. M. Milchberg. Single-shot supercontinuum spectral interferometry. Appl. Phys. Lett., 81(22):4124-4126, 2002.

[103] B. Ziaja, H. Wabnitz, E. Weckert, and T. Moeller. Atomic clusters of various sizes irradiated with short intense pulses of VUV radiation. Europhys. Lett., 82(2):24002, 2008.

[104] Wolfgang Christen, Tim Krause, Björn Kobin, and Klaus Rademann. Precision velocity measurements of pulsed supersonic jets. J. Phys. Chem. A, 115(25):6997-7004, 2011.

[105] Oommen Abraham, Jack H. Binn, Barry G. DeBoer, and Gilbert D. Stein. Gasdynamics of very small laval nozzles. Phys. Fluids, 24(6):10171031, 1981.

[106] Samuel J. E. Brockington, Robert D. Horton, David Q. Hwang, Russell W. Evans, Stephen J. Howard, and Y. C. Francis Thio. Plasma density gradient measurement using laser deflection. Rev. Sci. Instrum., 76(6):063503, 2005.

[107] A. Ya. Faenov, I. Yu. Skobelev, T. A. Pikuz, S. A. Pikuz, V. E. Fortov, Y. Fukuda, Y. Hayashi, A. Pirozhkov, H. Kotaki, T. Shimomura, H. Kiriyama, S. Kanazawa, Y. Kato, J. Colgan, J. Abdallah, and 
M. Kando. X-ray spectroscopy diagnoses of clusters surviving under prepulses of ultra-intense femtosecond laser pulse irradiation. Laser and Particle Beams, 30(03):481-488, 2012.

[108] K. J. Mendham, N. Hay, M. B. Mason, J. W. G. Tisch, and J. P. Marangos. Cluster-size distribution effects in laser-cluster interaction experiments. Phys. Rev. A, 64(5):055201, 2001.

[109] S. Backus, J. Peatross, Z. Zeek, A. Rundquist, G. Taft, M. M. Murnane, and H. C. Kapteyn. 16-fs, 1-mu j ultraviolet pulses generated by thirdharmonic conversion in air. Opt. Lett., 21(9):665-667, 1996.

[110] Y. Barad, H. Eisenberg, M. Horowitz, and Y. Silberberg. Nonlinear scanning laser microscopy by third harmonic generation. Appl. Phys. Lett., 70(8):922-924, 1997.

[111] A. B. Fedotov, A. N. Naumov, V. P. Silin, S. A. Uryupin, A. M. Zheltikov, A. P. Tarasevitch, and D. von der Linde. Third-harmonic generation in a laser-pre-excited gas: the role of excited-state neutrals. Phys. Lett. A, 271(5-6):407-412, 2000.

[112] S. Suntsov, D. Abdollahpour, D. G. Papazoglou, and S. Tzortzakis. Filamentation-induced third-harmonic generation in air via plasma-enhanced third-order susceptibility. Phys. Rev. A, 81(3):033817, 2010.

[113] Klaus Hartinger and Randy A. Bartels. Enhancement of third harmonic generation by a laser-induced plasma. Appl. Phys. Lett., 93(15):151102, 
2008.

[114] Cristina Rodriguez, Zhanliang Sun, Zhenwei Wang, and Wolfgang Rudolph. Characterization of laser-induced air plasmas by third harmonic generation. Opt. Express, 19(17):16115-16125, 2011.

[115] David Kupka and Randy A. Bartels. Optimization of third harmonic conversion efficiency in the presence of a spatially localized plasma. IEEE J. Quantum. Electron., 48(6):790-796, 2012.

[116] X. Wang, X. Gao, B. Shim, A. Arefiev, B. Breizman, and M. Downer. Anisotropic third-harmonic plasmonic resonances in expanding nanoclusters. Bulletin of the American Physical Society, 53, 2008.

[117] R. Allison, J. Burns, and A. J. Tuzzolino. Absolute fluorescent quantum efficiency of sodium salicylate. J. Opt. Soc. Am., 54(6):747-750, 1964.

[118] M. Ferray, A. L'Huillier, X. F. Li, L. A. Lompré, G. Mainfray, and C. Manus. Multiple-harmonic conversion of 1064-nm radiation in raregases. J. Phys. B: At. Mol. Opt. Phys., 21(3):L31-L35, 1988.

[119] M. Kolesik, E. M. Wright, and J. V. Moloney. Femtosecond filamentation in air and higher-order nonlinearities. Opt. Lett., 35(15):2550-2552, 2010.

[120] B Shim. Time-Resolved Study of Third Harmonic Generation from Anisotropically Expanding Clusters. PhD thesis, the University of Texas at Austin, 2006. 
[121] B. F. Murphy, K. Hoffmann, A. Belolipetski, J. Keto, and T. Ditmire. Explosion of xenon clusters driven by intense femtosecond pulses of extreme ultraviolet light. Phys. Rev. Lett., 101(20):203401, 2008.

[122] T. Laarmann, A. R. B. de Castro, P. Gurtler, W. Laasch, J. Schulz, H. Wabnitz, and T. Moller. Interaction of argon clusters with intense vuv-laser radiation: The role of electronic structure in the energydeposition process. Phys. Rev. Lett., 92(14):143401, 2004.

[123] W. F. Chan, G. Cooper, X. Guo, G. R. Burton, and C. E. Brion. Absolute optical oscillator strengths for the electronic excitation of atoms at high resolution. III. The photoabsorption of argon, krypton, and xenon. Phys. Rev. A, 46:149-171, 1992.

[124] M. Galassi, J. Davies, J. Theiler, B. Gough, G. Jungman, M. Booth, P. Alken, and F. Rossi. GNU Scientific Library Reference Manual Third Edition. Network Theory Ltd., 3rd edition, 2009.

[125] William H. Press, Saul A. Teukolsky, William T. Vetterling, and Brian P. Flannery. Numerical Recipes 3rd Edition: The Art of Scientific Computing. Cambridge University Press, New York, NY, USA, 2007. 